\title{
ENVIRONMENTAL ENRICHMENT INCREASES RESPONSE STRENGTH AND PAIRED-PULSE DEPRESSION OF AUDITORY CORTEX NEURONS
}

CHERIE R. PERCACCIO, M.S, M.S.

\author{
DISSERTATION \\ Presented to the Faculty of \\ The University of Texas at Dallas \\ in Partial Fulfillment \\ of the Requirements \\ for the Degree of
}

DOCTOR OF PHILOSOPHY IN COGNITION AND NEUROSCIENCE

THE UNIVERSITY OF TEXAS AT DALLAS

May, 2006 
UMI Number: 3210677

\section{UMI}

UMI Microform 3210677

Copyright 2006 by ProQuest Information and Learning Company.

All rights reserved. This microform edition is protected against unauthorized copying under Title 17, United States Code.

ProQuest Information and Learning Company 300 North Zeeb Road

P.O. Box 1346

Ann Arbor, MI 48106-1346 
Copyright 2006

Cherie R. Percaccio

All Rights Reserved 
ENVIRONMENTAL ENRICHMENT INCREASES RESPONSE STRENGTH AND PAIRED-PULSE DEPRESSION OF AUDITORY CORTEX NEURONS

APPROVED BY SUPERVISORY COMMITTEE:
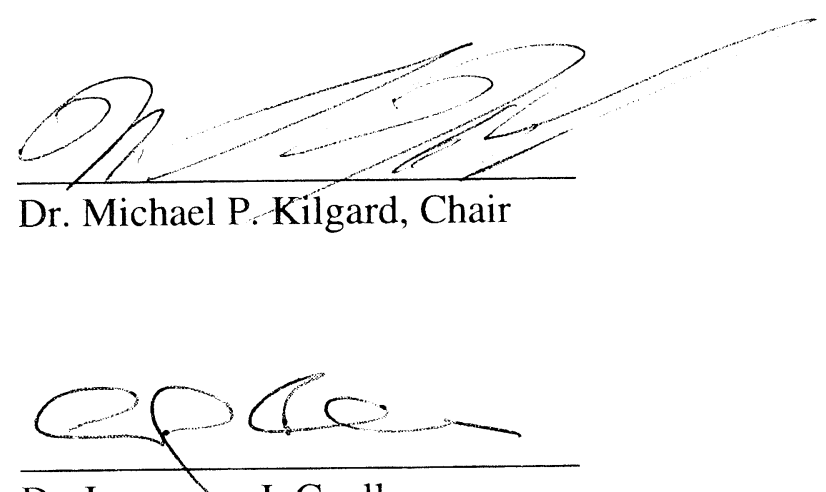

Dr. Lawrence J. Cauller

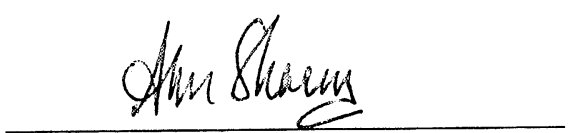

Dr. Anu Sharma

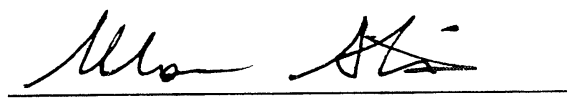

Dr. Marco Atzori 
To my family for enriching my life 


\section{PREFACE}

Abnormal responses to sensory stimuli interfere with the brain's ability to develop and learn properly. Sensory processing disorders are common, for example, among individuals with autism, dyslexia, language impairment, attention deficit disorder, and schizophrenia. Learning how to harness the brain's ability to remodel sensory networks is therefore a critical part of helping these individuals recover lost skills and develop new ones.

This dissertation contains a treasure trov of information about how rapidly the brain responds to an enriched environment as well as to the devastating effects that result from a lack of stimulation. There are intriguing insights into the relative benefits of physical activity, sensory stimulation and discovery, as well as cautionary tales concerning the effects of sensory experiences that are devoid of meaning. Clinicians have much to learn from this.

Brains adapt rapidly and robustly to the changing needs of their environments. This adaptation is reflected in the continual remodeling of sensory networks and in the behavioral results of learning. While the complex neurochemistry of learning remains poorly understood, this dissertation suggests that the act of learning depends on the ability to attach meaning and significance to sensory experiences. We can dramatically improve our ability to treat autism, dyslexia, language impairment, and attention deficit disorder by focusing our efforts on ways to help these individuals understand the meaning of their experiences.

Dr. Michelle L. MacAlpine 


\section{ACKNOWLEDGEMENTS}

I have been fortunate in my academic and professional development to associate myself with brilliant pioneers in their fields. My clinical mentor, Dr. Michelle MacAlpine, developed a play approach to treat children with autistic spectrum disorders. I literally needed to understand why the therapy was associated with a decrease in the severity of autistic symptoms, and since autism may be a brain-based disorder, I contacted a neuroscience professor. I had no idea what I was getting into, but recognized the extremely good fortune of belonging to a lab on the cutting edge of auditory neuroscience and cortical plasticity.

My intent was to mold my interest and background in autism into a series of neuroscience experiments, but I had a lot of catching up to do. One of the most important factors contributing to my success in graduate school was my advisor, Dr. Mike Kilgard. Dr. Kilgard has been an ideal mentor--advising me from my first tentative steps as a scientist to publishing papers. Probably his greatest gift to me was to recognize me as unique and to mentor me accordingly. Dr. Kilgard was a constant source of guidance, advice, constructive criticism, and positive encouragement. Several times in the process, I lost my momentum and self-confidence, but he was always there to encourage me, to tell me I am normal, and, at times, to refuse to let me quit. Now, I must agree that few experiences have been as fulfilling. I sincerely hope that Dr. Kilgard is unreservedly proud of me and believes that I reflect well on him now and will continue to do so in the future. Thank you for having faith in a psychology student--my diversified background is probably my best asset. 
Someone very wise told me that someone even wiser told him that science is not done in a vacuum... My most sincere appreciation:

To my rats: I remember every single one of you.

To past and present Ph.D. students who helped me through this process: Karen Pawlowski, Navzer Engineer, Pritesh Pandya, Raluca Moucha, Amanda Puckett, and Crystal Novitski--thank you for making graduate school seem worthwhile and for sharing my intellectual enthusiasm. Thank you to the Kilgard Lab for being my friends, sources of feedback and support, for sharing good and bad experiences with me, and for providing valuable encouragement.

To my committee members, Drs. Mike Kilgard, Lawrence Cauller, Anu Sharma, Steve Lomber, and Marco Atzori for their guidance and patience while completing this dissertation. I am the beneficiary of their expertise, and all have generously given their time and professional assistance. Thank you for allowing me to push the boundaries.

To the Graduate Studies Committee, including the Dean, Bert Moore, and the Assistant Dean, Bob Stillman, thank you for the travel awards that made it possible to present at ARO 2002-2004. Thank you also for assigning appropriate teaching assignments. These experiences were invaluable to my professional development.

To the administrative staff of the School of Behavioral and Brain Science, including Bonnie Dougherty, Abbie Bailey, Jo Valcik, and Susie Milligan—-thank you for enabling me to concentrate on science. 
To the Jones Lab at UT: thank you for providing the motivation and the remaining intellectual knowledge and equipment to complete this dissertation. I look forward to sharing the next stage in my career with ya'll--WHOOP!

To my personal cheerleaders: My husband, John—-thank you for patiently supporting me (financially and emotionally) all of these years and for being as committed to my goals as I am...thank you for always loving my drive_even when "we" are slightly irritating. When we met, I said, "Someday you will call me doctor"...I never lie. My entire family—thank you for supporting me for 28 years, and especially for the past 19. I could not have accomplished anything without your love. Trina Caudill, my very best friend, thank you for believing that everything I say about science makes sense, and for being the most encouraging person I know. To my running partner, Amanda Santeford, thank you for teaching me the importance of a slow steady pace the entire distance. I finally realized that earning a Ph.D. is similar to running a marathon.

It is acceptable for this dissertation to include authentic copies of published papers, provided they conform to UTD formatting requirements. It must include a comprehensive abstract, a full introduction, a literature review, and a final overall conclusion. The acknowledgements section at the beginning of each manuscript contains an explicit statement describing the contribution of all of the authors. The signatures of the supervising committee which precede all other material in the dissertation attest to the accuracy of this statement. The logic that connects the chapters is found in the discussion section of the preceding chapter. Enjoy the story!

May 2006 


\section{ENVIRONMENTAL ENRICHMENT INCREASES RESPONSE STRENGTH AND PAIRED-PULSE DEPRESSION OF AUDITORY CORTEX NEURONS}

Publication No.

Cherie R. Percaccio, Ph.D.

The University of Texas at Dallas, 2006

Supervising Professor: $\quad$ Michael P. Kilgard, Ph.D.

The anatomical and neurochemical consequences of enrichment on information processing have not been studied in auditory cortex. Understanding the direction of the physiologic plasticity associated with enrichment will facilitate the development of therapies tailored to specific clinical populations with auditory processing disorders. The experiments within this dissertation provide the first evidence of evoked potential plasticity in auditory cortex with an enrichment paradigm, and describe nine significant findings about the influence of housing conditions on awake rat auditory cortical evoked potentials. In chapters two and three, we recorded responses to repeated tones and noise bursts to document the time-course of changes in response strength and paired-pulse depression. In chapter four, rats were housed in conditions designed to isolate several environmental factors that may have contributed to the expression of enrichment-induced plasticity. Other rats received 
immunotoxic cholinergic lesions to determine if enrichment-induced plasticity is modulated by acetylcholine input from nucleus basalis. Collectively, the conclusions of this dissertation are: (1) Enrichment increases noise and tone-evoked response strength; however, (2) greater plasticity effects can be observed with narrowband stimuli that do not elicit saturated responses. (3) Housing condition increased or decreased cortical responses in less than 2 weeks in young and adult rats. (4) There was no difference in the scale of plasticity between adolescent and sexually mature rats. (5) Paired-pulse depression also increased or decreased depending on current housing condition (6) Enrichment also increased response strength and forward masking in rats with substantial cholinergic damage. (7) Rats that could hear the enriched environment, but not interact with it, exhibited increased tone-evoked responses and paired-pulse depression. (8) Social housing and exercise experienced in isolation had no effect on response strength. (9) Rats housed in a social environment had more paired-pulse depression than rats housed with a running wheel, but were not different than rats passively exposed to the sounds associated with the enriched environment. These results indicate that sensory experience modulates response strength and forward masking in the auditory cortex along a continuum of environmental experiences. The implications of these results are relevant to several clinical disorders, including autism, schizophrenia, and dyslexia. 


\section{TABLE OF CONTENTS}

Dedication..................................................................

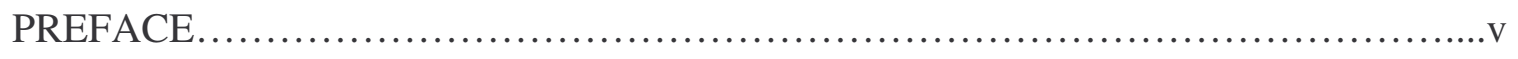

ACKNOWLEDGEMENTS ................................................................................. vi

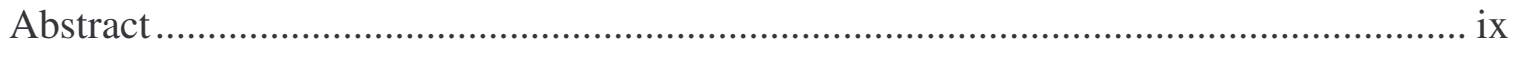

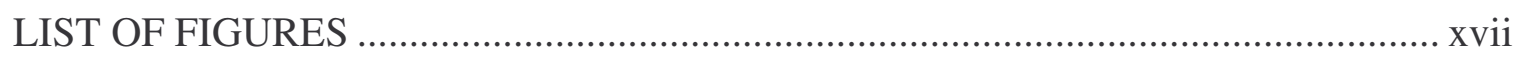

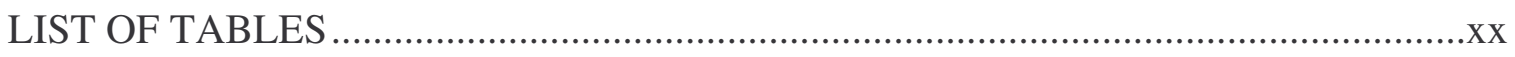

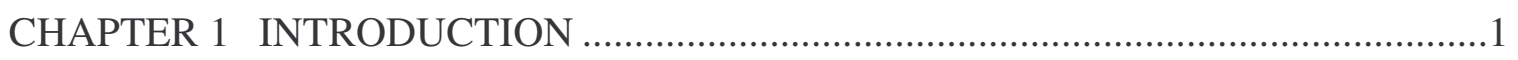

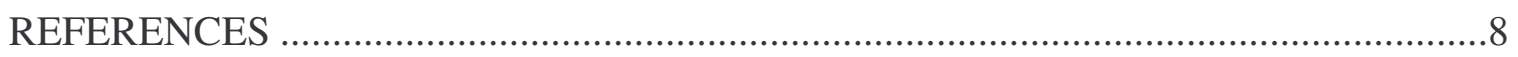

CHAPTER 2 ENVIRONMENTAL ENRICHMENT IMPROVES RESPONSE

STRENGTH, THRESHOLD, SELECTIVITY, LATENCY OF AUDITORY CORTEX

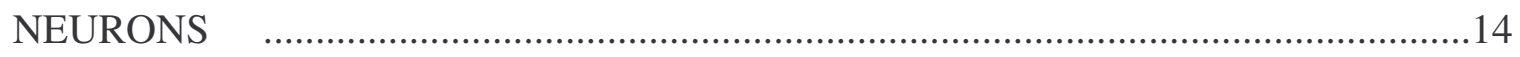

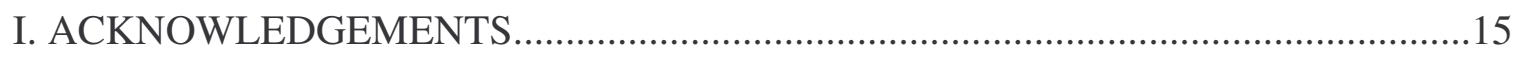

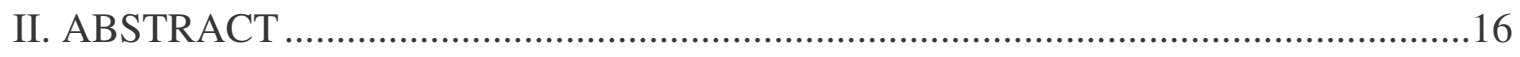

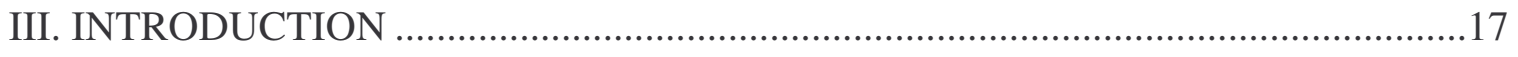

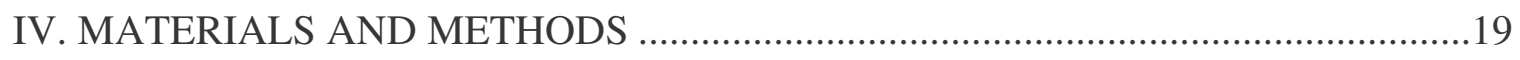

Environmental Conditions .................................................................... 19 
Experiment 1 - Extracellular Recordings .........................................................22

Acute Surgery..........................................................................23

Stimulus Presentation and Data Analysis.............................................24

Experiment 2 - Auditory Evoked Potential .....................................................225

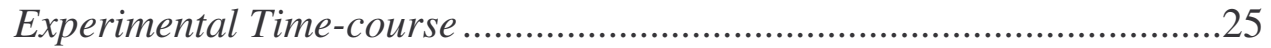

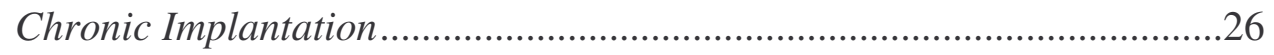

Stimulus Presentation and Data Analysis...........................................26

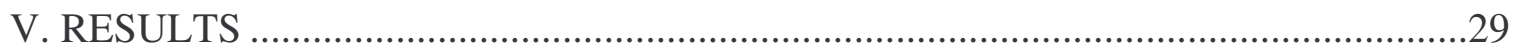

Experiment 1 - Extracellular Recordings .....................................................22

Experiment 2 - Auditory Evoked Potential .................................................33

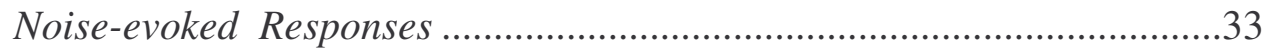

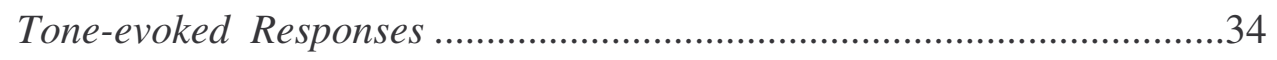

Time-course of Auditory-evoked Plasticity ............................................38

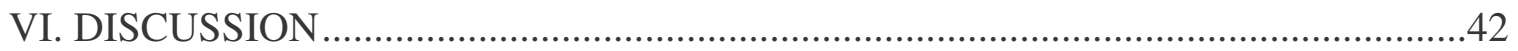

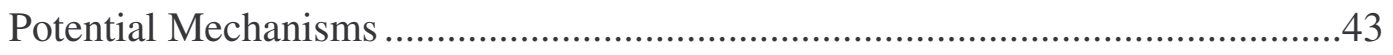

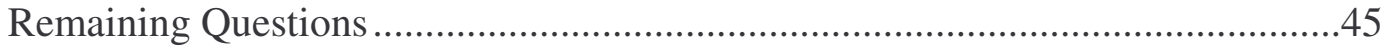

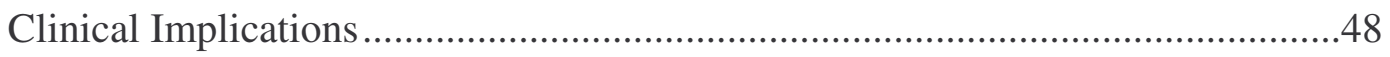

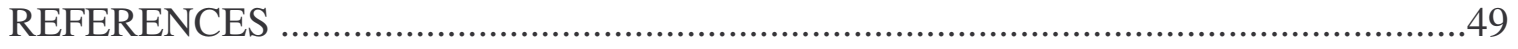

xii 
CHAPTER 3 ENVIRONMENTAL ENRICHMENT INCREASES PAIRED-PULSE

DEPRESSION IN RAT AUDITORY CORTEX .................................55

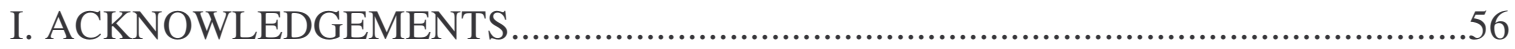

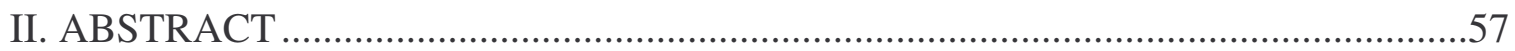

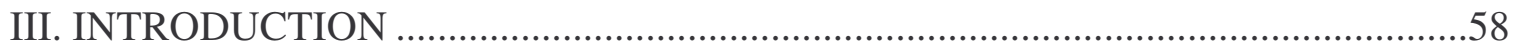

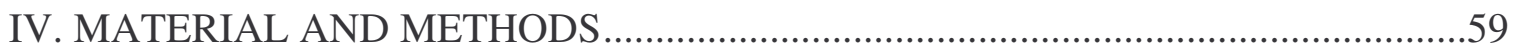

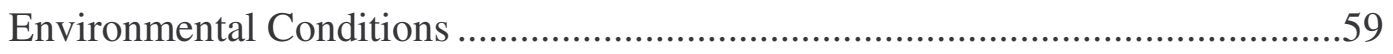

Experiment 1 - Extracellular Recordings ..................................................63

Acute Surgery ..............................................................................63

Stimulus Presentation and Data Analysis..........................................66

Experiment 2 - Evoked Potential Recordings ............................................67

Experimental Time-course ..........................................................67

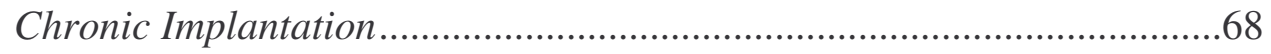

Stimulus Presentation and Data Analysis...........................................68

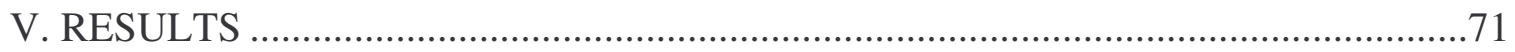

Experiment 1 - Extracellular Recordings .................................................. 71

Repetition Rate Transfer Functions ..................................................71 
Experiment 2 - Evoked Potential Recordings ..............................................76

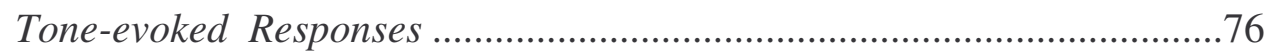

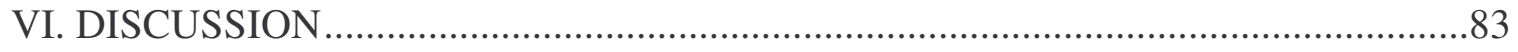

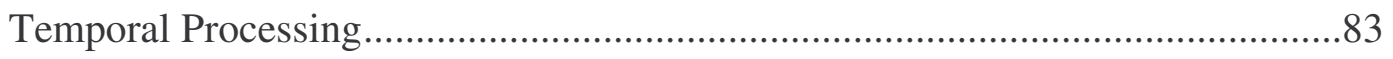

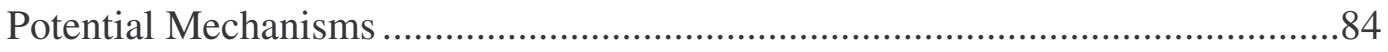

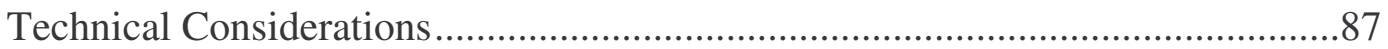

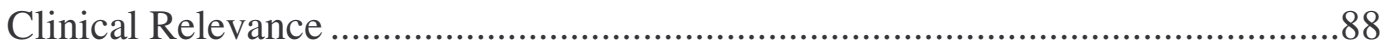

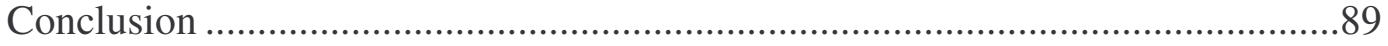

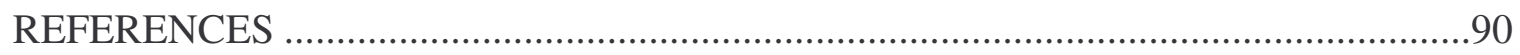

CHAPTER 4 A BRIEF REVIEW of 192 Ig-G SAPORIN..........................96

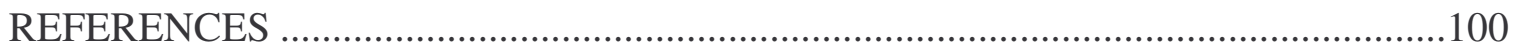

CHAPTER 5 SENSORY EXPERIENCE DETERMINES ENRICHMENT-INDUCED

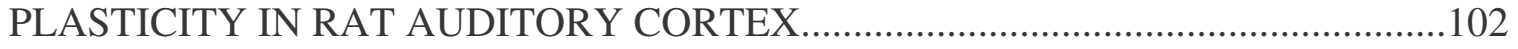

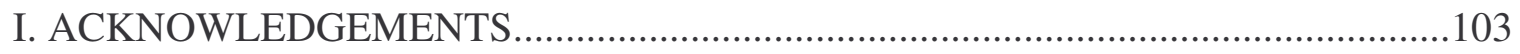

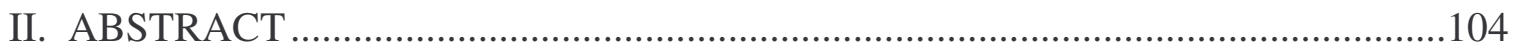

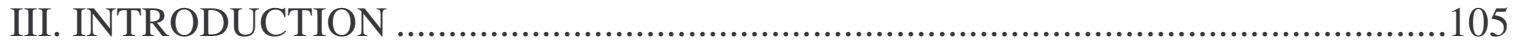

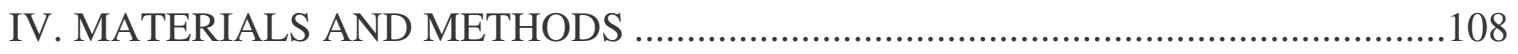




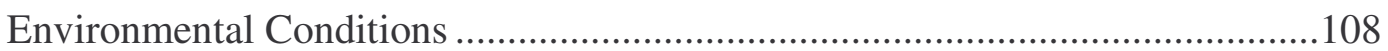

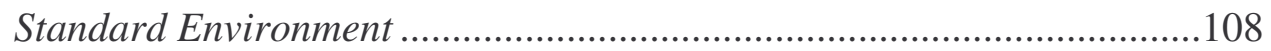

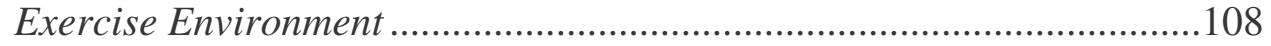

Social Environment ........................................................................108

Enriched Environment ...................................................................110

Auditory Exposure .............................................................................112

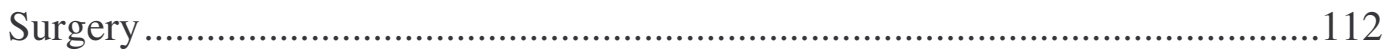

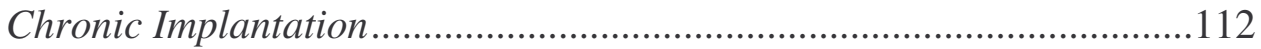

Intracerebroventricular Injection ..................................................113

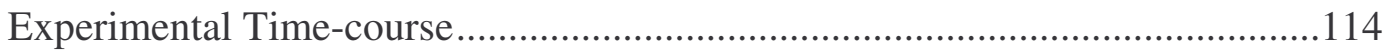

Environmental Factors Experiment ................................................114

Cholinergic Damage Experiment ...................................................114

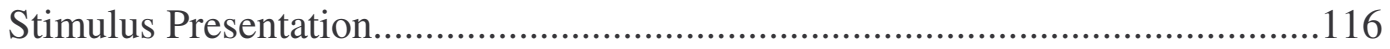

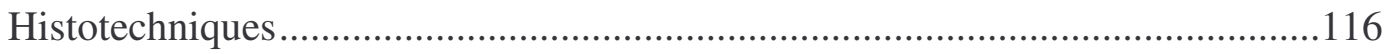

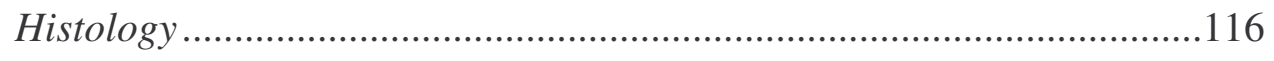

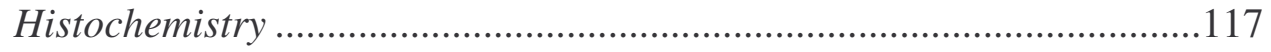


Analysis.

Response Strength

Paired-pulse Depression

Lesion Confirmation

V. RESULTS

Sham-Enriched

Cholinergic Damage

Environmental Factors

Tones vs. Noise Bursts.

VI. DISCUSSION

Technical Considerations

Clinical Implications .

Ceiling Effect

Conclusions

REFERENCES

CHAPTER 6 CONCLUSION.

REFERENCES

VITA

xvi 


\section{LIST OF FIGURES}

\section{CHAPTER 1}

1.1 Plasticity as a U-shaped function of environmental diversity ............... 7

\section{CHAPTER 2}

2.1 Schematic of standard and enriched housing conditions..................... 21

2.2 Experimental time-lines............................................. 22

2.3 Tone responses from an enriched rat.................................... 25

2.4 Mean noise burst post-stimulus time histograms for enriched and standard rats......................................................... 31

2.5 Grand mean average responses to noise for select weeks of the experiment .....36

2.6 Grand mean average responses to a $9-\mathrm{kHz}$ tone for select weeks of the experiment............................................................

2.7 Time-course of environmental plasticity................................ 39

2.8 Housing condition significantly affects strength of evoked potentials..........40

\section{CHAPTER 3}

3.1 Schematic of standard and enriched housing conditions.....................62

3.2 Experimental time-lines............................................. 65

3.3 Responses of a single site in an enriched rat to repeated tone and noise

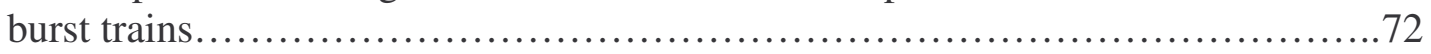

3.4 Repetition rate transfer function, vector strength, and Rayleigh statistic for tone trains ........................................................ 74

3.5 Repetition rate transfer function for noise bursts ......................... 75

3.6 Average tone-evoked response before, during, and after enrichment......... 77 
3.7 Average tone-evoked response for enriched compared to standard housed rats............................................................ 78

3.8 Overlays of the mean response evoked by each tone when presented in isolation or 500 to $50 \mathrm{~ms}$ after another tone.................................. 81

3.9 Average paired-pulse depression ratios for each group as a function of interstimulus interval.

\section{CHAPTER 4}

4.1 Nucleus basalis projections...........................................96

4.2 Intracerebroventricular injection...................................... 98

\section{CHAPTER 5}

5.1 Schematic of environmental housing conditions

5.2 Experimental time-lines

5.3 Mean auditory evoked potential, grand mean average, and plasticity index of sham-enriched group.

5.4 Environmental plasticity for sham-enriched, sham-standard, lesion-enriched, and lesion-standard groups.

5.5 Paired-pulse depression for sham-enriched, sham-standard, lesion-enriched, and lesion-standard groups.

5.6 Examples of AChE stained sections to verify cholinergic damage

5.7 Environmental plasticity index for exercise, social, and auditory exposure groups

5.8 Paired-pulse depression for exercise, social and auditory exposure groups.

5.9 Mean plasticity index of each group as a function of the mean pairedpulse ratio 
CHAPTER 6

6.1 Plasticity along a continuum of environmental interest and diversity........... 160 


\section{LIST OF TABLES}

\section{CHAPTER 2}

2.1 Response properties of AI neurons................................. 32

\section{CHAPTER 5}

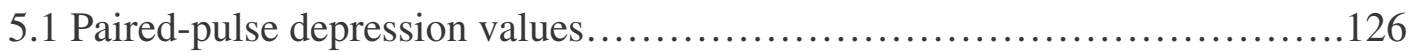

5.2 Plasticity index values for tones and noise bursts........................129 


\section{CHAPTER 1}

\section{INTRODUCTION}

Over the last 50 years, environmental enrichment has been shown to generate more than a dozen changes in brain anatomy and neurochemistry. For example, enrichment increases brain weight, cortical thickness, glial cell to neuron ratio, dendritic branching, number of synapses per neuron, and levels of neurotrophins compared to animals housed in standard laboratory conditions (Diamond et al., 1966, 1972; Bennett et al., 1964, 1969; Schapiro and Vukovich, 1970; Volkmar et al., 1972; Globus et al., 1973; Greenough et al., 1973; Por et al., 1982; Katz and Davies, 1984; Sirevaag et al., 1985; Turner and Greenough, 1985; Ickes et al., 2000; Naka et al., 2002). The anatomical and neurochemical consequences of enrichment on information processing have not been characterized, but may form the basis of physiological plasticity. Sensory enrichment, in the form of perceptual training, is an effective treatment for the temporal processing deficits associated with some central auditory processing disorders (Merzenich et al., 1996, 1999; Tallal et al., 1996; Hayes et al., 2003). These results suggest that focused and intensive enrichment may also improve the sensoryperceptual abnormalities associated with autism.

It is commonly believed that autism is a lifelong disability. Most treatments only modestly decrease autistic behaviors (Browning, 1971; Rutter et al., 1973; Lovaas et al., 1974, 1987; Mesibov et al., 1989; Venter et al., 1992; Gonzalez et al., 1993; McEachin et al., 1993; Shapiro et al., 1995; Coucouvanis, 1997; Smith et al., 1997; Rogers, 1998). During a period in which children typically acquire meaningful experiences, autistic 
symptoms may develop when a child is unable to understand the meaning of his experiences. Providing a rich and stimulating environment and facilitating the development of increasingly complex stages of play significantly advanced the treatment of autism (MacAlpine, 1998). Sequential play stages, beginning with sensory play and progressing through social and role-play, represent a child's ability to acquire meaning about the world. After several months of treatment, children typically advance to complex play stages, consistent with higher cognitive abilities, which coincide with a decrease in the severity of autistic symptoms. Moreover, these improvements are maintained long-term suggesting that the sensory neural circuits in the brain appropriately rewired. These results suggests that proper sensory development is necessary for higher cognitive processes, and that rich and stimulating environments facilitate recovery from neurological disability.

The experiments contained within this dissertation are based on a desire to bridge the gap between clinical and basic neuroscience that led to the development of a series of experiments using a simple and robust environmental enrichment paradigm. Auditory neuroscience was chosen as an area of interest to match themes initially brought to my attention during clinical training as a developmental therapist. Specifically, autism has been related to temporal lobe dysfunction and is associated with auditory sensory impairments, including hypo- and/or hyper- activity to sound (Koegel et al., 1976; Martineau et al., 1980; Wetherby et al., 1981; Courchesne et al., 1984; Konstantareas and Komatidis, 1987; Smith et al., 1988; Jure et al., 1991; George et al., 1992; Hoon et al., 1992; Klin, 1993; Tecchio et al., 2003; Gomes et al., 2004; Lepisto et al., 2005; Teder-Salejarvi et al., 2005). While 
there are several techniques available with which to study enrichment-induced plasticity, evoked potentials have the most clinical utility. In these experiments, I recorded evoked potentials to document plasticity resulting from a "spectrum" of environmental experiences. I aimed to document the physiological correlates of improved sensory processing and to investigate how neurons assign importance to sensory inputs in the environment-both of which may contribute to the decrease in clinical symptoms associated with play therapy.

Chapter 2 documents the consequences of enriched compared to standard housing on response strength of auditory cortex neurons and examines the time-course of these changes. In less than 2 weeks, evoked potentials of both young and adult rats increase during periods of housing in a stimulating environment and are degraded by exposure to a boring environment. Chapter 3 characterizes the effect of enrichment on temporal information processing in auditory cortex. Paired-pulse depression increases with days of environmental enrichment and is restored to control levels after return to standard housing conditions. Collectively, the results of chapters 2 and 3 suggest that the richness of the environment serves as a catalyst that increases neurophysiologic responses after being housed in the standard environment.

Since the enriched environment provides sensory stimulation and social interactions common in natural environments, the physiological consequences of enrichment may more accurately reflect normal cortical information processing. Conversely, rats in the standard environment exhibit reduced responses to sensory stimulation that fail to adapt at increased repetition rates, similar to individuals with autism (Buchwald et al., 1992). These differences may contribute to poor sensory processing, abnormal arousal levels, and inattentiveness 
characteristic of the autistic population. The observation that play-based therapy decreases autistic symptoms is consistent with the result that enrichment increases paired-pulse depression. If stimulation enhances cortical physiology in humans, these changes could provide the neurological foundation for therapy-based behavioral improvements. Future studies will be needed to determine if behavioral therapies for autism enhance cortical processing of sensory information.

Several disorders with sensory gating deficits also have cholinergic abnormalities, including individuals with autism (Buchwald et al., 1992; Perry et al., 2001). Since enrichment increases the levels of acetylcholine receptors, acetylcholinesterase, and choline acetyltransferase in multiple brain regions, in chapter 4 , we examined the effects of cholinergic modulation on enrichment-induced plasticity (Bennett et al., 1964; Por et al., 1982; Park et al., 1992). Cholinergic neurons of the nucleus basalis are activated as a function of the behavioral significance of stimuli, and since assigning meaning to sensory events in the environment is strongly related to clinical outcomes, we investigated cortical plasticity by selectively lesioning cholinergic projections from the nucleus basalis (Richardson and Delong, 1991; MacAlpine, 1998; Davis, 2000). Our results indicate that despite substantial cholinergic damage, enrichment increases response strength and pairedpulse depression in rat auditory cortex. Therefore, perceptual training may improve sensory gating abnormalities in autism and other clinical populations with cholinergic deficits.

Also in chapter 4, we examined the influence of physical activity, social stimulation, and auditory exposure on enrichment-induced plasticity. Since exercise and social stimulation can independently generate many of the benefits that accompany general 
enrichment, we investigated whether these manipulations experienced in isolation are sufficient to increase response strength and paired-pulse depression in auditory cortex (Rosenzweig et al., 1978; Einon et al., 1981; Turner and Greenough, 1985; Black et al., 1990; Geyer et al., 1993; Stummer et al., 1994; Neeper et al., 1996; van Praag et al., 1999; Anderson et al., 2002; Risedal et al., 2002; Gordon et al., 2003; Swain et al., 2003; Farmer et al., 2004; Preece et al., 2004). Whereas rats that could hear the enriched environment exhibited enhanced cortical responses and increased paired-pulse depression similar to rats housed in the enrichment cage, social housing and physical activity had no effect on cortical physiology compared to enrichment. If cortical physiology leads to changes in behavior, these results would suggest that neither the motor activities nor social interactions associated with play therapy are critical for behavioral improvements. However, rats housed in a social environment exhibited more paired-pulse depression than rats housed with a running wheel, and were not different than rats that observed the enriched environment. These results demonstrate that sensory experience facilitates physiological enrichment-induced plasticity, but that temporal plasticity may exist along a continuum of experiences.

In summary, cortical plasticity may exist as a function of environmental diversity (Figure 1.1). On one end of the continuum, are environments that are predictable, boring, and do not command attention, and as a consequence, yield no plasticity. At the other end, are environments with sensory stimuli that are random, meaningless, and disconnected from attention. The optimal amount of plasticity (increased response strength and paired-pulse depression) would result in an environment that contained rich and diverse sensory stimuli to which behavioral consequences were attached and attention was regulated. If this prediction 
were true, it might mean that children with autism experience the world as a series of random, meaningless, unconnected sensory events. Since enrichment may more closely approximate the level of sensory stimulation and social interactions common in everyday life, enriching sensory-based therapies may decrease autistic symptoms by increasing response strength and paired-pulse depression. Future studies are needed to determine the perceptual consequences of increased response strength and paired-pulse depression in children with autism.

In conclusion, the collective dissertation effectively demonstrates that physiological cortical plasticity is determined by the current housing condition. Understanding the rules that govern how sensory experience guides neural plasticity will continue to advance the development of effective neurorehabilitation strategies. 


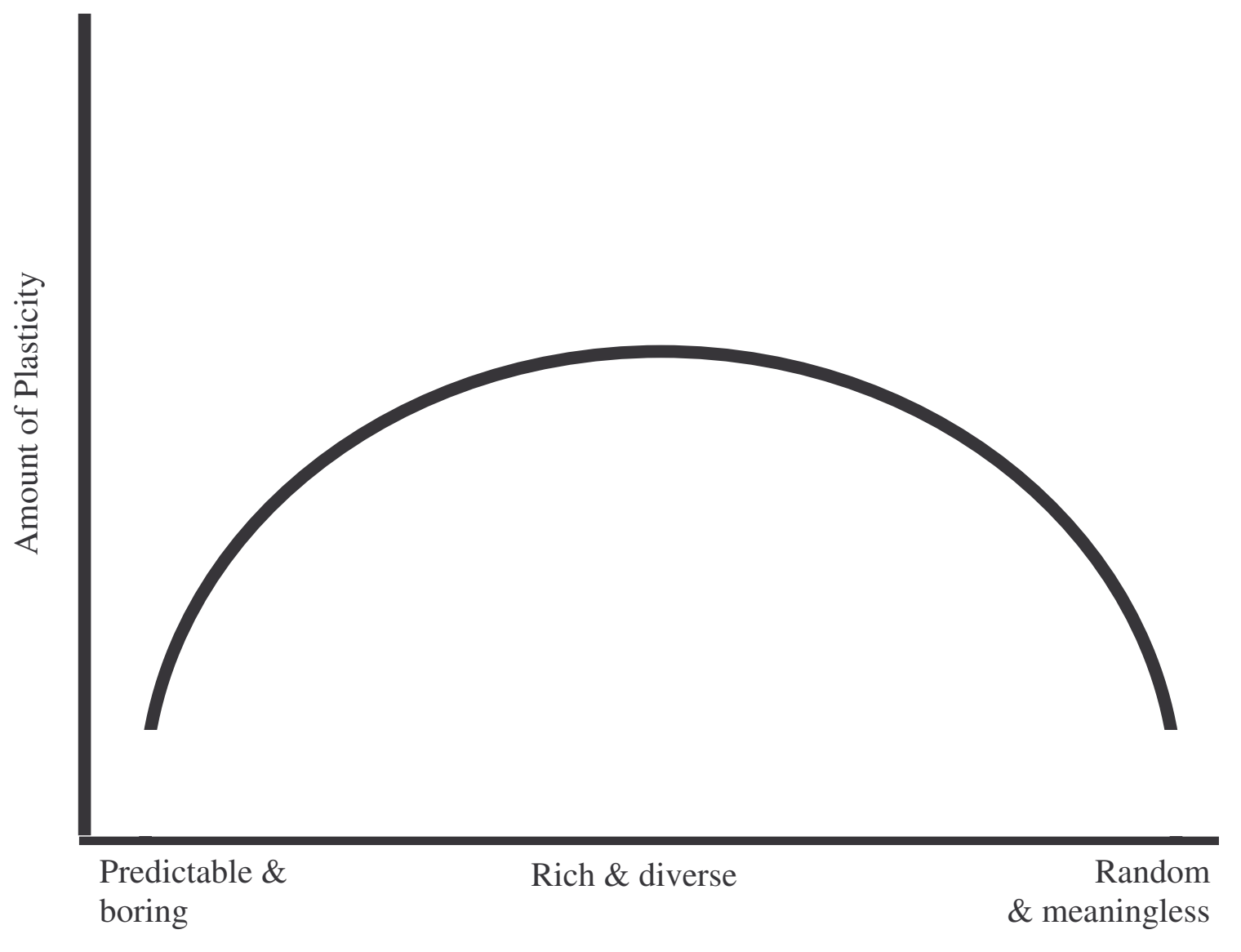

Environment

Figure 1.1. Plasticity as a U-shaped function of environmental diversity. Environments exist along a continuum of meaningful and interesting sensory interactions. Rich, stimulating environments increase response strength and paired-pulse depression. 


\section{REFERENCES}

Anderson, M.J. and Micheli-Tzanakou, E. (2002). Auditory stimulus optimization with feedback from fuzzy clustering of neuronal responses. IEEE Trans Inf Technol Biomed 6(2): 159-70.

Bear, M.F. and Singer, W. (1986). Modulation of visual cortical plasticity by acetylcholine and noradrenaline. Nature 320(6058): 172-6.

Bennett, E.L., Krech, D. and Rosenzweig, M.R. (1964). Reliability and regional specificity of cerebral effects of environmental complexity and training. J Comp Physiol Psyc 57: 440-441.

Bennett, E.L., Rosenzweig, M.R. and Diamond M.C. (1969). Rat brain: effects of environmental enrichment on wet and dry weights. Science 163: 825-826.

Black, J.E., Isaacs, K. R., Anderson, B.J., Alcantara, A.A. and Greenough W.T. (1990). Learning causes synaptogenesis, whereas motor activity causes angiogenesis, in cerebellar cortex of adult rats. PNAS 87(14): 5568-72.

Browning, R.M. (1971). Treatment effects of a total behavior modification program with five autistic children. Behav Res Ther 9(4): 319-27.

Buchwald, J.S., Erwin, R, Van Lancker, D, Guthrie, D, Schwafel, J. and Tanguay, P. (1992). Midlatency auditory evoked responses: P1 abnormalities in adult autistic subjects. Electroencephalogr Clin Neurophysiol 84: 164-171.

Coucouvanis, J. (1997). Behavioral intervention for children with autism. J Child Adolesc Psychiatr Nurs 10(1): 37-44.

Courchesne, E., Kilman, B.A., Galambos, R. and Lincoln, A.J. (1984). Autism: processing of novel auditory information assessed by event-related brain potentials. Electroencephalogr Clin Neurophysiol 59(3): 238-48.

Davis, A.E. (2000). Cognitive impairments following traumatic brain injury. Etiologies and interventions. Crit Care Nurs Clin North Am 12(4): 447-56.

Diamond, M.C., Law, F., Rhodes, H., Linder, B., Rosenzweig, M.R., Krech, D. and Bennett, E.L. (1966). Increases in cortical depth and glia numbers in rats subjected to enriched environment. J Comp Neurol 128(1): 117-26. 
Diamond, M.C., Rosenzweig, M.R., Bennett, E.L., Linder, B. and Lyon L. (1972). Effects of environmental enrichment and impoverishment on rat cerebral cortex. J Neurobiol 3(1): 47-64.

Einon, D.F., Humphreys, A.P., Chivers S.M., Field, S. and Naylor, V. (1981). Isolation has permanent effects upon the behavior of the rat, but not the mouse, gerbil, or guinea pig. Dev Psychobiol 14(4): 343-55.

Farmer, J., Zhao, X., van Praag, H. Wodtke, R., Gage F.H. and Christie B.R. (2004). Effects of voluntary exercise on synaptic plasticity and gene expression in the dentate gyrus of adult male Sprague-Dawley rats in vivo. Neurosci 124(1): 71-9.

George, M.S., Costa, D.C., Kouris K., Ring, H.A. and Ell, P.J. (1992). Cerebral blood flow abnormalities in adults with infantile autism. J Nerv Ment Dis 180(7): 413-7.

Geyer, M.A., Wilkinson, L.S., Humby, T. Robbins, T.W. (1993). Isolation rearing of rats produces a deficit in prepulse inhibition of acoustic startle similar to that in schizophrenia. Biol Psych 34(6): 361-72.

Globus, A., Rosenzweig, M.R., Bennett, E.L. and Diamond, M.C. (1973). Effects of differential experience on dendritic spine counts in rat cerebral cortex. J Comp Physiol Psyc 82(2): 175-81.

Gomes, E., Rotta, N.T., Pedrosa, F.S., Sleifer, P. and Danesi, M.C. (2004). Auditory hypersensitivity in children and teenagers with autistic spectrum disorder. Arq Neuropsiquiatr 62(3B): 797-801.

Gonzalez, N.M., Alpert, M., Shay, J., Campbell, M. and Small, A.M. (1993). Autistic children on follow-up: change of diagnosis. Psychopharmacol Bull 29(3): 353-8.

Gordon, N.S., Burke, S., Akil, H., Watson, S.J. and Panksepp, J. (2003). Socially-induced brain 'fertilization': play promotes brain derived neurotrophic factor transcription in the amygdala and dorsolateral frontal cortex in juvenile rats. Neurosci Lett 341(1): 17-20.

Greenough, W.T. and Volkmar, F.R. (1973). Pattern of dendritic branching in occipital cortex of rats reared in complex environments. Exp Neurol 40(2): 491-504.

Hayes, E.A., Warrier C.M., Nicol, T.G., Zecker, S.G. and Kraus, N. (2003). Neural plasticity following auditory training in children with learning problems. Clin Neurophysiol. 114(4): 673-684. 
Hoon, A.H., Jr. and Reiss, A.L. (1992). The mesial-temporal lobe and autism: case report and review. Dev Med Child Neurol 34(3): 252-9.

Ickes, B.R., Pham, T.M., Sanders, L.A., Albeck, D.S., Mohammed, A.H. and Granholm, A.C. (2000). Long-term environmental enrichment leads to regional increases in neurotrophin levels in rat brain. Exp Neurol 164: 45-52.

Jure, R., Rapin, I. and Tuchman, R.F. (1991). Hearing-impaired autistic children. Dev Med Child Neurol 33(12): 1062-72.

Katz, H.B. and Davies, C.A. (1984). Effects of differential environments on the cerebral anatomy of rats as a function of previous and subsequent housing conditions. Exp Neurol 83: 274-287.

Klin, A. (1993). Auditory brainstem responses in autism: brainstem dysfunction or peripheral hearing loss? J Autism Dev Disord 23(1): 15-35.

Koegel, R.L. and Schreibman, L. (1976). Identification of consistent responding to auditory stimuli by a functionally "deaf" autistic child. J Autism Child Schizophr 6(2): 147-56.

Konstantareas, M.M. and Komatidis, S. (1987). Brief Report: Ear Infections in Autistic and Normal Children. J of Autism and Dev Dis 17(4): 585-594.

Lepisto, T., Kujala, T., Vanhala, R., Alku, P., Huotilainen, M. and Naatanen, R. (2005). The discrimination of and orienting to speech and non-speech sounds in children with autism. Brain Res 1066(1-2): 147-57.

Lovaas, O.I. (1987). Behavioral treatment and normal educational and intellectual functioning in young autistic children. J Consult Clin Psychol 55(1): 3-9.

Lovaas, O.I., Schreibman, L. and Koegel, R.L. (1974). A behavior modification approach to the treatment of autistic children. J Autism Child Schizophr 4(2): 111-29.

MacAlpine, M.L. (1998). Microdevelopment in autism: The power of play. Ph.D. diss., University of Texas at Dallas.

Martineau, J.F., Laffont, F., Bruneau, N., Roux, S and Lelord, G. (1980). Event-related potentials evoked by sensory stimulation in normal mentally retarded and autistic children. Electroencephalogr Clin Neurophysiol 48(2): 140-53. 
McEachin, J.J., Smith, T. and Lovaas, O.I. (1993). Long-term outcome for children with autism who received early intensive behavioral treatment. Am J Ment Retard 97(4): 359-91.

Merzenich, M.M., Jenkins, W.M., Johnston, P., Schreiner, C., Miller, S.L. and Tallal, P. (1996). Temporal processing deficits of language-learning impaired children ameliorated by training. Science 271(5245): 77-81.

Merzenich, N.N., Sauders, G., Jenkins, W.M., Miller, S., Peterson, B. and Tallal, P. (1999). Pervasive developmental disorders: Listening training and language abilities. In The changing nervous system: Neurobehavioral consequences of early brain disorders, ed. SH Broman and JM Fletcher, 365-88. New York: Oxford University Press.

Mesibov, G.B., Schopler, E., Schaffer, B. and Michal, N. (1989). Use of the childhood autism rating scale with autistic adolescents and adults. J Am Acad Child Adolesc Psychiatry 28(4): 538-41.

Naka, F., Shiga, T., Yaguchi, M. and Okado, N. (2002). An enriched environment increases noradrenaline concentration in the mouse brain. Brain Res 924(1): 124-6.

Neeper, S.A., Gomez-Pinilla, F., Choi, J. and Cotman C.W. (1996). Physical activity increases mRNA for brain-derived neurotrophic factor and nerve growth factor in rat brain. Brain Res 726(1-2): 49-56.

Park, G.A., Pappas, B.A., Murtha, S.M. and Ally A. (1992). Enriched environment primes forebrain choline acetyltransferase activity to respond to learning experience. Neurosci Lett 143(1-2): 259-62.

Por, S.B., Bennett, E.L. and Bondy, S.C. (1982). Environmental enrichment and neurotransmitter receptors. Behav Neural Biol 34: 132-140.

Perry, E.K., Lee, M.L., Martin-Ruiz, C.M., Court, J.A., Volsen, S.G., Merrit, J., Folly, E., Iversen, P.E., Bauman, M.L., Perry, R.H. and Wenk, G.L. (2001). Cholinergic activity in autism: abnormalities in the cerebral cortex and basal forebrain. Am J Psychiatry 158: 1058-1066.

Preece, M.A., Dalley, J.W., Theobald, D.E., Robbins, T.W. and Reynolds, G.P. (2004). Region specific changes in forebrain 5-hydrox ytryptamine1A and 5hydroxytryptamine $2 \mathrm{~A}$ receptors in isolation-reared rats: an in vitro autoradiography study. Neurosci 123(3): 725-32. 
Richardson, R.T. and DeLong, M.R. (1991). Electrophysiological studies of the functions of the nucleus basalis in primates. Adv Exp Med Biol 295: 233-252.

Risedal, A., Mattsson, B., Dahlqvist, P., Nordborg, C., Olsson, T. and Johansson, B.B. (2002). Environmental influences on functional outcome after a cortical infarct in the rat. Brain Res Bull 58(3): 315-21.

Rogers, S.J. (1998.) Empirically supported comprehensive treatments for young children with autism. J Clin Child Psychol 27(2): 168-79.

Rosenzweig, M.R., Bennett, E.L., Herbert, M. and Morimoto, H. (1978). Social grouping cannot account for cerebral effects of enriched environments. Brain Res 153(3): 56376.

Rutter, M. and Bartak, L. (1973). Special educational treatment of autistic children: a comparative study. II. Follow-up findings and implications for services. J Child Psychol Psychiatry 14(4): 241-70.

Schapiro, S. and Vukovich, K.R. (1970). Early experience effects upon cortical dendrites: a proposed model for development. Science 167(916): 292-4.

Shapiro, T. and Hertzig, M. (1995). Applied behavioral analysis: astonishing results? J Am Acad Child Adolesc Psychiatry 34(10): 1255-6.

Sirevaag, A.M. and Greenough, W.T. (1985). Differential rearing effects on rat visual cortex synapses. II. Synaptic morphometry. Brain Res 351(2): 215-26.

Smith, D.E., Miller, S.D., Stewart, M., Walter, T.L. and McConnell, J.V. (1988). Conductive hearing loss in autistic, learning-disabled, and normal children. J Autism Dev Disord 18(1): 53-65.

Smith, T., Eikeseth, S., Klevstrand, M. and Lovaas O.I. (1997). Intensive behavioral treatment for preschoolers with severe mental retardation and pervasive developmental disorder. Am J Ment Retard 102(3): 238-49.

Stummer, W., Weber, K., Tranmer, B., Baethmann, A., Kempski, O. (1994). Reduced mortality and brain damage after locomotor activity in gerbil forebrain ischemia. Stroke 25(9): 1862-9.

Swain, R.A., Harris, A.B., Wiener E.C., Dutka, M.V., Morris, H.D., Theien, B.E., Konda, S., Engberg, K., Lauterbur, P.C. and Greenough W.T. (2003). Prolonged exercise induces angiogenesis and increases cerebral blood volume in primary motor cortex of the rat. Neurosci117(4): 1037-46. 
Tallal, P., Miller, S.L., Bedi, G., Byma, G., Wang, X., Nagarajan, S.S., Schreiner, C., Jenkins, W.M. and Merzenich M.M. (1996). Language comprehension in languagelearning impaired children improved with acoustically modified speech. Science 271(5245): 81-4.

Tecchio, F., Benassi, F., Zappasodi, F., Gialloreti, L.F., Palermo, M., Seri, S. and Rossini, P.M. (2003). Auditory sensory processing in autism: a magnetoencephalographic study. Biol Psychiatry 54(6): 647-54.

Teder-Salejarvi, W.A., Pierce, K.L., Courchesne, E. and Hillyard, S.A. (2005). Auditory spatial localization and attention deficits in autistic adults. Brain Res Cogn Brain Res 23(2-3): 221-34.

Turner, A.M. and Greenough, W.T. (1985). Differential rearing effects on rat visual cortex synapses. I. Synaptic and neuronal density and synapses per neuron. Brain Res 329(12): 195-203.

van Praag, H., Kempermann G. and Gage F.H. (1999). Running increases cell proliferation and neurogenesis in the adult mouse dentate gyrus. Nat Neurosci. 2(3): 266-70.

Venter, A., Lord, C. and Schopler, E. (1992). A follow-up study of high-functioning autistic children. J Child Psychol Psychiatry 33(3): 489-507.

Volkmar, F.R. and Greenough, W.T. (1972). Rearing complexity affects branching of dendrites in the visual cortex of the rat. Science 176(42): 1145-7.

Wetherby, A.M., Koegel, R.L., Mendel, M. (1981). Central auditory nervous system dysfunction in echolalic autistic individuals. J Speech Hear Res 24(3): 420-9. 


\title{
CHAPTER 2
}

\section{ENVIRONMENTAL ENRICHMENT IMPROVES RESPONSE STRENGTH, THRESHOLD, SELECTIVITY, AND LATENCY OF AUDITORY CORTEX NEURONS}

\author{
Navzer D. Engineer*, Cherie R. Percaccio *, Pritesh K. Pandya, Raluca Moucha, Daniel L. \\ Rathbun, Michael P. Kilgard \\ * These authors contributed equally to this work \\ School of Behavioral and Brain Sciences, GR41
}

The University of Texas at Dallas

P.O. Box 830688

Richardson, Texas 75083

Keywords: critical period, developmental plasticity, neurophysiology, receptive fields, sensory processing, social isolation 


\section{ACKNOWLEDGEMENTS}

Navzer D. Engineer is the primary author of Experiment 1. Pritesh K. Pandya, Raluca Moucha, Daniel L. Rathbun, and Michael P. Kilgard assisted Navzer with surgeries, data collection, and analysis. Cherie R. Percaccio is the primary author of Experiment 2. Michael P. Kilgard assisted Cherie with analysis. The authors thank Wei Wei Dai for assistance with recording sessions and animal care colony management. This work was supported by the Cure Autism Now Foundation and the Callier Excellence in Education Fund. 


\section{ABSTRACT}

Over the last 50 years, environmental enrichment has been shown to generate more than a dozen changes in brain anatomy. The consequences of these physical changes on information processing have not been well studied. This report details seven significant, new findings about the influence of housing conditions on the responses of rat auditory cortex neurons. First, enrichment dramatically increases the strength of cortical responses. Tone-evoked potentials of enriched rats, for example, were more than twice the amplitude of rats raised in standard laboratory conditions. Second, cortical responses of both young and adult animals benefit from exposure to an enriched environment and are degraded by exposure to an impoverished environment. Third, housing condition significantly affected cortical responses in less than 2 weeks. Fourth, recordings made under anesthesia indicate that enrichment increases the number of neurons activated by any sound. This finding demonstrates that the differences in evoked potentials recorded from awake rats were not due to differences in behavioral state. Finally, enrichment made A1 neurons more sensitive to quiet sounds, and more selective for tone frequency. These experiments provide the first evidence of physiologic changes in auditory cortex processing resulting from generalized environmental enrichment. 


\section{INTRODUCTION}

Environmental enrichment results in morphologic, molecular and physiologic changes in sensory and motor cortices of young and adult animals (van Praag et al., 2000; Diamond, 2001). Rats raised in enriched conditions exhibit increases in cortical thickness, gene expression, acetylcholinesterase levels, oligodendrocyte to neuron ratio, dendritic branching, and number of synapses per neuron compared to animals raised in standard conditions (Bennett et al., 1966; Diamond et al., 1966; Schapiro and Vukovich, 1970; Diamond et al., 1972; Volkmar and Greenough, 1972; Globus et al., 1973; Greenough et al., 1973; Globus et al., 1973; Sirevaag and Greenough, 1987; Rampon et al., 2000; Hilbig et al., 2002; Staiger et al., 2002).

Neurophysiologic responses can also be altered by experience. Cats raised in enriched conditions have sharper orientation tuning in primary visual cortex and are able to resolve higher spatial frequencies compared to cats raised in standard conditions (Beaulieu and Cynader, 1990). Environmental enrichment narrows receptive fields and sharpens the topographic organization of adult rat primary somatosensory cortex (Coq and Xerri, 1998). Experience-dependent remodeling of receptive fields and topographic organization of auditory cortex has also been observed in behaviorally trained animals (Ahissar and Ahissar, 1994; Weinberger and Bakin, 1998; Edeline, 1999). Practice on frequency discrimination improves behavioral performance, narrows receptive fields, and expands the region of A1 responding to the trained frequency (Recanzone et al., 1993). The effects of more generalized enrichment have not been reported in auditory cortex. 
In the present study, our aims were to document the consequences of environmental enrichment on response properties of auditory cortex neurons and examine the time-course of these changes. Evoked potentials from awake rats and extracellular recordings from anesthetized rats show significant changes in response strength, receptive field characteristics and temporal response properties. 


\section{MATERIALS AND METHODS Environmental Conditions}

Thirty-seven female Sprague-Dawley rats were used in this study. The standard housing condition consisted of 1-2 rats per cage (Figure 2.1 A). In the enriched environment, 4-8 rats were housed together in a single large cage in a separate room from the main rat colony at the University of Texas at Dallas (Figure 2.1 B).

The enriched environment consisted of a large cage $(45 \mathrm{~L} \mathrm{x} 76 \mathrm{~W}$ x $90 \mathrm{H} \mathrm{cm})$ of four levels connected by ramps. Touch plates at the bottom of two ramps triggered different tones $(2100$ or $4000 \mathrm{~Hz}$ ) when the rat stepped on the plates. In addition, chains, wind chimes, or bells were hung across the entrance of each ramp so that a unique sound was elicited when rats passed from one level to the next. A motion detector emitted an electronic chime each time a rat crossed the infrared beam in front of the water source. An exercise wheel emitted a tone (3000 Hz Piezo Speaker) and activated a small green light emitting diode with each rotation. Each movement-triggered sound had unique spectral and temporal characteristics that provided behaviorally meaningful information about the location and activity of other rats in the cage. The power spectrum of these sounds spanned the entire hearing range of the rat (1-45 kHz). All sound intensities were less than $75 \mathrm{~dB}$ SPL.

A CD player presented randomly selected sounds every 2 to 60 seconds. These sounds included simple tones, amplitude modulated and frequency modulated tones, noise bursts and other complex sounds (rat vocalizations, classical music, rustling leaves, etc.). Seven of the seventy-four sounds activated a pellet dispenser (Med Associates) that delivered 
a sugar pellet to encourage attention to the sounds. The rewarded tracks included modulated tones with different carrier frequencies and frequency modulated sweeps. The sound sources added to the enriched environment were provided 24 hours per day. After one month, a vasectomized male rat was introduced in the enriched cage to encourage more natural social interactions appropriate for these ages since it is known that rats reach sexual maturation by 8-12 weeks of age.

The acoustic environment of the standard condition consisted of vocalizations from 20-30 other rats housed in the same room, and sounds resulting from daily room traffic, feeding and cleaning. The enriched group also heard sounds caused by room traffic, feeding, cleaning and animal vocalization; however, sounds in the enriched condition were more diverse and provided more behaviorally relevant information than the sounds in the standard condition. Rats in both conditions were on a reverse 12-hour light/dark cycle. As a result, both groups heard the sounds of room traffic while they were most active.

For both housing conditions, constant temperature and humidity were maintained. Food and water were provided ad libium for all rats. All the animals used in this study were housed with their mothers and littermates until weaning at 4 weeks of age. 


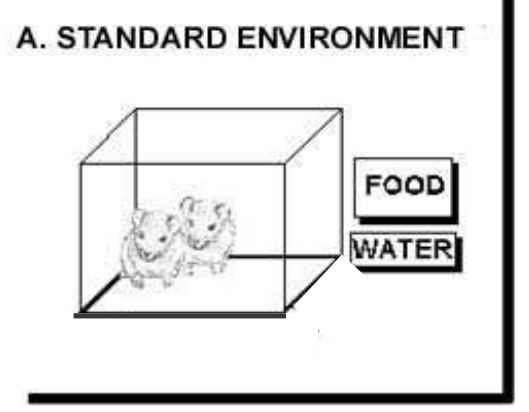

B. ENRICHED ENVIRONMENT
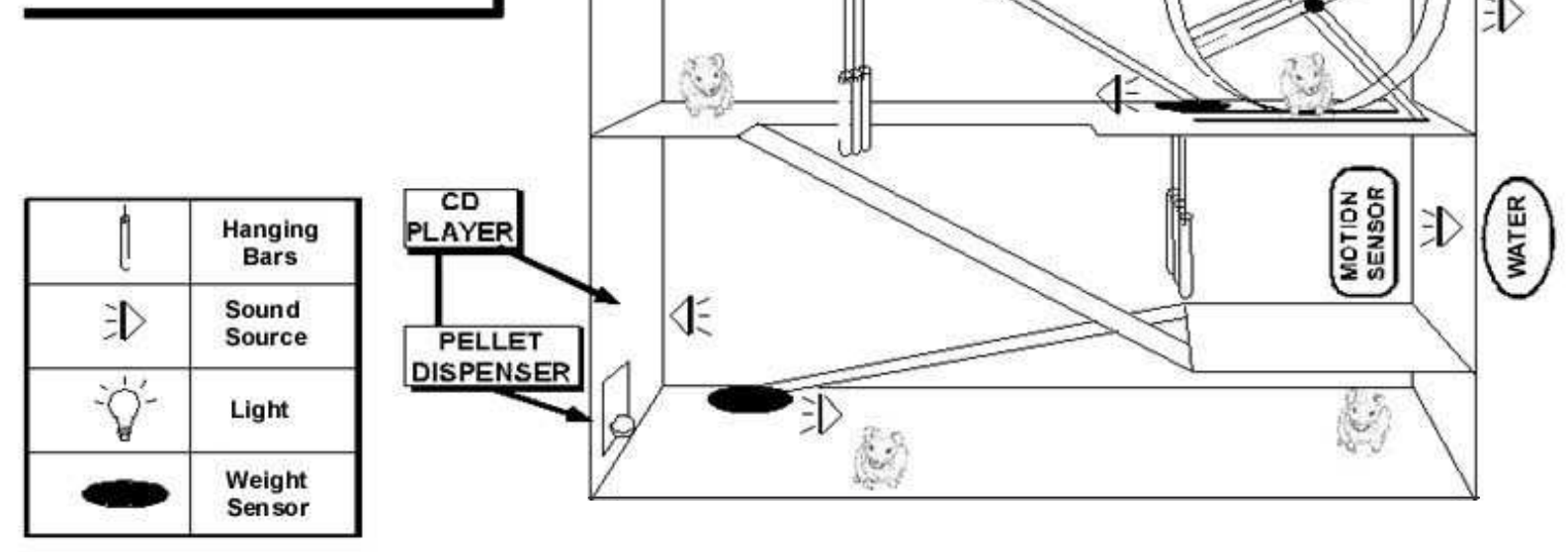

Figure 2.1. Schematic of standard and enriched housing conditions. A) The standard condition consisted of 1 or 2 rats housed in hanging cages within an animal colony room. B) The enriched condition consisted of 4-8 rats housed in a rich environment with devices that generated different sounds when rats crossed a motion detector path, stepped on weight sensors, or passed through hanging bars. In addition, each rotation of the running wheel triggered a brief tone and light flash, and a CD player played 74 sounds, including tones, noise bursts, musical sequences and other complex sounds, in random order. Some of these sounds were associated with delivery of a sugar reward. While the sounds in the enriched environment were more diverse and behaviorally relevant, rats in both conditions heard approximately the same number of sounds each day. 


\section{Experiment 1 - Extracellular Recordings}

Thirty-day-old female Sprague-Dawley rats were randomly assigned to either the enriched environment $(n=8)$ or the standard condition $(n=8)$. The enriched rats were raised 4 per cage (in two sessions), while rats in the standard condition were raised 2 per cage. Acute microelectrode mapping was performed after 8 weeks in each environment (Figure 2.2 A, B).

\section{Age (weeks)}

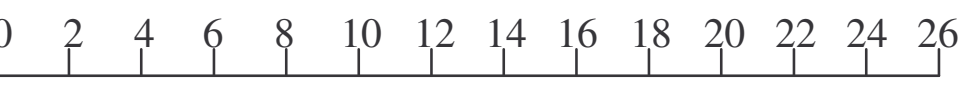
A) Experiment 1a
B) Experiment $1 b$
C) Experiment 2a
D) Experiment $2 b$
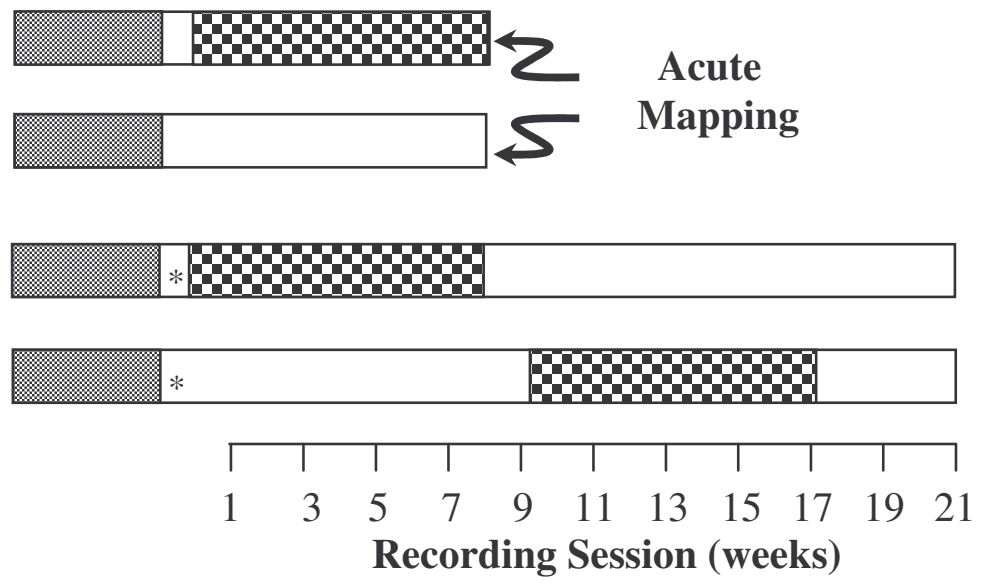

Housed w/
mother and
littermates $\quad \begin{aligned} & \text { Standard } \\ & \text { Environment }\end{aligned}$

Figure 2.2. Experimental time-lines. A\&B) Experiment 1: Extracellular recordings were compared from rats housed in the enriched environment or the standard condition for 8 weeks. C\&D) Experiment 2: Evoked potentials from auditory cortex were recorded weekly for five months. Asterisks indicate chronic electrode implantation. In Experiment 2a (earlyenriched), rats were housed in the enriched condition for 8 weeks (from 5-13 weeks of age), and then switched to the standard condition and housed singly until 26 weeks of age. In Experiment $2 b$ (late-enriched), rats were housed in the standard condition for 9 weeks, then switched to the enriched condition for 8 weeks (from 14-22 weeks of age), and finally, back to the standard condition until 26 weeks of age. 
Acute Surgery

Surgical anesthesia was induced with sodium pentobarbital (i.p., $50 \mathrm{mg} / \mathrm{kg}$ ). A state of areflexia was maintained throughout the surgery and recording phases with supplemental doses of dilute pentobarbital ( $8 \mathrm{mg} / \mathrm{ml}$; 0.2-0. $5 \mathrm{ml}$ i.p). The interval between supplements varied depending on the anesthetic state of the animal but was typically every 1-1.5 hr. Anesthesia depth was evaluated by heart rate, breathing rate, toe-pinch responses and corneal reflexes. These indicators were indistinguishable between the two groups. Circulatory function was monitored with EKG and pulse oximetry. Fluid balance was maintained with a 1:1 mixture of $5 \%$ Dextrose and Ringer's Lactate $(\sim 0.5 \mathrm{ml} / \mathrm{hour})$. Body temperature was maintained at $37^{\circ} \mathrm{C}$. The trachea was cannulated to minimize breathing sounds and ensure adequate ventilation. Humidified air was delivered to the open end of the cannula. After the cisterna magnum was drained to minimize cerebral edema, the right auditory cortex was exposed and the dura resected. The cortex was maintained under a layer of viscous silicon oil to prevent desiccation during the 24-30 hr experiment and a detailed map of auditory cortex was generated from 50-100 microelectrode penetrations. Parylene coated tungsten microelectrodes (FHC) were lowered $550 \mu \mathrm{m}$ below the pial surface (layer $4 / 5$ ) of the right auditory cortex. Spikes from a small cluster of neurons were collected at each penetration site. Penetration locations were referenced using cortical vasculature as landmarks. 
Stimulus Presentation and Data Analysis

Auditory stimuli were delivered from the left side of the rat via a calibrated speaker in a shielded, double-walled sound attenuating chamber and were generated using Brainware (Tucker-Davis Technologies). Auditory frequency tuning curves were determined at each site by presenting 81 logarithmically spaced frequencies from 1 to $32 \mathrm{kHz}$ at 16 intensities from 0 to $75 \mathrm{~dB}$ (1296 total stimuli). The tones were randomly interleaved and separated by $500 \mathrm{~ms}$. In addition, responses to $70 \mathrm{~dB}$ noise burst trains (4 rates $\mathrm{X} 12$ repetitions) were also recorded at each penetration site. A post-stimulus time histogram (PSTH) was created by summing spikes resulting from the repeated presentation of a single noise burst at each site. All stimuli were $25 \mathrm{~ms}$ long with $3 \mathrm{~ms}$ rise and fall time. Action potentials were recorded simultaneously from two Parylene coated tungsten microelectrodes (2 M $\Omega$ ). The neural signals were filtered $(0.3-15 \mathrm{kHz})$ and amplified $(10,000 \mathrm{X})$. Action potential waveforms were recorded whenever a set threshold was exceeded.

Tuning curve parameters were defined by an experienced blind observer using custom software that randomized the order of data from each recording site across both groups. Best frequency (BF), bandwidth measures (BW), response threshold, spontaneous rate, and latency measurements for each penetration were recorded (Figure 2.3). The BF is the frequency that evokes a reliable response at the lowest intensity (response threshold). Frequency bandwidth is the range of frequencies that each site responds to at 10, 20, 30 and $40 \mathrm{~dB}$ above threshold. First spike latency is the time from stimulus onset to the earliest reliable neural response. The end of response was defined as the time after tone onset when 
the PSTH created by summing the responses to all of the tones within each site's tuning curve returned to baseline. The borders of A1 were defined based on continuous topography of CF and short response latency. Sites with high thresholds, long latencies, broad tuning and discontinuities in CF topography were considered non-A1 (Kilgard, et al., 2001).
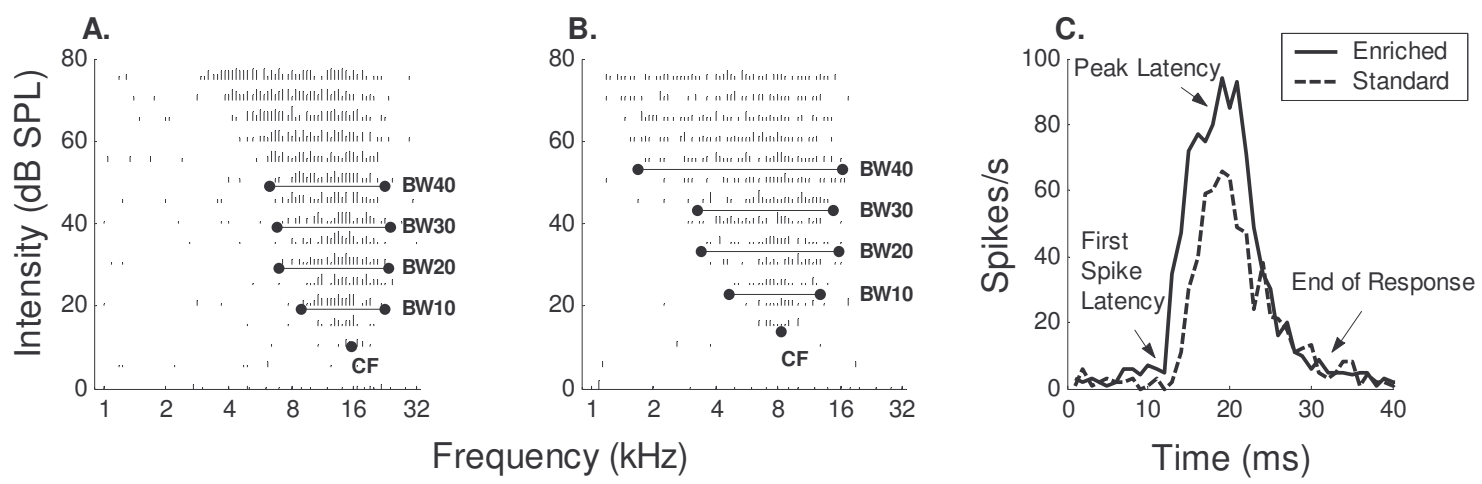

Figure 2.3. Tone responses from an enriched rat (A) show more spikes per tone, narrower tuning and lower thresholds compared to tone responses from a standard rat (B). C) PSTH's for these two sites illustrate the greater response strength after enrichment, later peak latency, and end of response latency.

\section{Experiment 2 - Auditory Evoked Potential}

\section{Experimental Time-course}

Evoked potentials were recorded each week from an electrode implanted over left auditory cortex to determine the time-course of environmental plasticity. Twenty-one female Sprague-Dawley rats were randomly assigned to one of two groups. Rats in Experiment 2a $(n=12)$ were housed in the enriched environment for 8 weeks (from 5-13 weeks of age), and then moved to the standard condition and housed singly until 26 weeks of age (Figure 2.2 C). Rats in Experiment 2b (n=9) were housed singly in the standard condition for 9 weeks, then 
moved to the enriched environment for 8 weeks (14-22 weeks of age), and finally, back to the standard condition until 26 weeks of age (Figure 2.2 D).

\section{Chronic Implantation}

Rats were chronically implanted at 28 days of age with a ball electrode over AI and a ground screw over the cerebellum. Surgical anesthesia was induced with sodium pentobarbital (i.p., $50 \mathrm{mg} / \mathrm{kg}$ ). A state of areflexia was maintained throughout the surgery, and supplemental doses of dilute pentobarbital were administered sub-cutaneously if needed. Anesthesia level was monitored by response to toe pinch. Atropine and dexamethasone were administered sub-cutaneously to minimize secretions and brain edema during the procedure and recovery. Animals received antibiotic injections (ceftriaxone) before and after surgery. Body temperature was measured by a rectal probe and maintained at $37^{\circ} \mathrm{C}$. Two structural screws were used to anchor the implant over the left hemisphere. The 4-pin connector was held in place with dental acrylic. Although some implants remained firmly in place for five months, many implants were lost due to skull growth following implantation. Implanted rats

were housed singly when not in the enriched environment to eliminate the tendency of rats in small cages to damage implants by excessive grooming.

\section{Stimulus Presentation and Data Analysis}

Middle latency evoked potential data were collected once each week for 21 weeks from each rat while placed in a sound attenuated booth. Recordings were made during the dark cycle in both housing conditions, to encourage the rats to be as alert as possible, but the rats did spend some time sleeping. EEG recordings indicate rats were in slow wave sleep no 
more than $25 \%$ of the time. Overall, no differences in activity levels, exploration, time spent sleeping, or arousal levels were noted between enriched and standard rats during recording sessions. Stimuli included a $25 \mathrm{~ms}$ 9-kHz tone and a $100 \mathrm{~ms}$ noise burst (70 dB SPL with 3 $\mathrm{ms}$ rise and fall times). Tones and noises were presented from a speaker centered above the cage and randomly interleaved every 10 seconds. These sounds were not identical to any of the sounds heard in the enrichment cage. Since there was no specific overrepresentation of the exposed frequencies of the enriched A1 map when assayed using microelectrode mapping, we expected similar results in the evoked potential data (i.e. a non-specific increase in cortical response strength). Therefore, $9-\mathrm{kHz}$ was selected for the evoked potential study because it was not presented as part of the enriched environment. Thus, rats did not hear the 9-kHz tone or noise burst outside of the evoked potential recording sessions. Signals were amplified (10,000X) and displayed on an oscilloscope for monitoring (filtered $0.1-800 \mathrm{~Hz}$ ). Data acquisition computers collected 250 traces of each tone and 125 traces of each noise. Trials with excessive motion artifacts $(>0.1 \mathrm{mV})$ were discarded prior to analysis of the mean evoked potential.

The first two negative peaks in the evoked response are referred to as the N1 and N2 ( 40 and $140 \mathrm{~ms}$, respectively; Figure $2.5 \mathrm{~A}$ ). The first positive peak is referred to as P1 ( $75 \mathrm{~ms})$. The N1 potential in response to the tone was composed of two distinct sub-peaks, which we refer to as N1a and N1b ( 25 and 45 ms, respectively; Figure 5.6 B).

The sum of the absolute values of the evoked potential (30-100 ms after sound onset) was used to quantify the size of the auditory evoked response. To eliminate variability in evoked potential amplitude due to individual differences in electrode position and recording 
characteristics, the size of the evoked response for each week was divided by the size on week 1. Logarithm base 2 of this ratio was used so that -1 and +1 indicates 2 -fold decreases and increases, respectively. 


\section{RESULTS}

Neurophysiologic responses were recorded from rats housed either in standard laboratory conditions or in an enriched environment (See Methods, Figures 2.1 and 2.2). Action potentials were recorded from small groups of A1 neurons at more than 800 sites in 16 rats. Twenty-one rats were implanted with EEG electrodes and evoked responses were recorded from auditory cortex each week for up to 5 months. By combining these two techniques, we were able to document the effects of environmental enrichment over time and in fine spatial detail.

\section{Experiment 1 - Extracellular Recordings}

Although rats in the enriched environment were exposed to a variety of broadband and narrowband stimuli, environmental plasticity was quantified by recording A1 responses to simple tones and white noise bursts. Comparisons between responses from animals housed in enriched ( $\mathrm{n}=8$ rats; 462 sites) and standard ( $\mathrm{n}=8$ rats; 358 sites) conditions indicated that environment substantially altered response strength, receptive field size, intensity threshold, spontaneous rate, and response latency. Enrichment did not significantly alter the A1 map of tone frequency. In both groups, best frequency was highly correlated with anterior-posterior position $\left(r^{2}=0.83 \pm 0.02\right.$ and $r^{2}=0.82 \pm 0.03$ for enriched and standard $)$. The average change in best frequency as a function of anterior-posterior distance was not significantly different between the 2 groups $(-0.31 \pm 0.01$ and $-0.27 \pm 0.02$ for enriched and standard, in octaves/mm). In addition, we quantified the total area of A1. The total size of A1 was also unaffected by enrichment ( $1.03 \pm 0.17$ (enriched) $0.80 \pm 0.15 \mathrm{~mm}^{2}$ (standard), $\mathrm{p}>.05$ ). No 
overrepresentation of any frequency-intensity combination (including the $2.1,3$ or $4 \mathrm{kHz}$ frequencies used in the enriched condition) was observed (Kilgard and Merzenich, 1998).

The number of action potentials in response to tones or noises increased by one-third in enriched rats compared to rats housed in the standard condition (Table 2.1). A1 neurons in enriched rats were nearly $3 \mathrm{~dB}$ more sensitive to tones and were slightly more selective for tone frequency than neurons in the standard group (Table 2.1 and Figure 2.3). Bandwidth at 10-40 dB above threshold was narrower for enriched rats, but only reached statistical significance at $40 \mathrm{~dB}$ above threshold. The end of response was later as a result of the greater number of spikes evoked by each sound (Table 2.1 and Figure 2.4). In addition, spontaneous activity in enriched rats was $21 \%$ higher. Due to the greater rise in driven rate over spontaneous rate, the average signal to noise ratio increased by $11 \%$. 


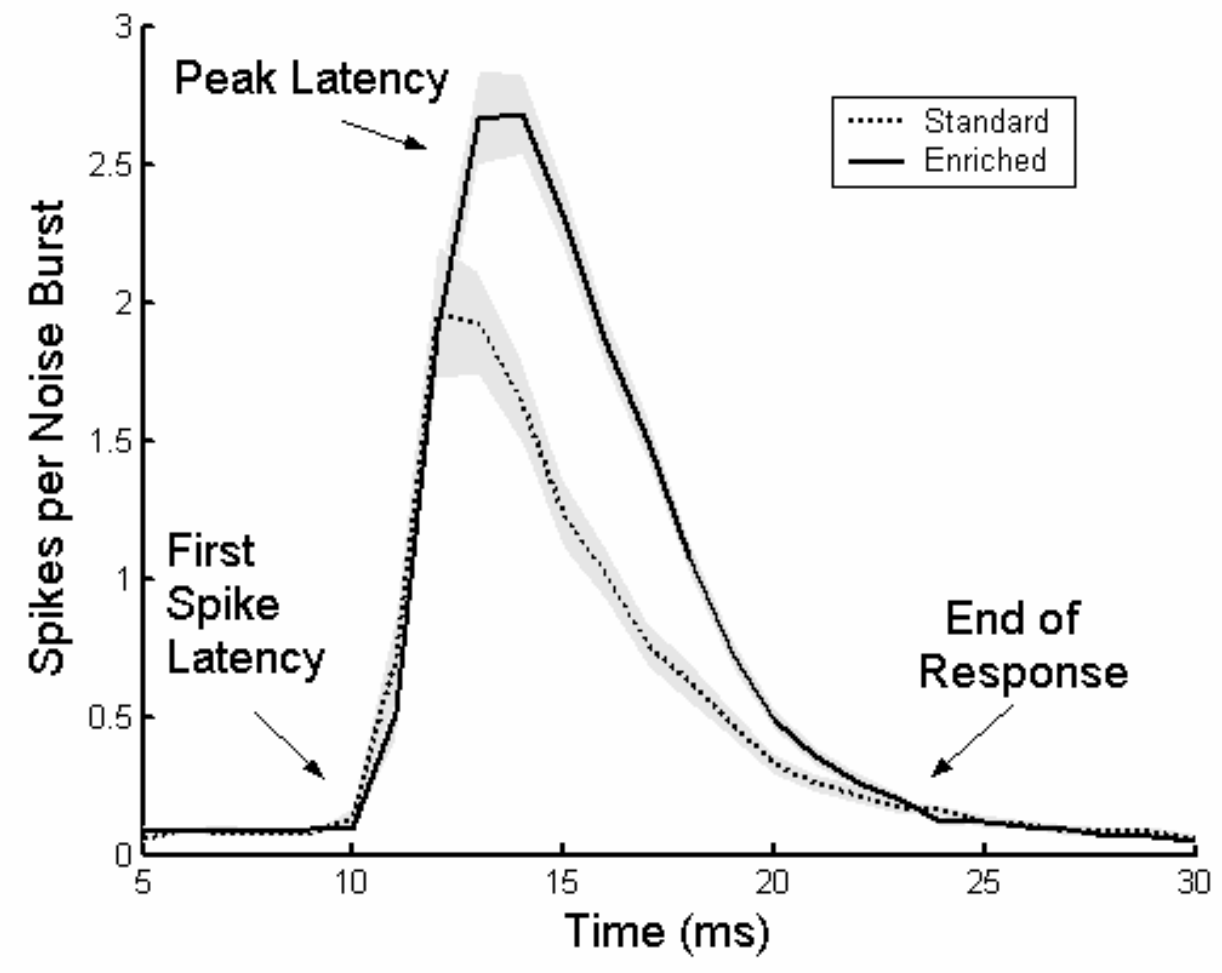

Figure 2.4. Mean noise burst PSTH's for enriched and standard rats ( $n=462,358$ sites). Enriched rats exhibit a higher firing rate, late peak and end of response latency. The gray shaded regions represent the standard error of the mean for each group. 


$\begin{array}{lccc}\text { RESPONSE PARAMETER } & \text { ENRICHED } & \text { STANDARD } & \text { P VALUE } \\ \text { Response Strength (spikes/tone) } & 1.61 \pm 0.04 & 1.24 \pm 0.06 & <0.00001 \\ \text { Response Strength (spikes/noise) } & 1.44 \pm 0.04 & 1.04 \pm 0.06 & <0.00001 \\ \text { Neural Threshold (dB) } & 17.19 \pm 0.47 & 19.88 \pm 0.61 & <0.001 \\ \text { Receptive Field Size (BW40) } & 2.01 \pm 0.04 & 2.16 \pm 0.05 & <0.05 \\ \text { Peak Latency (ms) } & 19.18 \pm 0.18 & 18.32 \pm 0.25 & <0.01 \\ \text { End of Peak Latency (ms) } & 36.09 \pm 0.39 & 34.41 \pm 0.40 & <0.01 \\ \text { Spontaneous (spikes / 20 msec) } & 0.114 \pm 0.004 & 0.094 \pm 0.006 & <0.01\end{array}$

Table 2.1. Response properties (mean \pm standard error) of primary auditory cortex neurons recorded from rats housed in enriched and standard conditions. Student's $t$-tests were used to determine statistical significance. 


\section{Experiment 2 - Auditory Evoked Potentials}

To document the effect of behavioral state and determine the time-course of environmental plasticity, auditory evoked potentials were recorded each week for 21 weeks from rats implanted with chronic epidural electrodes (Figure 2.2). After implantation, each 5-week old rat was randomly assigned to either the enriched or standard housing condition. Noise-evoked Responses

A $70 \mathrm{~dB}$ noise burst resulted in negativities $\sim 40$ and $\sim 140 \mathrm{~ms}$ after noise onset (N1 and N2) and positivity $\sim 75 \mathrm{~ms}$ after onset (P1) (Figure $2.5 \mathrm{~A}$ ). The shapes of the grand mean average evoked potentials from each group were similar during the first few weeks of differential housing (Figure 2.5 A-B). After 5 weeks, the average evoked potential amplitude from the enriched group was twice the size of the standard housing group (Figure 2.5 C). When the enriched group was transferred to the standard environment at 14 weeks of age, the amplitude of the auditory evoked potential was reduced by $60 \%$ within a week of moving (Figure 2.5 C, D). When the standard condition group was transferred to the enriched environment, the response amplitude doubled within a week (Figure 2.5 D, E). The noiseevoked responses of the early and late-enriched groups were indistinguishable when both were housed in the standard environment at weeks 9 and 18 (Figure 2.5 D, F).

Since responses of animals in the standard condition decreased over the first several weeks, while responses of enriched animals were unaltered, it appears that enrichment may protect cortical responses from decline rather than strengthening them (Figure 2.5 A-C). 
Alternatively, it may not be possible to increase the response to noise bursts since nearly all neurons in A1 already respond to broadband sounds. To avoid this potential ceiling effect, we also quantified the cortical response to a $9-\mathrm{kHz}$ tone $(70 \mathrm{~dB})$, which activates approximately half as many A1 neurons (Kilgard and Merzenich, 1999).

Tone-evoked Responses

As expected, responses to tones were significantly smaller than noise-evoked responses. Differences in tone-evoked response amplitude were evident after as little as 2 weeks of differential housing (Figure 2.6 A, B). The tone-evoked potentials from the standard group decreased by nearly 2 -fold over the first several weeks of the experiment (Figure 2.6 A, C). In contrast, the tone-evoked potential from the enriched group increased to nearly twice its initial peak-to-peak amplitude. Both P1 and N1 amplitudes were significantly larger for the early-enriched group compared to the group housed in the standard environment $(\mathrm{P} 1: 0.07 \mathrm{mV} \pm 0.01 \mathrm{mV}$ vs. $0.03 \mathrm{mV} \pm 0.01 \mathrm{mV}, \mathrm{p}<.05 ; \mathrm{N} 1:-0.05$ $\mathrm{mV}+-0.01 \mathrm{mV}$ vs. $-0.03 \mathrm{mV} \pm .01 \mathrm{mV}, \mathrm{p}<.05)$. Similar changes were also observed when the responses of individual animals were compared before and after enrichment. This paired analysis revealed a significant increase in amplitude for both $\mathrm{P} 1(0.03 \mathrm{mV} \pm 0.01 \mathrm{mV}$, $\mathrm{p}<.005)$ and $\mathrm{N} 1(-0.02 \mathrm{mV} \pm 0.01 \mathrm{mV}, \mathrm{p}<.05)$. The strengthening of the tone-evoked response occurred in both young rats and adult rats (Figure $2.6 \mathrm{C} \& \mathrm{E}$ ).

The N1 component of the tone-evoked response exhibited two distinct sub-peaks not seen in responses to noise. Most of the difference in N1 amplitude due to differential housing resulted from changes in the N1b (Figure 2.6 C). The N1b in enriched rats was 
nearly twice as large after 5 weeks compared to week 1, while the N1b in standard-housed rats was completely absent weeks 2-9 (Figure 2.6 B-D). The N1b developed within a week of moving standard-housed animals to the enriched environment (Figure 2.6 D-E). While the response amplitude from both groups was reduced when they were moved to the standard environment, the N1b was still evident many weeks later (Figure 2.6 F). These results indicate that some effects of enrichment may endure beyond the period of enrichment. However, most aspects of the auditory evoked response reflect environmental conditions during the prior week

Consistent with the observed increase in the N1b, the peak latency of the N1 was found to increase with enrichment (33.55 ms $\pm 2.58 \mathrm{~ms}$ vs. $23.44 \mathrm{~ms} \pm 0.83 \mathrm{~ms}, \mathrm{p}<.01)$. Similar changes were also observed when the latencies from individual animals were compared before and after enrichment $(7.70 \mathrm{~ms} \pm 3.30 \mathrm{~ms}, \mathrm{p}<.05)$. These results are consistent with data from the acute experiment in which strengthened responses exhibited later peak latencies. Interestingly, the latency of the P1 decreased in the enriched group compared to the standard-housed group $(69.73 \mathrm{~ms} \pm 1.88 \mathrm{~ms}$ vs. $88.22 \mathrm{~ms} \pm 8.93 \mathrm{~ms}, \mathrm{p}<.01)$. Although the neural generator of the $\mathrm{P} 1$ is not clear, it is possible that the decrease in $\mathrm{P} 1$ latency due to enrichment results from earlier activation of non-primary cortical fields. 


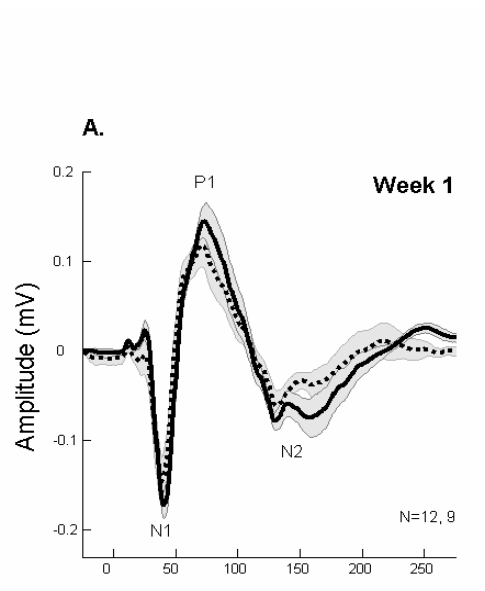

D.

B.
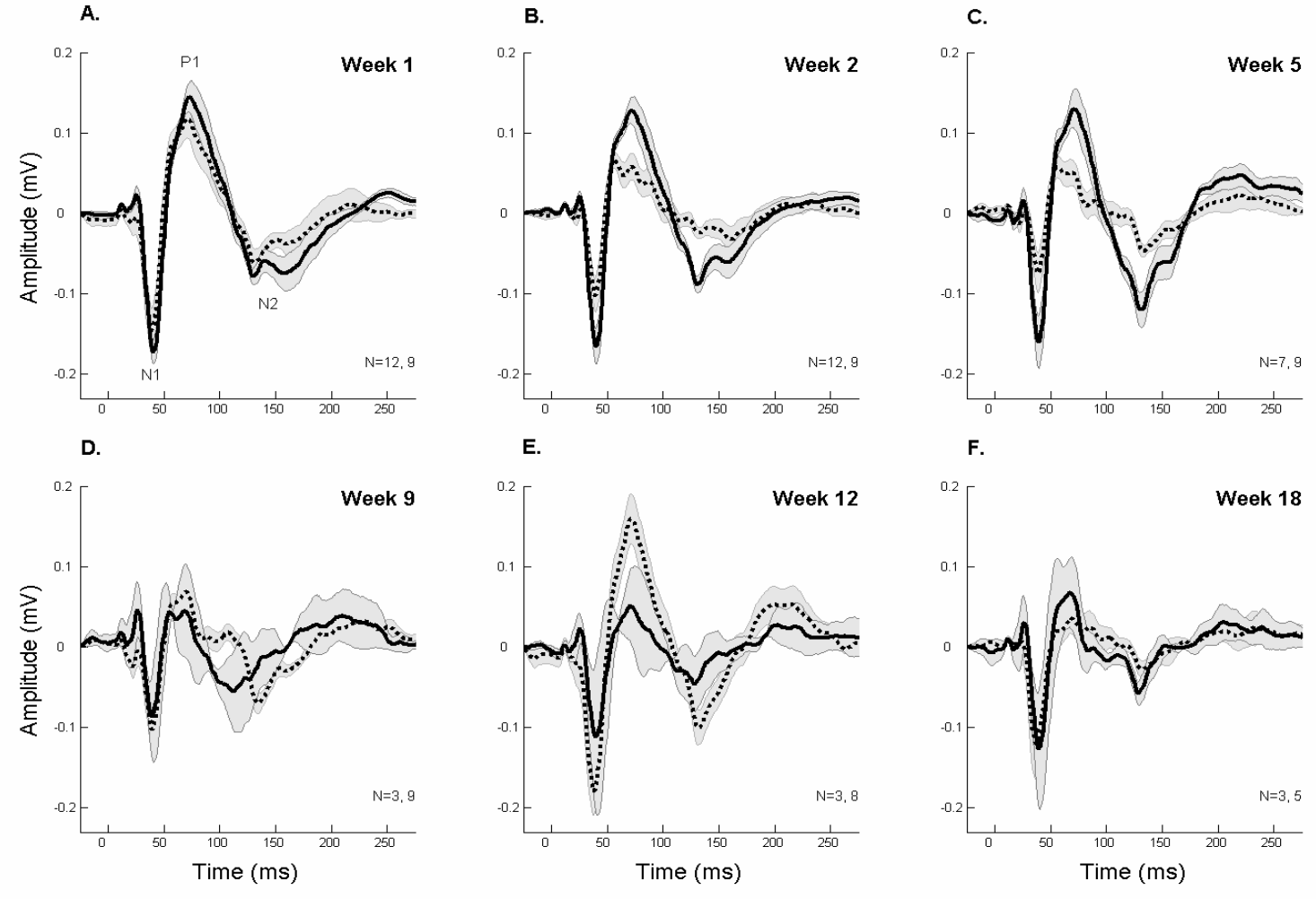

E.

F.
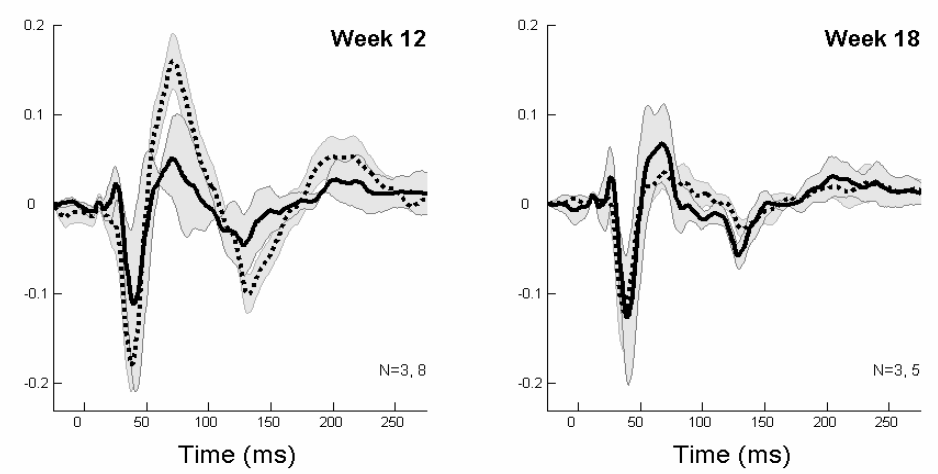

Figure 2.5. Grand mean average responses to noise for select weeks of the experiment. A) After 1 week of differential housing, auditory evoked potentials from the early-enriched group (Experiment 2a) were indistinguishable from the late-enriched group (Experiment 2b). $\mathrm{B}$ and $\mathrm{C}$ ) After several weeks of differential housing, responses of rats in the standard environment (dashed) were decreased compared to enriched rats (solid). D and F) Responses were once again indistinguishable when both groups were housed in the standard environment during weeks 9 and 18. E) After the housing conditions were switched, responses from the late-enriched group (dashed) increased and responses from the earlyenriched group (solid) decreased. The gray shaded regions represent the standard error of the mean for each group. 

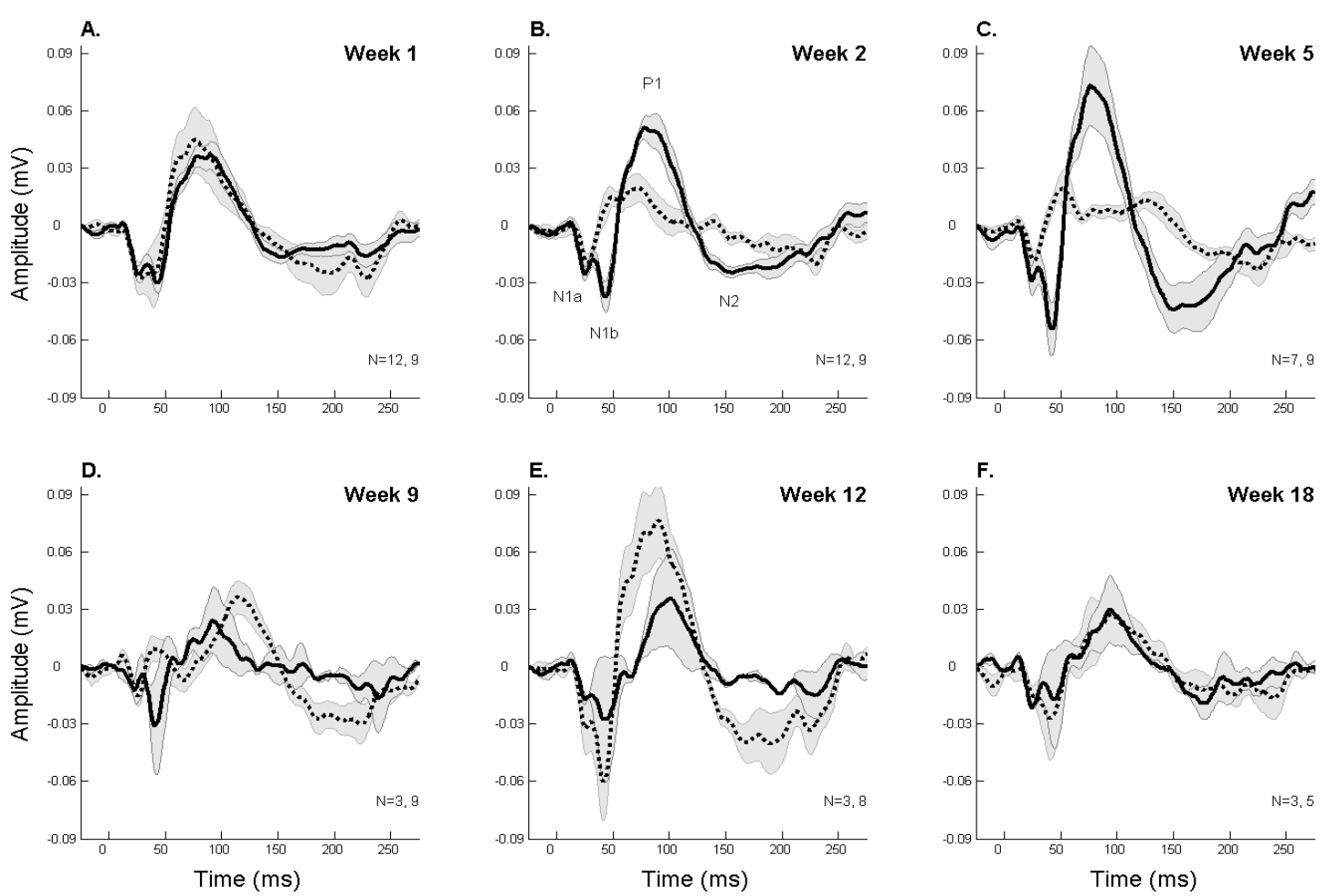

Figure 2.6. Grand mean average responses to $9-\mathrm{kHz}$ tone for selected weeks of the experiment. A) After 1 week of differential housing, auditory evoked potentials from the early-enriched group (Experiment 2a) were indistinguishable from the late-enriched group (Experiment 2b). B) After 2 weeks of differential housing, responses of rats in the enriched (solid) environment were larger than responses from rats in the standard (dashed) environment. C) By 5 weeks of differential housing, responses of enriched rats were three times the amplitude of standard-housed rats. D) Responses were diminished within 1 week after moving the early-enriched group to the standard housing condition. E) After moving to the enriched environment, the responses of the late-enriched group (dashed) increased to twice the amplitude of the early-enriched rats (solid). F) Tone responses were indistinguishable when both groups were housed in the standard environment during weeks 9 and 18. The gray shaded regions represent the standard error of the mean for each group. 
Time-course of Auditory-evoked Plasticity

Normalizing each individual's responses to their week 1 response eliminates variability due to electrode placement and facilitates direct comparison of tone and noise response plasticity. The relative changes in tone and noise-evoked responses over time for each experimental group are illustrated in Figure 2.7. A plasticity index value of 1 indicates a 2-fold increase in the magnitude of the evoked potential compared to week 1 , while a value of -1 indicates the magnitude of the evoked potential was halved (see Methods). While the average noise-evoked plasticity index for enriched and deprived rats was significantly different $(\mathrm{p}<0.05)$, the difference was only 0.57 during weeks $2-8$ and 0.83 during weeks 10-18 (Figure 2.8 A). The differences in the tone-evoked plasticity indices for enriched and deprived rats were larger: 1.21 during weeks 2-8 and 1.63 during weeks 10-18 (Figure 2.8 B). The larger influence of environment on tone-evoked responses may be due to a ceiling effect caused by the near maximal activation of auditory neurons by noise bursts. To confirm this observation, we also recorded responses from a quieter $(50 \mathrm{~dB})$ noise burst that generated responses that were intermediate between tones and loud noise bursts. The scale of environmental plasticity documented with a quiet noise burst was also intermediate to the plasticity observed using tones or loud noise bursts (.96 during weeks 2-8 and 1.49 during weeks 10-18). This finding indicates that stimuli selected to evoke the maximal response may not be optimal for documenting cortical plasticity. 

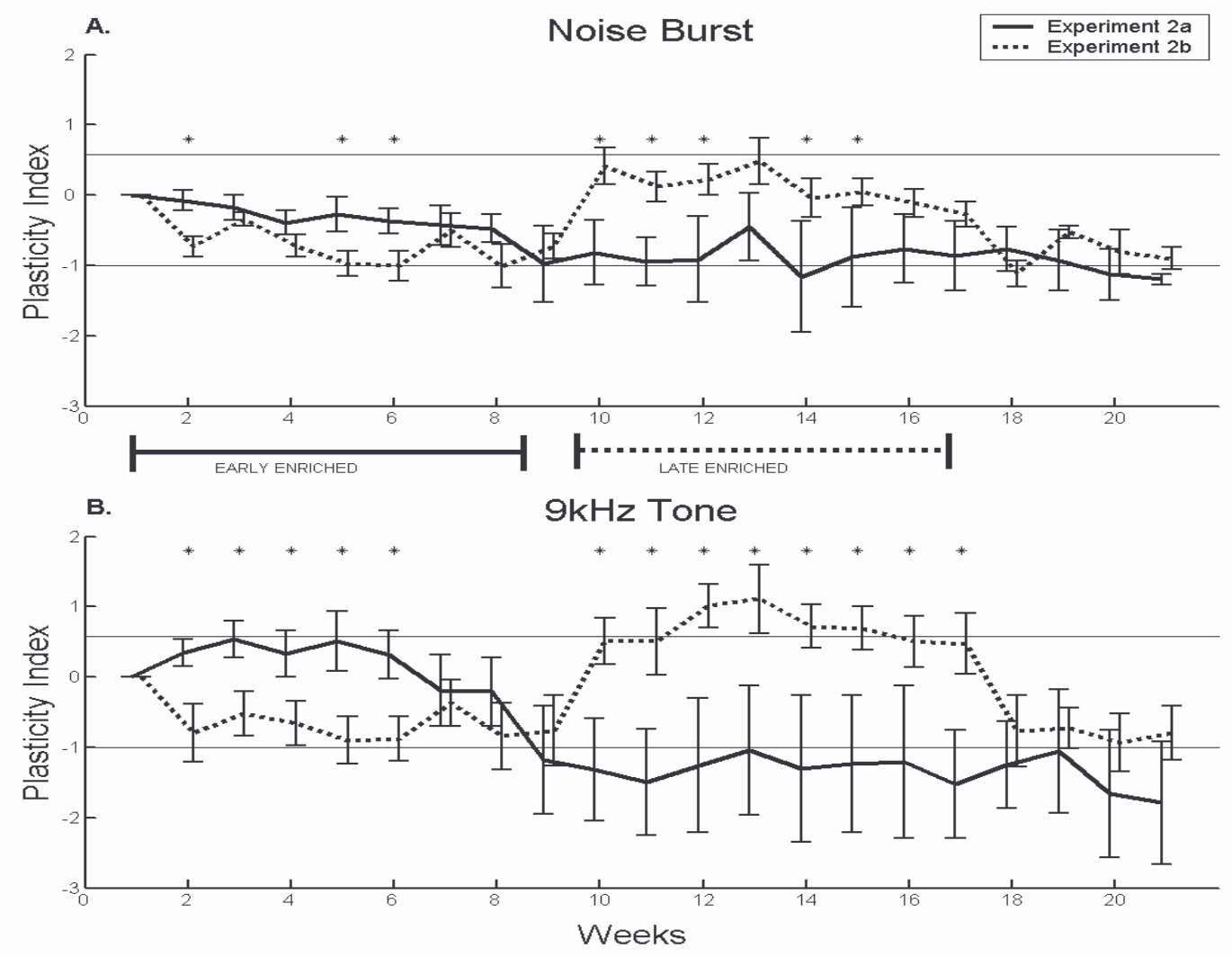

Figure 2.7. Time-course of environmental plasticity. Each rat's average evoked potential for each week was normalized to their response recorded on week 1 . The plasticity index represents the logarithm base 2 of this ratio, such that a value of 1 indicates a 2 -fold increase in response strength. A) The noise-evoked response was up to 2-fold larger in enriched compared to standard-housed rats. B) The tone-evoked response was up to 3-fold larger in enriched compared to standard-housed rats. Asterisks indicate significant differences between early- and late-enriched groups. Horizontal lines indicate increases and decreases of $50 \%$ compared to week 1. Periods of environmental enrichment for each group are indicated. Both groups were housed in the standard environment during experimental weeks 9 and 1921. The early-enriched group (solid) was in the enriched environment for 8 weeks and the late-enriched group (dashed) moved to the enriched environment after the recording on week 9. Both groups were 5 weeks old at the beginning of the experiment. Error bars represent standard error of the mean. 

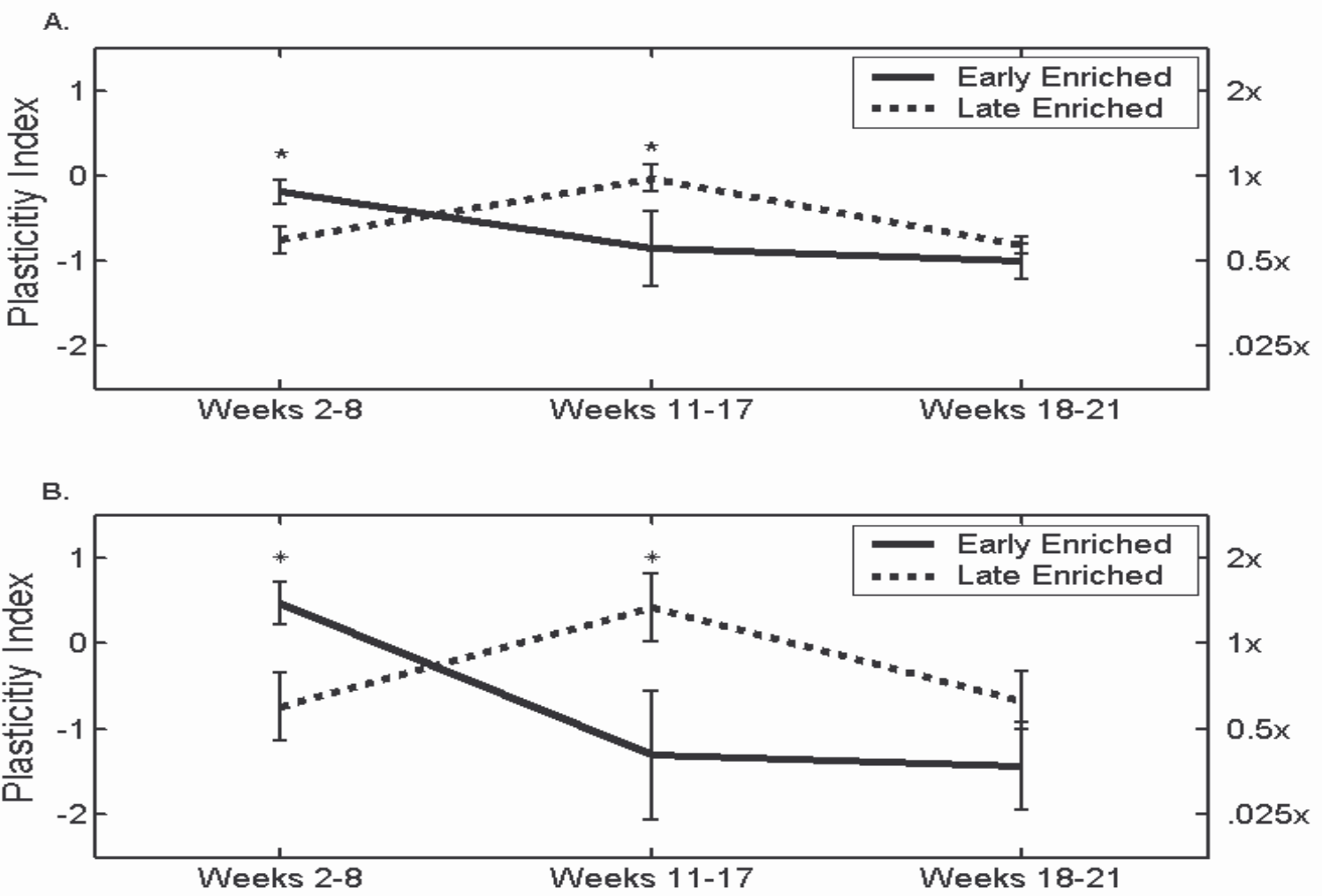

Figure 2.8. Housing condition significantly affects response strength of evoked potentials. The mean response evoked by both a noise (A) burst and tone (B) was significantly stronger while in the enriched environment compared to the standard housing condition. Environmental plasticity was equally strong in sexually immature ( $<13$ weeks old) and sexually mature (>13 weeks old) rats. 
Although the physiological effects of environmental enrichment were large (2-3 fold enhancement relative to standard housing), they typically did not last longer than 1 week after a return to the standard environment. In every case, the mean plasticity index significantly decreased when rats were moved from the enriched to the standard condition and significantly increased when rats were moved from the standard to the enriched condition (Figure 2.8). There were no significant differences in the scale of plasticity that occurred in young and adult rats (Figure 2.8). 


\section{DISCUSSION}

This study was designed to evaluate the neurophysiologic consequences of environmental enrichment on auditory cortex neurons. Microelectrode mapping provides the greatest spatial precision in documenting cortical plasticity. This technique made it possible to document improvements in A1 response strength, latency, threshold, and frequency selectivity; however, neural responses from each rat could only be sampled at one time point. Evoked potentials were also recorded from auditory cortex in awake rats to document the time-course of changes in response strength. Both methods confirmed that enrichment substantially enhances cortical responses. This environmental plasticity developed in less than 2 weeks of enrichment and reversed after 1 week in standard housing. These results indicate that auditory cortex neurons in young and adult rats are remarkably sensitive to environmental conditions.

While physiological plasticity studies in humans and animals often probe neural responses with intense stimuli, more reliable plasticity may be observed using stimuli that do not elicit saturated responses (Wible et al., 2002). In the present study, the greatest proportional changes in evoked response were recorded using sounds that stimulate less than the maximal neural response. A $9-\mathrm{kHz}$ tone generates an auditory evoked potential that is one-third the amplitude of a noise burst of the same intensity, because epidural recordings are influenced by neurons with a wide range of frequency preferences. Despite the better signal to noise ratio in recordings evoked by noise bursts, environmental plasticity was more reliable and proportionally larger when assayed using tones. Thus, it appears that stimuli 
which evoke smaller neural responses can avoid a potential ceiling effect and reveal a greater influence of enrichment on cortical responsiveness. To confirm this observation, we also recorded responses elicited by a quieter $(50 \mathrm{~dB})$ noise burst that generated responses that were intermediate between tones and loud noise bursts. The environmental plasticity documented using this sound was intermediate between the effects seen in Figures 2.5 and 2.6 (data not shown).

\section{Potential Mechanisms}

Changes in cellular, synaptic or network properties may contribute to the experiencedependent modifications induced by environmental conditions (Katz and Shatz, 1996; Gilbert, 1998; Syka, 2002). Acute sampling from A1 neurons in enriched rats revealed that increased response strength was accompanied by decreased response threshold and receptive field size. Smaller receptive fields were also observed in visual and somatosensory cortex after enrichment (Beaulieu and Cynader, 1990; Coq and Xerri, 1998).

The persistence of these effects under general anesthesia is consistent with the earlier conclusions that structural changes contribute to environmental plasticity. Reduced inhibition could explain the increased excitability and decreased threshold of A1 neurons in enriched rats. Enriched cats had $25 \%$ fewer GABAergic synapses in visual cortex than standard-housed cats (Beaulieu and Colonnier, 1987). Application of the GABA antagonist bicuculline increased response sensitivity, spontaneous activity, and maximum discharge rate in visual, somatosensory, and auditory cortex (Dykes et al., 1984; Eysel et al., 1998; Wang et al., 2000; Wang et al., 2002). Although GABAergic blockade mimics several of the effects 
of enrichment, bicuculline causes receptive fields to increase, rather than decrease in size. While it appears that decreased inhibition influences physiologic properties in enriched cortex, additional mechanisms are likely to contribute to environmental plasticity. Differences in other modulatory neurotransmitter levels may also affect response properties in enriched cortex. Enrichment increases levels of cortical norepinephrine, dopamine, acetylcholine, and acetylcholinesterase (Park et al., 1992; Feenstra et al., 1995; Giovannini et al., 2001; Naka et al., 2002). Interestingly, iontophoretic application of norepinephrine decreases receptive field size in auditory cortex neurons and acetylcholine decreases thresholds (Metherate et al., 1990; Manunta and Edeline, 1997). These results indicate that changes in multiple neurotransmitter systems could explain many of our physiological findings; however, structural changes are likely to contribute as well.

Our findings that enrichment-induced strengthening develops over many days and is maintained under general anesthesia, support earlier evidence of neuroanatomical changes induced by environmental enrichment. Although the effects of enrichment on dendritic length and branching and oligodendrocyte to neuron ratio persisted after several weeks of individual housing, the increase in cortical thickness reversed when rats were returned to standard housing conditions (Katz and Davies, 1984; Camel et al., 1986). Age-related changes in motor cortex such as broad receptive fields, small cortical maps and decreases in response strength were restored when aged rats were exposed to an enriched environment (Godde et al., 2002). Since the amplitude of the auditory evoked response reflected environmental conditions during the prior week, it appears that multiple factors with different time-courses contribute to environmental plasticity. 


\section{Remaining Questions}

Both epidural evoked potentials and microelectrode recordings demonstrate significant cortical plasticity. However, the proportional changes recorded with these techniques were markedly different. In awake rats, enrichment resulted in a greater than 2fold increase in the tone-evoked response, while in anesthetized rats the number of action potentials increased by only one-third. Three factors could be responsible for the greater enhancement of awake evoked potentials compared to anesthetized action potentials. First, anesthesia may eliminate possible neuromodulatory differences in awake standard and enriched rats. Second, evoked potentials may exhibit a greater capacity to increase because they are generated by summed synaptic potentials, while the action potentials recorded in the acute experiments are generated by a thresholding non-linearity and are thus more easily saturated. Finally, it is possible that non-primary auditory regions, which likely contribute to the evoked potentials, are more sensitive to environmental conditions than A1 (Eggermont and Ponton, 2002). The latency of the N1a peak ( 25ms) is similar to the peak response of A1 neurons recorded with microelectrodes. The greatest changes in the evoked potentials occurred considerably later ( $\mathrm{N} 1 \mathrm{~b}$ at $\sim 45 \mathrm{~ms}$ and the P1 at $\sim 75 \mathrm{~ms}$ ). These differences suggest that plasticity in auditory fields surrounding A1 may have had a substantial contribution to evoked potential plasticity. Future studies using chronically implanted microelectrode arrays to record from A1 and other auditory fields are needed to distinguish between these potential explanations. 
Previous studies have shown that multiple factors influence the degree of plasticity generated by environmental enrichment. These include physical activity, enrichment duration, social experience, behavioral relevance of sensory events, and age. For example, simple wheel running has been shown to increase cell proliferation and neurogenesis in the adult mouse dentate gyrus (van Praag et al., 1999). Even a few hours of daily enrichment increased brain weight, acetylcholinesterase staining, and RNA/DNA ratios (Ferchmin and Bennett, 1975; Will et al., 1977). While passive sensory enrichment failed to alter brain weight in rats, social interactions significantly increased brain weight (Ferchmin and Bennett, 1975). Studies in primates have shown that focused attention is required if sensory inputs are to stimulate cortical plasticity (Recanzone et al., 1993; Ahissar and Ahissar, 1994). These results indicate that both social interactions and attention contribute to the expression of cortical plasticity. The enriched environment used in this study was designed to expose rats to a wide variety of behaviorally meaningful sensory inputs. While background sounds were present in both environments, in the standard environment these sounds had little behavioral relevance and were less diverse than the sounds in the enriched environment. The enriched housing condition was also designed to increase the behavioral relevance by providing greater social interactions than the standard environment. However, this study cannot determine whether social experience, behavioral relevance of sensory events, attention, physical activity, or enrichment duration were important factors in altering cortical responses.

Exposure to behaviorally relevant sounds that are spectrally restricted (i.e. tones) can alter A1 topography, receptive field size and latency (Recanzone et al., 1993; Weinberger 
and Bakin, 1998). These changes are typically restricted to the region of the cortical map activated by these sounds. Although some tonal stimuli were part of the environmental enrichment, the plasticity effects documented in this study were not frequency-specific and were found across A1. Sensory sensitization due to random foot shock also strengthens responses across A1 (Bakin and Weinberger, 1990). However, these changes develop and fade much more quickly than the effects observed in this study and would likely not persist under the general anesthesia used in some of our experiments. Despite these differences, it remains likely that many of the same mechanisms involved in fear conditioning and perceptual learning are also involved in environmental plasticity.

Numerous studies have shown that the development of primary sensory cortex can be disrupted by abnormal sensory input in very young animals (Weliky and Katz, 1997; Zhang et al., 2002; Rema et al., 2003). For example, rats exposed to pulsed noise before 4 weeks of age exhibited disruption of A1 tonotopy and decreased frequency selectivity (Zhang et al., 2002), while pulsed noise exposure after 4 weeks of age resulted in no significant plasticity. Our results indicate that cortical responses can be degraded or enhanced in animals well beyond these early sensitive periods. This result is consistent with earlier observations that highly focused behavioral training can improve cortical responses in adults. Since the present study was not designed to determine how specific factors influence environmental plasticity, additional studies will be needed to evaluate the potentially interacting factors responsible for the profound physiological changes observed in this study. 


\section{Clinical Implications}

The results of this and earlier studies indicate that rich and stimulating environments can significantly improve the sensory information processing of cortical neurons. Although the exact consequences of plasticity on cortical development or recovery from injury are not clear, numerous studies suggest that environmental enrichment may be useful in promoting recovery from neurological disability (Biernaskie and Corbett, 2001). The decreased responsiveness of auditory cortex in congenitally deaf cats can be reversed by behaviorally meaningful electrical activation of the cochlea (Klinke et al., 1999). Plasticity that likely contributes to improvements in sensory and language function has also been observed in deaf patients following cochlear implantation (Sharma et al., 2002a, b). Exposure to an enriched environment significantly improved cortical circuitry in animal models of traumatic brain injury and may improve behavioral recovery in humans (Jones and Schallert, 1994; Nudo and Friel, 1999; Risedal et al., 2002). Focused and intensive environmental enrichment may ameliorate the sensory-perceptual and social abnormalities observed in individuals with autistic spectrum and other neurologic disorders (Merzenich et al., 1996; Baranek, 2002). Collectively, these studies suggest that neural plasticity mechanisms underlie much of the functional improvements resulting from rehabilitation. 


\section{REFERENCES}

Ahissar, E. and Ahissar, M. (1994). Plasticity in auditory cortical circuitry. Curr Opin Neurobiol, 4: 580-7.

Bakin, J. and Weinberger, N. (1990). Classical conditioning induces CS-specific receptive field plasticity in the auditory cortex of the guinea pig. Brain Res, 536(1-2):271-86

Baranek, G.T. (2002). Efficacy of sensory and motor interventions for children with autism. $J$ Autism Dev Disord, 32: 397-422.

Beaulieu, C. and Colonnier, M. (1987). Effect of the richness of the environment on the cat visual cortex. J Comp Neurol, 266: 478-94.

Beaulieu, C. and Cynader, M. (1990). Effect of the richness of the environment on neurons in cat visual cortex. I. Receptive field properties. Brain Res Dev Brain Res, 53: 71-81.

Bennett, E.L., Diamond, M.C., Morimoto, H. and Hebert, M. (1966). Acetylcholinesterase activity and weight measures in fifteen brain areas from six lines of rats. $J$ Neurochem, 13: 563-72.

Biernaskie, J and Corbett, D. (2001). Enriched rehabilitative training promotes improved forelimb motor function and enhanced dendritic growth after focal ischemic injury. $J$ Neurosci,2001;21(14):5272-80.

Camel, J.E., Withers, G.S. and Greenough, W.T. (1986). Persistence of visual cortex dendritic alterations induced by post-weaning exposure to a "super-enriched" environment in rats. Behav Neurosci, 100: 810-3.

Coq, J.O. and Xerri, C. (1998). Environmental enrichment alters organizational features of the forepaw representation in the primary somatosensory cortex of adult rats. Exp Brain Res, 121: 191-204.

Diamond, M.C. (2001). Response of the brain to enrichment. An Acad Bras Cienc, 73: 211-20. 
Diamond, M.C., Law, F., Rhodes, H., Lindner, B., Rosenzweig, M.R., Krech, D. and Bennett, E.L. (1966). Increases in cortical depth and glia numbers in rats subjected to enriched environment. J Comp Neurol, 128: 117-26.

Diamond, M.C., Rosenzweig, M.R., Bennett, E.L., Lindner, B. and Lyon, L. (1972). Effects of environmental enrichment and impoverishment on rat cerebral cortex. J Neurobiol, 3: 47-64.

Dykes, R.W., Landry, P., Metherate, R. and Hicks, T.P. (1984). Functional role of GABA in cat primary somatosensory cortex: shaping receptive fields of cortical neurons. $J$ Neurophysiol, 52: 1066-93.

Edeline, J.M. (1999). Learning-induced physiological plasticity in the thalamo-cortical sensory systems: a critical evaluation of receptive field plasticity, map changes and their potential mechanisms. Prog Neurobiol, 57: 165-224.

Eggermont, J.J. and Ponton, C.W. (2002). The neurophysiology of auditory perception: from single units to evoked potentials. Audiol Neurootol, 7: 71-99.

Eysel, U.T., Shevelev, I.A., Lazareva, N.A. and Sharaev, G.A. (1998). Orientation tuning and receptive field structure in cat striate neurons during local blockade of intracortical inhibition. Neurosci, 84: 25-36.

Feenstra, M.G., Botterblom, M.H. and van Uum, J.F. (1995). Novelty-induced increase in dopamine release in the rat prefrontal cortex in vivo: inhibition by diazepam. Neurosci Lett, 189: 81-4.

Ferchmin, P.A. and Bennett, E.L. (1975). Direct contact with enriched environment is required to alter cerebral weights in rats. J Comp Physiol Psychol, 88: 360-7.

Gilbert, C.D. (1998). Adult cortical dynamics. Physiol Rev, 78: 467-85.

Giovannini, M.G., Rakovska, A., Benton, R.S., Pazzagli, M., Bianchi, L. and Pepeu, G. (2001). Effects of novelty and habituation on acetylcholine, GABA, and glutamate release from the frontal cortex and hippocampus of freely moving rats. Neurosci 106: 43-53. 
Globus, A., Rosenzweig, M.R., Bennett, E.L. and Diamond, M.C. (1973). Effects of differential experience on dendritic spine counts in rat cerebral cortex. J Comp Physiol Psychol, 82: 175-81.

Godde B., Berkefeld T., David-Jurgens M., Dinse HR. (2002). Age-related changes in primary somatosensory cortex of rats: evidence for parallel degenerative and plasticadaptive processes. Neurosci Biobehav Rev, 26(7):743-52.

Greenough, W.T., Volkmar, F.R. and Juraska, J.M. (1973). Effects of rearing complexity on dendritic branching in frontolateral and temporal cortex of the rat. Exp Neurol, 41: 371-8.

Hilbig, H., Bidmon, H.J., Steingruber, S., Reinke, H. and Dinse, H.R. (2002). Enriched environmental conditions reverse age-dependent gliosis and losses of neurofilaments and extracellular matrix components but do not alter lipofuscin accumulation in the hindlimb area of the aging rat brain. J Chem Neuroanat, 23: 199-209.

Jones, T.A. and Schallert, T. (1994). Use-dependent growth of pyramidal neurons after neocortical damage. J Neurosci, 14: 2140-52.

Katz, H.B. and Davies, C.A. (1984). Effects of differential environments on the cerebral anatomy of rats as a function of previous and subsequent housing conditions. Exp Neurol, 83: 274-87.

Katz, L.C. and Shatz, C.J. (1996). Synaptic activity and the construction of cortical circuits. Science, 274: 1133-8.

Kilgard, M.P. and Merzenich, M.M. (1999). Distributed representation of spectral and temporal information in rat primary auditory cortex. Hear Res, 134:16-28.

Kilgard, M.P, Pandya, P.K., Vazquez, J.L., Gehi, A., Schreiner, C.E. and Merzenich, M.M. (2001). Sensory input directs spatial and temporal plasticity in primary auditory cortex, J Neurophys 86: 339-353.

Klinke, R., Kral, A., Heid, S., Tillein, J. and Hartmann, R. (1999). Recruitment of the auditory cortex in congenitally deaf cats by long- term cochlear electrostimulation. Science, 285:1729-33. 
Manunta, Y. and Edeline, J.M. (1997). Effects of noradrenaline on frequency tuning of rat auditory cortex neurons. Eur J Neurosci, 9: 833-47.

Merzenich, M., Wright B., Jenkins W., Xerri C., Byl, N., Miller, S., and Tallal, P. (1996). Cortical plasticity underlying perceptual, motor, and cognitive skill development: implications for neurorehabilitation. Cold Spring Harb Symp Quant Biol, 61: 1-8.

Metherate, R., Ashe, J.H. and Weinberger, N.M. (1990). Acetylcholine modifies neuronal acoustic rate-level functions in guinea pig auditory cortex by an action at muscarinic receptors. Synapse, 6: 364-8.

Naka, F., Shiga, T., Yaguchi, M. and Okado, N. (2002). An enriched environment increases noradrenaline concentration in the mouse brain. Brain Res, 924: 124-6.

Nudo, R.J. and Friel, K.M. (1999). Cortical plasticity after stroke: implications for rehabilitation. Rev Neurol, 155: 713-7.

Park, G.A., Pappas, B.A., Murtha, S.M. and Ally, A. (1992). Enriched environment primes forebrain choline acetyltransferase activity to respond to learning experience. Neurosci Lett, 143: 259-62.

Rampon, C., Jiang, C.H., Dong, H., Tang, Y.P., Lockhart, D.J., Schultz, P.G., Tsien, J.Z. and $\mathrm{Hu}, \mathrm{Y}$. (2000). Effects of environmental enrichment on gene expression in the brain. PNAS 97: 12880-4.

Recanzone, G.H., Schreiner, C.E. and Merzenich, M.M. (1993). Plasticity in the frequency representation of primary auditory cortex following discrimination training in adult owl monkeys. J Neurosci, 13: 87-103.

Rema V, Armstrong-James M, Ebner FF. (2003). Experience-dependent plasticity is impaired in adult rat barrel cortex after whiskers are unused in early postnatal life. $J$ Neurosci, 23(1):358-66.

Risedal, A., Mattsson, B., Dahlqvist, P., Nordborg, C., Olsson, T. and Johansson, B.B. (2002). Environmental influences on functional outcome after a cortical infarct in the rat. Brain Res Bull, 58: 315-21. 
Schapiro, S. and Vukovich, K.R. (1970). Early experience effects upon cortical dendrites: a proposed model for development. Science, 167: 292-4.

Sharma, A., Dorman, M.F. and Spahr, A.J. (2002a). Rapid development of cortical auditory evoked potentials after early cochlear implantation. Neuroreport, 13: 1365-8.

Sharma, A., Dorman, M.F. and Spahr, A.J. (2002b). A sensitive period for the development of the central auditory system in children with cochlear implants: implications for age of implantation. Ear Hear, 23: 532-39.

Sirevaag, A.M. and Greenough, W.T. (1987). Differential rearing effects on rat visual cortex synapses. III. Neuronal and glial nuclei, boutons, dendrites, and capillaries. Brain Res, 424: 320-32.

Staiger, J.F., Masanneck, C., Bisler, S., Schleicher, A., Zuschratter, W. and Zilles, K. (2002). Excitatory and inhibitory neurons express c-Fos in barrel-related columns after exploration of a novel environment. Neurosci, 109: 687-99.

Syka, J. (2002). Plastic changes in the central auditory system after hearing loss, restoration of function, and during learning. Physiol Rev, 82: 601-36.

van Praag, H., Kempermann, G. and Gage, F.H. (2000). Neural consequences of environmental enrichment. Nat Rev Neurosci, 1: 191-8.

van Praag, H., Kempermann, G. and Gage, F.H. (1999). Running increases cell proliferation and neurogenesis in the adult mouse dentate gyrus. Nat Neurosci, 2: 266-70.

Volkmar, F.R. and Greenough, W.T. (1972). Rearing complexity affects branching of dendrites in the visual cortex of the rat. Science, 176: 1145-7.

Wang, J., Caspary, D. and Salvi, R.J. (2000). GABA-A antagonist causes dramatic expansion of tuning in primary auditory cortex. Neuroreport, 11: 1137-40.

Wang, J., McFadden, S.L., Caspary, D. and Salvi, R. (2002). Gamma-aminobutyric acid circuits shape response properties of auditory cortex neurons. Brain Res, 944: 219-31. 
Weinberger, N.M. and Bakin, J.S. (1998). Learning-induced physiological memory in adult primary auditory cortex: receptive field plasticity, model, and mechanisms. Audiol Neurootol, 3: 145-67.

Weliky, M. and Katz, L.C. (1997). Disruption of orientation tuning in visual cortex by artificially correlated neuronal activity. Nature, 386: 680-5.

Wible, B., Nicol, T. and Kraus, N. (2002). Abnormal neural encoding of repeated speech stimuli in noise in children with learning problems. Clin Neurophysiol, 113: 485-94.

Will, B.E., Rosenzweig, M.R., Bennett, E.L., Hebert, M. and Morimoto, H. (1977). Relatively brief environmental enrichment aids recovery of learning capacity and alters brain measures after postweaning brain lesions in rats. J Comp Physiol Psychol, 91: 33-50.

Zhang, L.I., Bao, S. and Merzenich, M.M. (2002). Disruption of primary auditory cortex by synchronous auditory inputs during a critical period. PNAS 99: 2309-14. 


\title{
CHAPTER 3
}

\section{ENVIRONMENTAL ENRICHMENT INCREASES PAIRED-PULSE DEPRESSION IN RAT AUDITORY CORTEX}

\author{
Cherie R. Percaccio *, Navzer D. Engineer*, Autumn L. Pruette, Pritesh K. Pandya, \\ Raluca Moucha, Daniel L. Rathbun, Michael P. Kilgard \\ * These authors contributed equally to this work
}

School of Behavioral and Brain Sciences, GR41

The University of Texas at Dallas

P.O. Box 830688

Richardson, Texas 75083

Keywords: activity-dependent, long-term plasticity, paired-pulse facilitation, sensory gating, synaptic plasticity 


\section{ACKNOWLEDGEMENTS}

Navzer D. Engineer is the primary author of Experiment 1. Pritesh K. Pandya, Raluca

Moucha, Daniel L. Rathbun, and Michael P. Kilgard assisted Navzer with surgeries, data

collection, and analysis. Cherie R. Percaccio is the primary author of Experiment 2. Autumn

L. Pruette and Michael P. Kilgard assisted Cherie with analysis. The authors thank Wei Wei

Dai, Juliann Record, and Vikram Jakkamsetti for assistance with recording sessions and

animal colony management. This work was supported by the Cure Autism Now Foundation and the Callier Excellence in Education Fund. 


\section{ABSTRACT}

Temporal features are important for the identification of natural sounds. Earlier studies have shown that cortical processing of temporal information can be altered by long-term experience with modulated sounds (Kilgard and Merzenich, 1998; Bao et al., 2004). In a previous study we observed that environmental enrichment dramatically increased the response of cortical neurons to single tone and noise burst stimuli in both awake and anesthetized rats (Engineer et al., 2004). Here, we evaluate how enrichment influences temporal information processing in the auditory cortex. We recorded responses to repeated tones and noise bursts in awake rats using epidural evoked potentials and in anesthetized rats using microelectrodes. Enrichment increased the response of cortical neurons to stimuli presented at slow rates and decreased the response to stimuli presented at fast rates relative to controls. Our observation that enrichment substantially increased response strength and forward masking is consistent with earlier reports that long-term potentiation of cortical synapses is associated with increased paired-pulse depression (Markram and Tsodyks, 1996). Enrichment also increased response synchronization at slow rates and decreased synchronization at fast rates. Paired-pulse depression increased within days of environmental enrichment and was restored to normal levels after return to standard housing conditions. These results are relevant to several clinical disorders characterized by abnormal gating of sensory information, including autism, schizophrenia and dyslexia. 


\section{INTRODUCTION}

Temporal information is essential for identifying many natural sounds, including animal vocalizations and speech. Auditory cortex is involved in the perception of temporal distinctions (Merzenich et al., 1993). Humans, non-human primates, and rodents exhibit temporal processing deficits after auditory cortex lesions (Fitch et al., 1994; Herman et al., 1997; Mummery et al., 1999; Clark et al., 2000a, b; Harrington et al., 2001).

Sensory enrichment has been suggested as a treatment for temporal processing deficits found in some central auditory processing disorders. Training improves cortical processing of temporal information in humans and animals (Recanzone et al., 1992;

Merzenich et al., 1996; Tallal et al., 1996; Nagarajan et al., 1998; Tremblay et al., 2001; Beitel et al., 2003; Hayes et al., 2003; Warrier et al., 2004). Collectively, these results indicate that cortical temporal information processing can be altered by an intense schedule of exposure to modulated stimuli.

The experiments reported here document the effects of sensory enrichment on the processing of temporally modulated acoustic stimuli. An earlier study reported that environmental enrichment increased the preferred temporal frequency of cat primary visual cortex neurons (Beaulieu and Cynader, 1990). To test whether enrichment would increase or decrease the preferred modulation frequency of auditory cortex neurons, we collected extracellular recordings from anesthetized rats and surface evoked potentials from unanaesthetized rats housed in standard and enriched conditions. 


\section{MATERIAL AND METHODS}

All protocols and recording procedures conformed to the Ethical Treatment of Animals (NIH) and were approved by the committee on Animal Research at the University of Texas at Dallas.

\section{Environmental Conditions}

Thirty-five female Sprague-Dawley rats were used in this study. Rats in both housing conditions were on a reverse 12-hr light/dark cycle and heard the sounds of room traffic, feeding, and cleaning while they were most active. Constant temperature and humidity were maintained and food and water were provided ad libitum for all rats. All the animals used in this study were housed with their mothers and littermates until weaning at 4 weeks of age. The environmental conditions are identical to those described in our previous study (Engineer et al., 2004).

The standard housing condition consisted of 1-2 rats per cage (26 L x $18 \mathrm{~W}$ x $18 \mathrm{H}$ $\mathrm{cm}$, Figure 3.1 A). The acoustic environment of this condition included vocalizations from 20-30 other rats housed in the same room. In the enriched environment, 4-8 rats were housed together in a single large cage in a separate room from the main rat colony (Figure 3.1 B). Since rats reach sexual maturation between 8-12 weeks of age, after one month in the enriched environment, a vasectomized male rat was introduced into the cage to encourage natural social interactions.

Animals in the enriched environment were housed in a large cage (76 L x $45 \mathrm{~W}$ x 90 $\mathrm{H} \mathrm{cm}$ ) with four levels connected by ramps. Touch plates at the bottom of two ramps 
triggered different tones $(2100$ or $4000 \mathrm{~Hz}$ ) when rats stepped on the plates. Chains, wind chimes, and bells were hung across the entrance of each ramp so that a unique sound was elicited when rats passed from one level to the next. A motion detector emitted an electronic chime each time a rat crossed the infrared beam in front of the water source. An exercise wheel emitted a tone $(3000 \mathrm{~Hz}$ Piezo Speaker) and activated a small green light emitting diode with each rotation. Each movement-triggered sound had unique spectral and temporal characteristics that provided behaviorally meaningful information about the location and activity of other rats in the cage.

A CD player presented randomly selected sounds every 2 to 60 seconds, including simple tones, amplitude modulated and frequency modulated tones, noise bursts and other complex sounds (rat vocalizations, classical music, rustling leaves, etc.). Seven of the seventy-four sounds activated a pellet dispenser (Med Associates) that delivered a sugar pellet reward to encourage attention to the sounds. The rewarded tracks included interleaved tones of different carrier frequencies ( $25 \mathrm{~ms}$ long 4, 5, 9, 12, 14, and 19-kHz tones with interstimulus intervals ranging from $50 \mathrm{~ms}$ to $2 \mathrm{sec}$ ) and frequency modulated sweeps (one octave up or down in a 140 or $300 \mathrm{~ms}$ sweep with interstimulus intervals ranging from 80 to $800 \mathrm{~ms})$.

The power spectrums of sounds in the enriched environment spanned the entire hearing range of the rat (1-45 kHz), and were less than $75 \mathrm{~dB}$ SPL. The sound sources added to the enriched environment were provided $24 \mathrm{hrs}$ a day, and were designed to be more diverse and to provide more behaviorally relevant information than sounds in the standard condition. The average interval between the components of complex sounds in the enriched 
environment was significantly shorter compared to the standard environment (108 \pm 73 vs. $133 \pm 80 \mathrm{~ms}$, mean $\pm \mathrm{std}, \mathrm{p}<.001)$. Our definition of a complex sound was any sound louder than $50 \mathrm{~dB}$ SPL with more than one onset separated by no more than $300 \mathrm{msec}$. The total number of sounds ( $>50 \mathrm{~dB}$ SPL) was not significantly different between the enriched and standard environments ( $23 \pm 23$ vs. $19 \pm 15$ sound onsets per minute, mean \pm std).

Although it is possible that the enriched environment was mildly stressful, we observed no evidence of distress in any of the rats at any time (i.e. excessive licking, hair loss, fighting, etc.). Rats actively explored the environment, voluntarily activated sound sources, and engaged in playful behavior. Additionally, our behavioral observations and EEG data indicate that enrichment did not interfere with normal sleep-wake behavior. 
A. STANDARD ENVIRONMENT

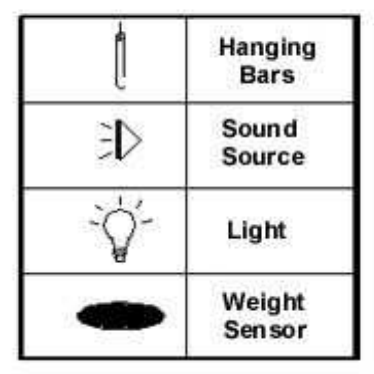

\section{B. ENRICHED ENVIRONMENT}

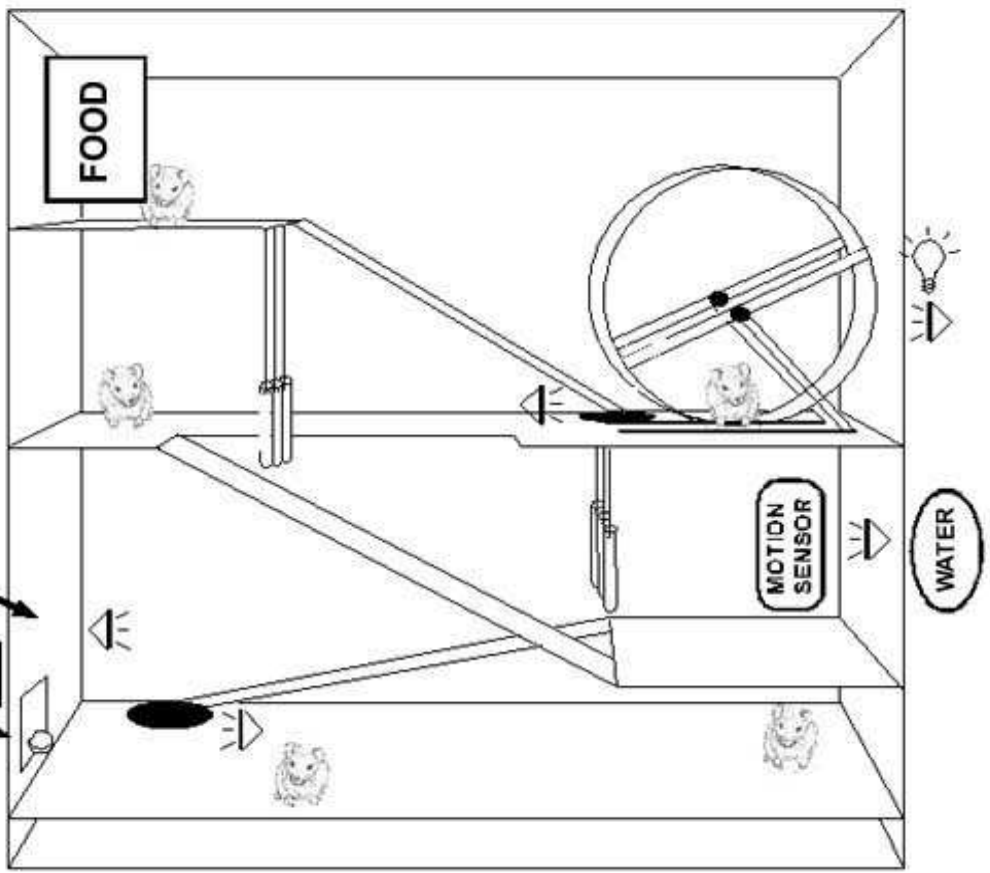

Figure 3.1. Schematic of standard and enriched housing conditions. A) The standard environment consisted of 1 or 2 rats housed in hanging cages within an animal colony room. B) The enriched environment consisted of 4-8 rats housed in a large cage with devices that generated different sounds when rats crossed a motion detector path, stepped on weight sensors, or passed through hanging bars. In addition, each rotation of the running wheel triggered a brief tone and light flash. A CD player played 74 sounds, including tones, noise bursts, musical sequences and other complex sounds in random order. Some of these sounds were associated with delivery of a sugar reward. While the sounds in the enriched environment were more diverse and behaviorally relevant, rats in both conditions heard approximately the same number of sounds each day. 


\section{Experiment 1 - Extracellular Recordings}

Thirty-day-old female Sprague-Dawley rats were randomly assigned to either the enriched environment $(n=8)$ or the standard condition $(n=6)$. The enriched rats were raised 45 per cage (in two sessions). Rats in the standard condition were raised 2 per cage. Acute microelectrode mapping was performed after 8 weeks in each environment (Figure 3.2 A, B). Although acute experiments were interspersed, it should be noted that the experimenters were not blind to the identity of the animal because of the unkempt state of the rats' fur typical of enriched animals. However, the sampling density and depth of recordings made in enriched and standard-housed rats were indistinguishable and all analysis was performed blind to housing condition.

Acute Surgery

Surgical anesthesia was induced with sodium pentobarbital (50 mg/kg, i.p.). A state of areflexia was maintained throughout the surgery and recording phases with supplemental doses of dilute pentobarbital $(0.2-0.5 \mathrm{ml} ; 8 \mathrm{mg} / \mathrm{ml}$, i.p.). The interval between supplements varied depending on the anesthetic state of the animal, but was typically every 1-1.5 hrs. Anesthesia depth was evaluated by heart rate, breathing rate, corneal reflexes and response to toe pinch. These indicators were indistinguishable between the two groups. Circulatory function was monitored with EKG and pulse oximetry. Fluid balance was maintained with a 1:1 mixture of $5 \%$ dextrose and Ringer's lactate $(\sim 0.5 \mathrm{ml} / \mathrm{hr})$. Body temperature was maintained at $37^{\circ} \mathrm{C}$. The trachea was cannulated to ensure adequate ventilation and minimize breathing sounds. Humidified air was delivered to the open end of 
the cannula. After the cisterna magnum was drained to minimize cerebral edema, the right auditory cortex was exposed and the dura removed. Since previous studies implicated the importance of the right hemisphere in processing temporally modulated sounds, this hemisphere was selected for recording responses to complex tone trains (Wetzel et al., 1998).

The cortex was maintained under a layer of viscous silicon oil to prevent desiccation during the 24-30 hr experiment. Penetration locations were referenced using cortical vasculature as landmarks. A detailed map of auditory cortex was generated from 50-100 microelectrode penetrations. A pair of parylene coated tungsten microelectrodes (FHC, 250 $\mu \mathrm{m}$ separation, $2 \mathrm{M} \Omega$ ) was lowered $550 \mu \mathrm{m}$ below the pial surface (layer 4/5) of the auditory cortex. Action potentials from a small cluster of neurons were collected at each penetration site.

Action potentials were recorded simultaneously from two tungsten microelectrodes. The neural signals were filtered $(0.3-15 \mathrm{kHz})$ and amplified $(10,000 \mathrm{X})$. As in our earlier experiment, potentials above approximately $0.18 \mathrm{mV}$ were considered to be action potentials (Pandya et al., 2005). The borders of A1 were defined based on continuous topography of characteristic frequency (CF) and short response latency. Sites with high thresholds, long latencies, broad tuning and discontinuities in $\mathrm{CF}$ topography were considered non-A1 and excluded from further analysis (Kilgard et al., 2001). Criteria for identifying non-A1 sites was subjectively applied by well-trained blind observers. 
Age (weeks)

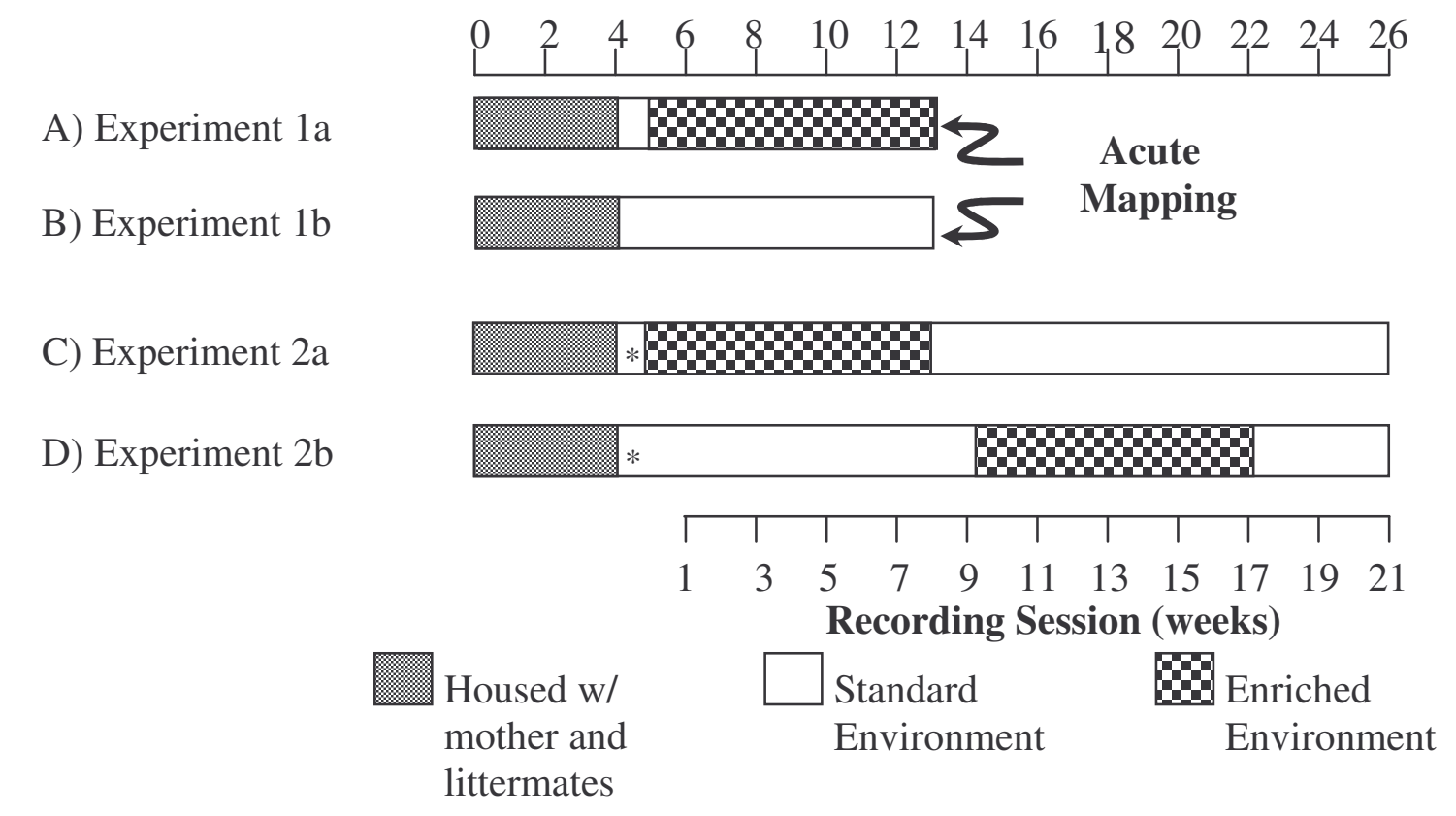

Figure 3.2. Experimental time-lines. A\&B) Experiment 1: Extracellular recordings were compared from rats housed in the enriched environment or the standard condition for 8 weeks. C\&D) Experiment 2: Evoked potentials from auditory cortex were recorded weekly for 5 months. Asterisks indicate chronic electrode implantation. In Experiment 2a (earlyenriched), rats were housed in the enriched condition for 8 weeks (from 5-13 weeks of age), and then switched to the standard condition and housed singly until 26 weeks of age. In Experiment 2b (late-enriched), rats were housed in the standard condition for 9 weeks, then switched to the enriched condition for 8 weeks (from 14-22 weeks of age), and finally, back to the standard condition until 26 weeks of age. Although enriched rats experienced two novel housing conditions, we observed no evidence of distress in any of the rats at any time. Rats actively explored the environment, voluntarily activated sound sources, and engaged in playful behavior. A male rat was introduced to the enriched environment after 4 weeks of housing to encourage natural, social interactions. 
Stimulus Presentation and Data Analysis

Sounds were delivered in a shielded double-walled sound-attenuated chamber via a speaker (Motorola model \# 40-1221) positioned directly opposite the contralateral (left) ear at a distance of $10 \mathrm{~cm}$. Frequency and intensity calibrations were performed with an ACO Pacific microphone (PS9200-7016) and Tucker-Davis SigCal software. After collecting frequency-intensity tuning curves at each site, Brainware (Tucker-Davis Technologies) presented tones at 12 repetitions of 14 modulation rates $(3-20 \mathrm{~Hz})$ and noise bursts at 4 repetition rates $(5,10,15$ and $20 \mathrm{~Hz})$. The tone frequency was selected to generate the strongest response at each recording site. A 2 second silent period separated each randomly interleaved train. All stimuli were presented at $70 \mathrm{~dB}$ SPL and were $25 \mathrm{~ms}$ long with $3 \mathrm{~ms}$ rise and fall times.

Temporal processing was quantified with 3 different measures. Tone and noise burst repetition rate transfer functions (RRTF) were derived at each site by quantifying action potentials per stimulus. Action potentials per stimulus was simply the average response occurring between 8 and $38 \mathrm{~ms}$ after the $2^{\text {nd }}, 3^{\text {rd }}, 4^{\text {th }}, 5^{\text {th }}$, and $6^{\text {th }}$ sounds in each train minus the spontaneous rate. The responses to tone and noise burst trains were also quantified using vector strength and Rayleigh statistic measures (Liang and Wang, 2002). Vector strength (VS) quantifies the degree of synchronization between action potentials and repeated tones pips, and is calculated with the formula: 


$$
\mathrm{VS}=\frac{1}{n} \sqrt{x^{2}+y^{2}} x=\sum_{i=1}^{n} \cos \theta_{i} y=\sum_{i=1}^{n} \sin \theta_{i} \theta_{i}=2 \pi \frac{t_{i}}{T}
$$

where $n=$ total number of action potentials, $t_{i}$ is the time of occurrence of the $i$ 'th action potential, and $\mathrm{T}$ is the interstimulus interval. A value of 1 indicates perfect synchronization and 0 indicates no synchronization. Rayleigh statistic $\left(2 \mathrm{nVS}^{2}\right.$, where $\mathrm{n}$ is the total number of action potentials) is a circular statistic that essentially combines the previous two measures (action potentials and vector strength) to assess the statistical significance of the vector strength (Mardia and Jupp, 2000). Values greater than $13.8(\mathrm{P}<0.001)$ are considered significant (Liang and Wang, 2002). The number of evoked action potentials, vector strength, and Rayleigh statistic at each repetition rate and the best rate for each measure were compared across groups using unpaired 2-tailed t-tests.

\section{Experiment 2 - Evoked Potential Recordings}

\section{Experimental Time-course}

Evoked potentials were recorded each week from an electrode implanted over left auditory cortex. Twenty-one female Sprague-Dawley rats were randomly assigned to 1 of 2 groups. Rats in experiment $2 \mathrm{a}(\mathrm{n}=12)$ were housed in the enriched environment for 8 weeks (5-13 wk of age), and then moved to the standard condition and housed singly until 26 weeks of age (Figure $3.2 \mathrm{C})$. Rats in experiment $2 \mathrm{~b}(\mathrm{n}=9)$ were housed singly in the standard condition for 9 weeks, then moved to the enriched environment for 8 weeks (14-22 wk of 
age), and then back to the standard condition until 26 weeks of age (Figure 3.2 D). In this series of experiments, 4-8 rats were housed in the enriched environment at any given time.

\section{Chronic Implantation}

Rats were chronically implanted at 28 days of age with a ball electrode over A1 and a ground screw over the cerebellum. Surgical anesthesia was induced with sodium pentobarbital $(50 \mathrm{mg} / \mathrm{kg}$, i.p.). A state of areflexia was maintained throughout the surgery, and supplemental doses of dilute pentobarbital were administered subcutaneously if needed $(0.2 \mathrm{ml} ; 8 \mathrm{mg} / \mathrm{ml})$. Anesthesia level was monitored by response to toe pinch. Atropine (1 $\mathrm{mg} / \mathrm{kg}$ ) and dexamethasone (4 $\mathrm{mg} / \mathrm{kg}$ ) were administered subcutaneously to minimize secretions and brain edema during the procedure and recovery. Animals received antibiotic injections (ceftriaxone $20 \mathrm{mg} / \mathrm{kg}$ ) before and after surgery. Body temperature was maintained at $37^{\circ} \mathrm{C}$. Four to five structural screws were used to anchor the implant on the skull. The 4-pin connector was held in place with dental acrylic.

Although some implants remained firmly in place for 5 months, many implants were lost due to skull growth following implantation. Implanted rats were housed singly when not in the enriched environment to minimize the tendency of rats in small cages to damage implants by grooming excessively.

\section{Stimulus Presentation and Data Analysis}

Middle latency evoked potential data was collected once each week for 21 weeks from each rat in a sound-attenuated booth. Recordings were made during the dark cycle in both housing conditions to encourage rats to be as alert as possible. However, the rats did 
spend some time sleeping. EEG recordings indicate that rats were in slow wave sleep no more than $25 \%$ of the time during each recording session. Overall, no differences in activity level, exploration, time spent sleeping, or arousal levels were noted between enriched and standard rats during recording sessions.

Acoustic stimuli included pairs of $25 \mathrm{~ms} 9-\mathrm{kHz}$ tones with interstimulus intervals of either $500 \mathrm{~ms}, 200 \mathrm{~ms}, 100 \mathrm{~ms}$, or $50 \mathrm{~ms}$. Tones pairs were presented from a speaker centered above the cage and randomly interleaved every $10 \mathrm{~s}$. The stimuli used for the awake recordings correspond to the $2,5,10$, and $20 \mathrm{~Hz}$ intervals used in experiment 1 . All stimuli were $70 \mathrm{~dB}$ SPL with $3 \mathrm{~ms}$ rise and fall times. Signals were low-passed filtered (800 $\mathrm{Hz}$ ), amplified (10,000X), and displayed on an oscilloscope for monitoring. Data acquisition computers collected cortical responses to 125 tone pair presentations. Trials with excessive motion artifacts $(>0.1 \mathrm{mV})$ were discarded prior to analysis of the mean evoked potential.

The first negative peak in the evoked response is referred to as N1 ( 40 ms); (Figure 3.6 A). The $2^{\text {nd }}$ negative peak is $\mathrm{N} 2(\sim 140 \mathrm{~ms})$. The first positive peak is referred to as P1 ( $75 \mathrm{~ms}$ ). Only the 8 rats (of the 21 implanted) that maintained their implants for the duration of the five-month study were included in this analysis. For each rat the response to the $2^{\text {nd }}$ tone was calculated by subtracting the response to a single tone (derived from the first pulse of the $500 \mathrm{~ms}$ tone pair) from the overlapping response to two tones separated by shorter $(200,100$, or $50 \mathrm{~ms})$ interstimulus intervals. Since we did not present the tone in isolation, we estimated the waveform of the response $500 \mathrm{~ms}$ after a single tone onset by using the waveform $500 \mathrm{~ms}$ after onset of the $50 \mathrm{~ms}$ pair of tones. The waveform was 
indistinguishable from baseline $500 \mathrm{~ms}$ after onset of the $50 \mathrm{~ms}$ pair (Figure 3.7 D). PPD was quantified as the root-mean-square of the evoked potential $10-175 \mathrm{~ms}$ after onset of the $2^{\text {nd }}$ tone (N1-P1 complex) divided by the RMS of the $1^{\text {st }}$ tone Paired t-tests were used to determine whether differences in evoked potential response strength and paired-pulse depression were statistically significant (alpha=0.01). 


\section{RESULTS}

Neurophysiologic responses were recorded from rats housed either in standard laboratory conditions or in an enriched environment. Action potentials were recorded from small groups of A1 neurons at more than 700 sites in 14 rats. Twenty-one rats were implanted with EEG electrodes and evoked responses were recorded from auditory cortex each week for up to 5 months. Although similar environmental plasticity was observed in all the rats, only the eight rats that maintained their implants for the duration of the 21-week study were included in the analysis reported below.

\section{Experiment 1 - Extracellular Recordings}

\section{Repetition Rate Transfer Functions}

Responses from animals housed in enriched ( $\mathrm{n}=8$ rats; 462 sites) and standard ( $\mathrm{n}=6$ rats; 263 sites) conditions indicate that environment substantially altered A1 responses to modulated noise bursts and tones that had no special significance in the enriched environment.

A representative example of a dot raster plot from a single site is shown in Figure 3.3 A \& B. Each point represents an action potential. Panel A of this figure shows responses to tone trains, and panel B shows responses to noise burst trains. The number of action potentials evoked per sound declines rapidly at repetition rates above $12 \mathrm{~Hz}$ (Figure $3.3 \mathrm{C}$ ). 

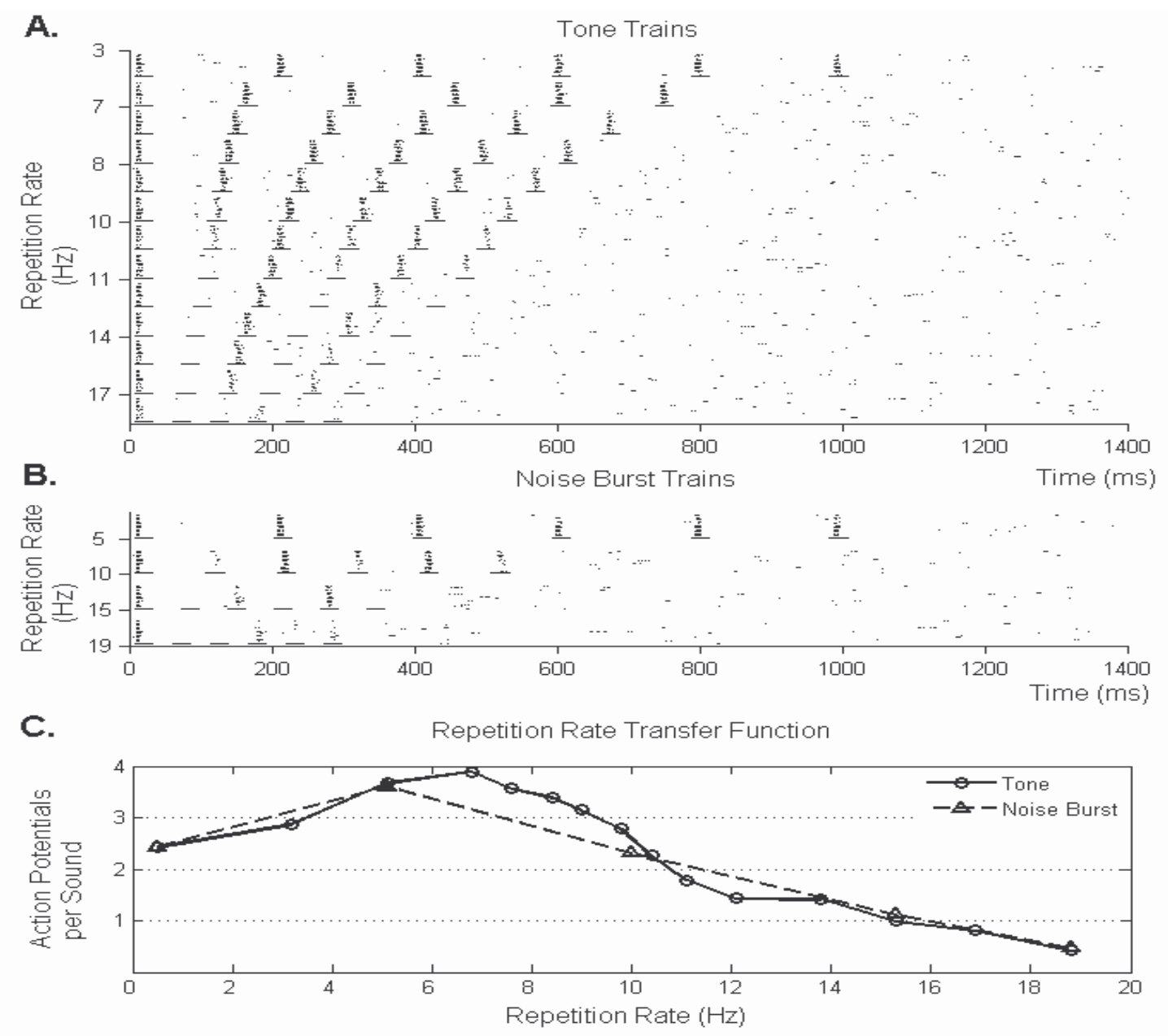

Figure 3.3. Responses of a single site in an enriched rat to repeated tone and noise burst trains. A\&B) In the spike dot-raster plots, action potential times are indicated for each of the 12 repetitions of each train. Tones were presented at 14 different repetition rates $(3-19 \mathrm{~Hz})$ and noise burst trains were presented at 4 rates $(5-19 \mathrm{~Hz})$. The tone frequency of $19-\mathrm{kHz}$ was selected because it was close to the site's best frequency of $23 \mathrm{kHz}$. C) Repetition rate transfer functions (RRTF) for tones (straight line) and noise burst trains (dashed line) for the same sites shown in A and B. 
The mean RRTF from enriched and standard rats is shown in Figure 3.4 A. At slower rates $(<8 \mathrm{~Hz})$, A1 neurons from enriched rats responded with significantly more action potentials per tone compared to naïve rats. At faster rates however, A1 neurons from enriched rats responded with significantly fewer action potentials per tone. The rate that evoked the maximum number of action potentials per tone at each site (best modulation rate) was significantly decreased in enriched rats compared to standard rats $(6.3 \pm 0.2 \mathrm{~Hz}$ vs. $7.5 \pm$ $0.3 \mathrm{~Hz}, \mathrm{p}<0.001$ ). The limiting rate (defined as the highest rate at which the response was $50 \%$ of the response to the best modulation rate) was also significantly lower in enriched rats $(10.4 \pm 0.2 \mathrm{~Hz}$ vs. $12.3 \pm 0.2 \mathrm{~Hz}, \mathrm{p}<0.000001)$

The degree of synchronization between tone trains and action potentials was quantified with vector strength and the Rayleigh statistic (see Methods). Enrichment significantly decreases the degree of phase locking at high repetition rates and increases it at low rates (Figure 3.4 B). The rate at which maximum phase locking occurred was lower in enriched rats compared to standard rats $(8.92 \pm 0.14$ vs. $10.66 \pm 0.23, \mathrm{p}<0.000001)$. The average rate that resulted in the highest Rayleigh statistic for each site was also lower $(7.6 \pm$ 0.1 and $8.9 \pm 0.2, \mathrm{p}<0.000001)$. Both groups showed significant phase locking at all rates (Figure $3.4 \mathrm{C}$ ). The average vector strength at the best modulation rate for each site was not significantly different between the two groups $(0.88 \pm 0.01$ vs. $0.88 \pm 0.01, \mathrm{p}>0.05)$. The observation that enriched rats exhibited increased response strength and synchronization at slow rates, but decreased response strength and synchronization at fast rates indicates that enrichment decreased the preferred modulation rates of A1 neurons. 
A.

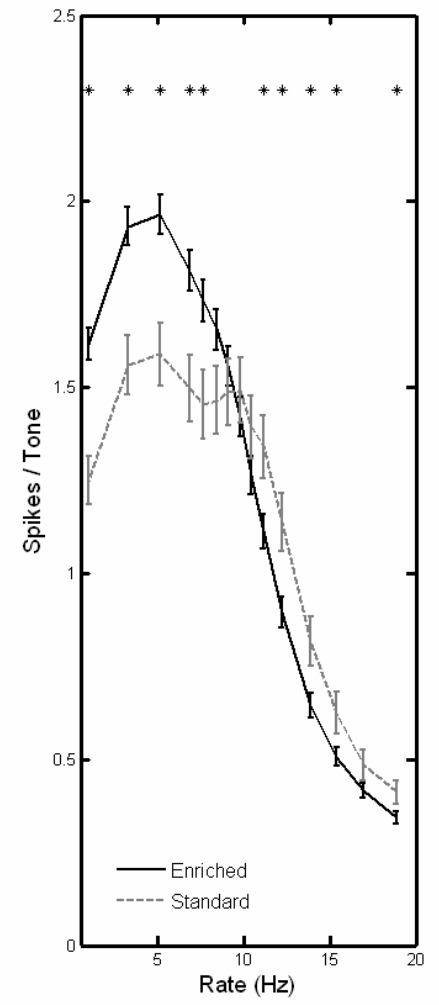

B.

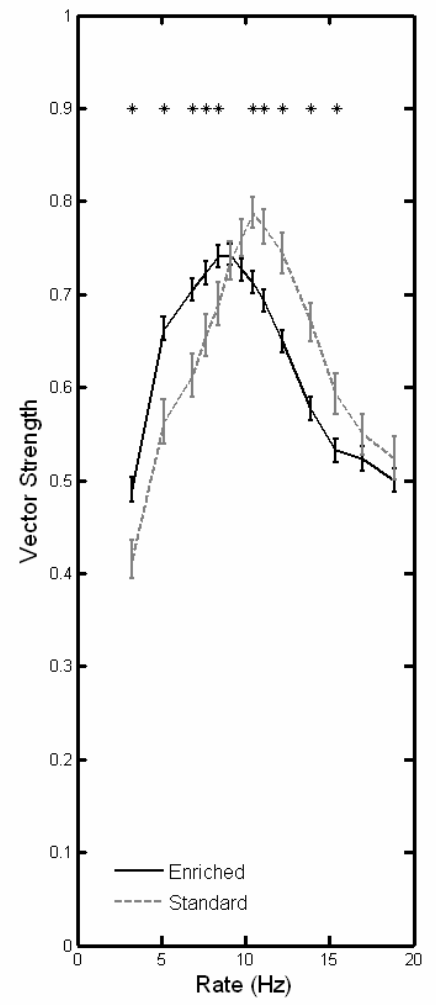

C.

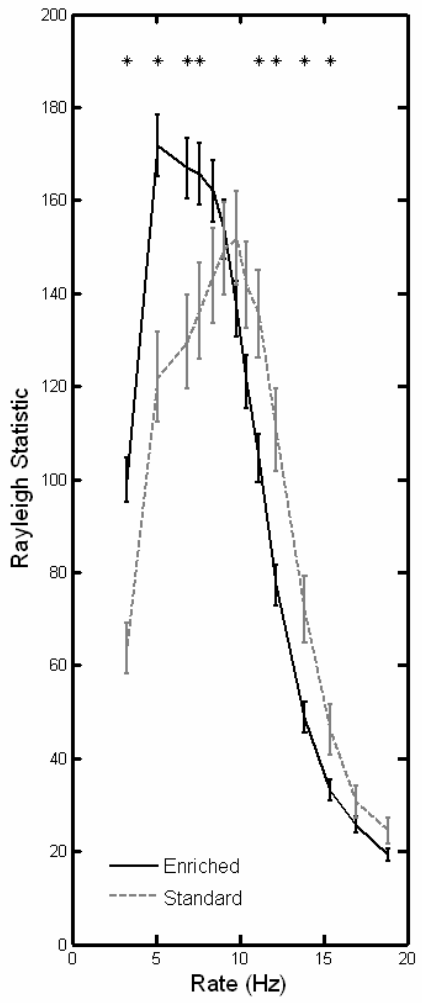

Figure 3.4. Repetition rate transfer function, vector strength, and Rayleigh statistic for tone trains. A) Average repetition rate transfer functions for tones in enriched (solid black) and standard (dashed grey) rats. The tone frequency was set to the best frequency for each site. B) Vector strength quantifies the degree of synchronization between action potentials and repeated tone onset. C) The Rayleigh statistic combines the degree of synchronization with the number of action potentials. These results indicate that compared to standard-housed controls, enriched rats exhibited increased response strength and synchronization at slow rates, but exhibited decreased response strength and synchronization at fast rates. Significant differences between the groups are indicated with an asterisk $(\mathrm{p}<0.05$ by two-tailed, unpaired t-test). Error bars indicate standard error of the mean. 
A1 neurons from enriched rats also responded with more action potentials to noise bursts when presented at rates below $10 \mathrm{~Hz}$ (Figure 3.5). Once again, the average vector strength at the best modulation rate for each site was not significantly different between the two groups ( $0.86 \pm 0.01$ vs. $0.87 \pm 0.01, \mathrm{p}>0.05)$.

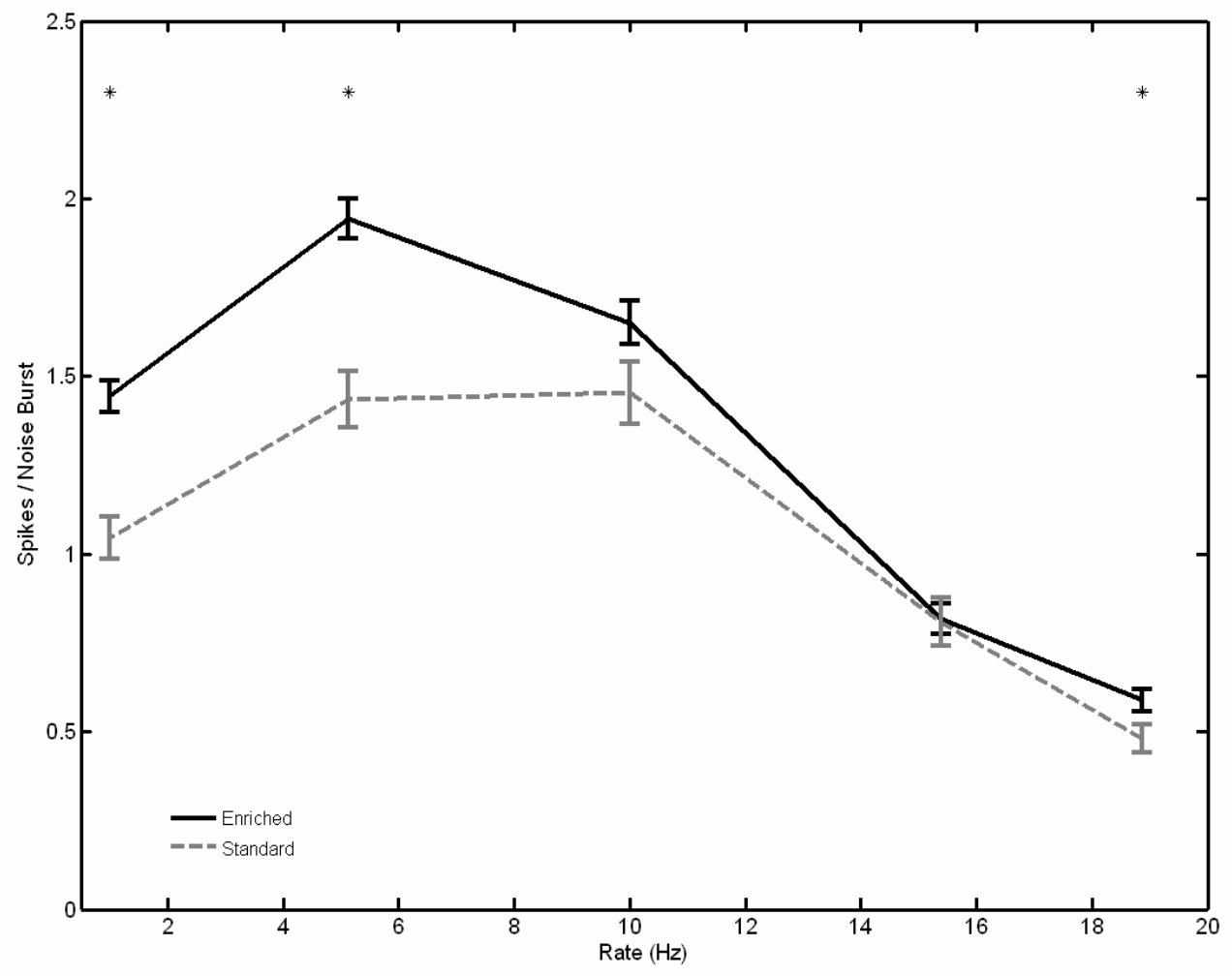

Figure 3.5. Repetition rate transfer function for noise bursts. Average repetition rate transfer functions for noise burst trains in enriched (solid black) and standard (dashed grey) rats. At slow rates $(<10 \mathrm{~Hz})$ the number of action potentials evoked per noise burst was greater for enriched rats compared to standard-housed rats. Significant differences between the groups are indicated with an asterisk ( $\mathrm{p}<0.05$ by two-tailed, unpaired t-test). Error bars indicate standard error of the mean. 


\section{Experiment 2 - Evoked Potential Recordings}

\section{Tone-evoked Responses}

To determine if enrichment also increases response strength and paired-pulse depression in unanaesthetized rats and whether or not this increase is reversible, we recorded auditory evoked potentials weekly during periods of standard and enriched housing. We previously reported that response strength increased significantly during enrichment and reversed within a week when rats were returned to standard housing conditions. Chronic recordings from awake rats indicate that paired-pulse depression is also increased during periods of enrichment and returns to normal levels during standard housing (Figure 3.6 A, B).

To improve the signal to noise ratio, we compared the average of each individual's mean evoked potential for all presentations during the standard housing condition, including before and after enrichment, with all presentations during the enriched housing condition. The amplitudes of the N1, P1, and N2 peaks in the grand mean average response to the first tone during enrichment were $211 \%, 243 \%$, and $167 \%$ of their amplitude during standard housing (Figure $3.7 \mathrm{~A}$ ). The amplitudes of each peak in response to a $2^{\text {nd }}$ tone presented 500 ms later were $163 \%, 182 \%$, and $167 \%$ of their amplitude during standard housing (Figure 3.7 A). These results suggest that enrichment causes greater paired-pulse depression by increasing the $\mathrm{N} 1$ and $\mathrm{P} 1$ responses of the $1^{\text {st }}$ tone compared to the $2^{\text {nd }}$ tone. 

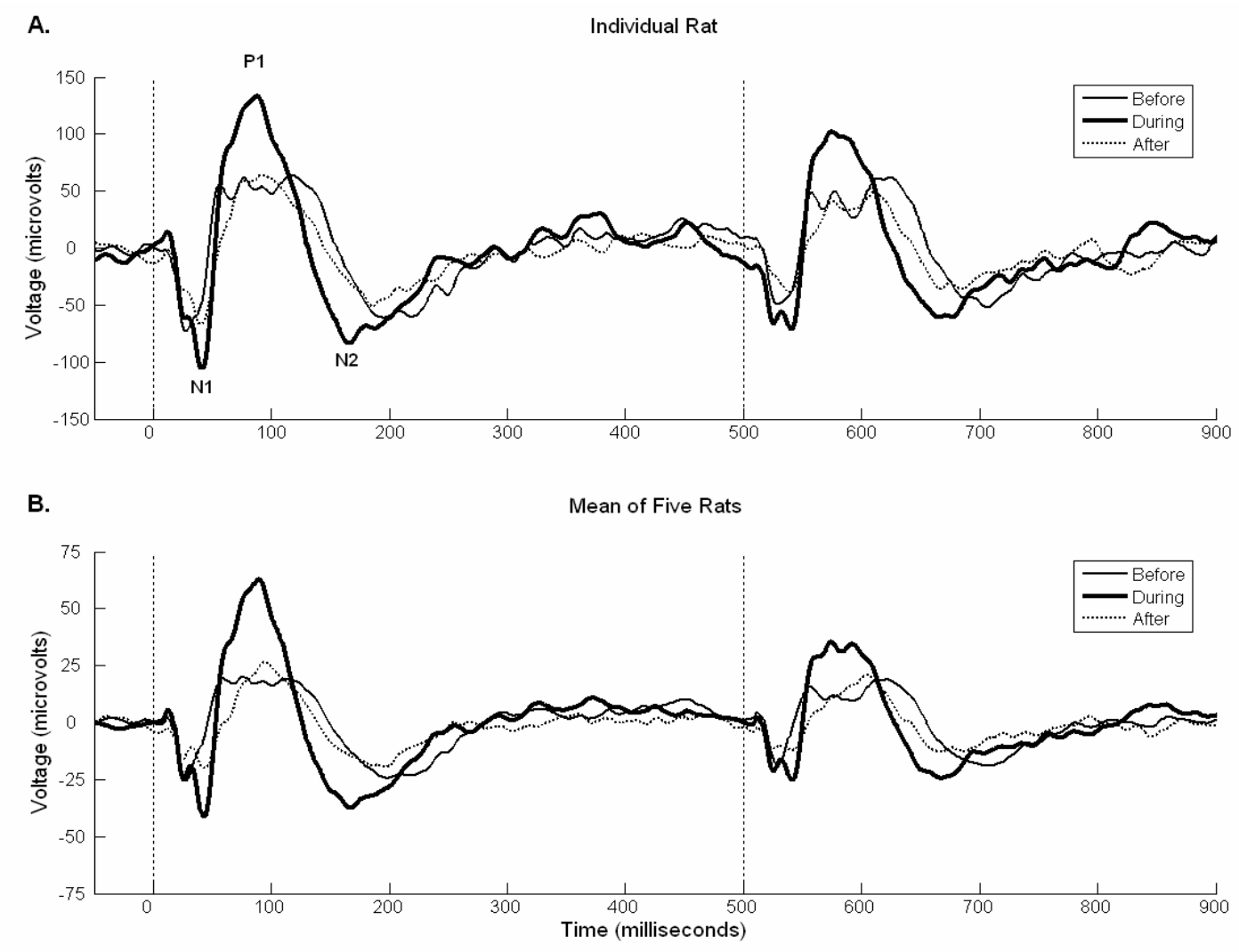

Figure 3.6. Average tone-evoked response before, during, and after enrichment. A) Mean auditory evoked potentials from an individual rat before, during, and after enrichment in response to two $70 \mathrm{~dB} 9-\mathrm{kHz}$ tones separated by $500 \mathrm{~ms}$. B) Grand mean average auditory evoked potentials from 5 rats before, during, and after enrichment (Experiment 2b). These results indicate that response amplitude and paired-pulse depression are increased during periods of enrichment and return to normal levels during standard housing. 


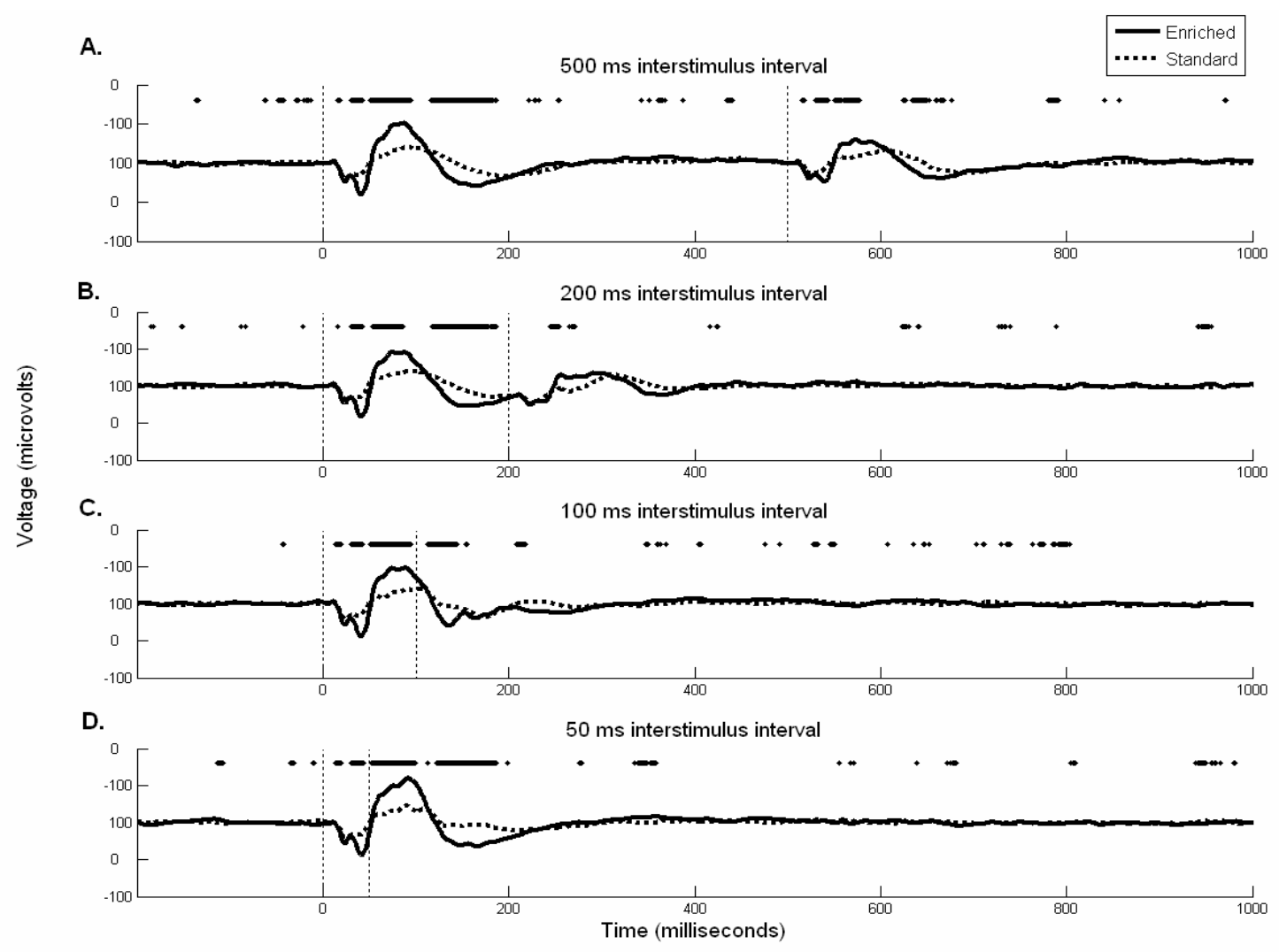

Figure 3.7. Average tone-evoked response for enriched compared to standard-housed rats. Grand mean average responses of enriched (solid) and standard (dashed) rats to two $9-\mathrm{kHz}$ tones separated by 500 (A), 200 (B), 100 (C), and 50 (D) ms intervals. The milliseconds during which the enriched and standard potentials are significantly different from each other are indicated with dots (paired two-tailed t-tests $\mathrm{p}<0.01$ ). These results indicate that pairedpulse depression increases more rapidly as interstimulus interval decreases during enriched compared to standard housing. 
The surface potential in response to the $2^{\text {nd }}$ of 2 tones separated by $500 \mathrm{~ms}$ was smaller than the $1^{\text {st }}$ when rats were housed in the standard and the enriched conditions. The peak-to-peak amplitude of the response to the $2^{\text {nd }}$ tone was reduced by $11 \%$ during standard housing and by 37\% during enrichment (Figure 3.7 A). Since the evoked responses of some rats were different from the grand mean average, we used the root-mean-square of the N1-P1 complex to quantify the power of the average evoked potential in each individual. Paired-pulse depression was quantified as the ratio of the response to the $2^{\text {nd }}$ tone divided by the response to the $1^{\text {st }}$. For the $500 \mathrm{~ms}$ interstimulus interval, this ratio was $64 \pm 4 \%$ for rats during periods of housing in the enriched environment compared to $87 \pm 7 \%$ for rats during periods of housing in the standard condition ( $\mathrm{p}<0.05$; Figure 3.9). Collectively, these results indicate that enrichment significantly increases the response to the $1^{\text {st }}$ tone and significantly enhances the degree of paired-pulse depression of the $2^{\text {nd }}$ tone $500 \mathrm{~ms}$ later compared to standard housing.

During enrichment, paired-pulse depression was even greater at interstimulus intervals shorter than $500 \mathrm{~ms}$. Since the response to a single tone lasts $250 \mathrm{~ms}$, the response to the $2^{\text {nd }}$ tone overlapped at shorter interstimulus intervals (Figure 3.7 B-D). To quantify the response to the $2^{\text {nd }}$ tone, we subtracted the waveform from the response to the $1^{\text {st }}$ tone alone. This analysis assumes that the 2 responses summate linearly. The response to the $2^{\text {nd }}$ tone decreased more rapidly as the interstimulus interval decreased in enriched compared to standard-housed rats. During enrichment, the grand mean average peak-to-peak amplitude evoked by the $2^{\text {nd }}$ of two tones separated by 200,100 or 50 ms was reduced by $60 \%, 70 \%$, or $80 \%$, respectively, compared to the response to the $1^{\text {st }}$ tone (Figure $3.8 \mathrm{~A}$ ). In contrast, during 
standard housing the response to the $2^{\text {nd }}$ tone was reduced by $35 \%, 24 \%$, or $41 \%$ (Figure 3.8 B). The root-mean-square power of the evoked response of individual rats also showed the same pattern. Significant paired-pulse depression was observed at each of these intervals regardless of housing condition $(\mathrm{p}<0.05)$; however, there was significantly more paired-pulse depression when rats were housed in an enriched environment compared to standard housing $\left(\mathrm{p}<0.01\right.$; Figure 3.9). During enriched housing, the power of the response to the $2^{\text {nd }}$ tone was reduced by $50 \%$ when the interval was decreased from 500 to 50 ms. During standard housing, the response only decreased by $20 \%$. While paired-pulse depression increased as the interstimulus interval decreased when rats were housed in the enriched environment $(\mathrm{p}<0.01)$, there was no significant difference in the degree of paired-pulse depression during standard housing. These results indicate that enrichment substantially increases the degree of response suppression caused by preceding sounds. 

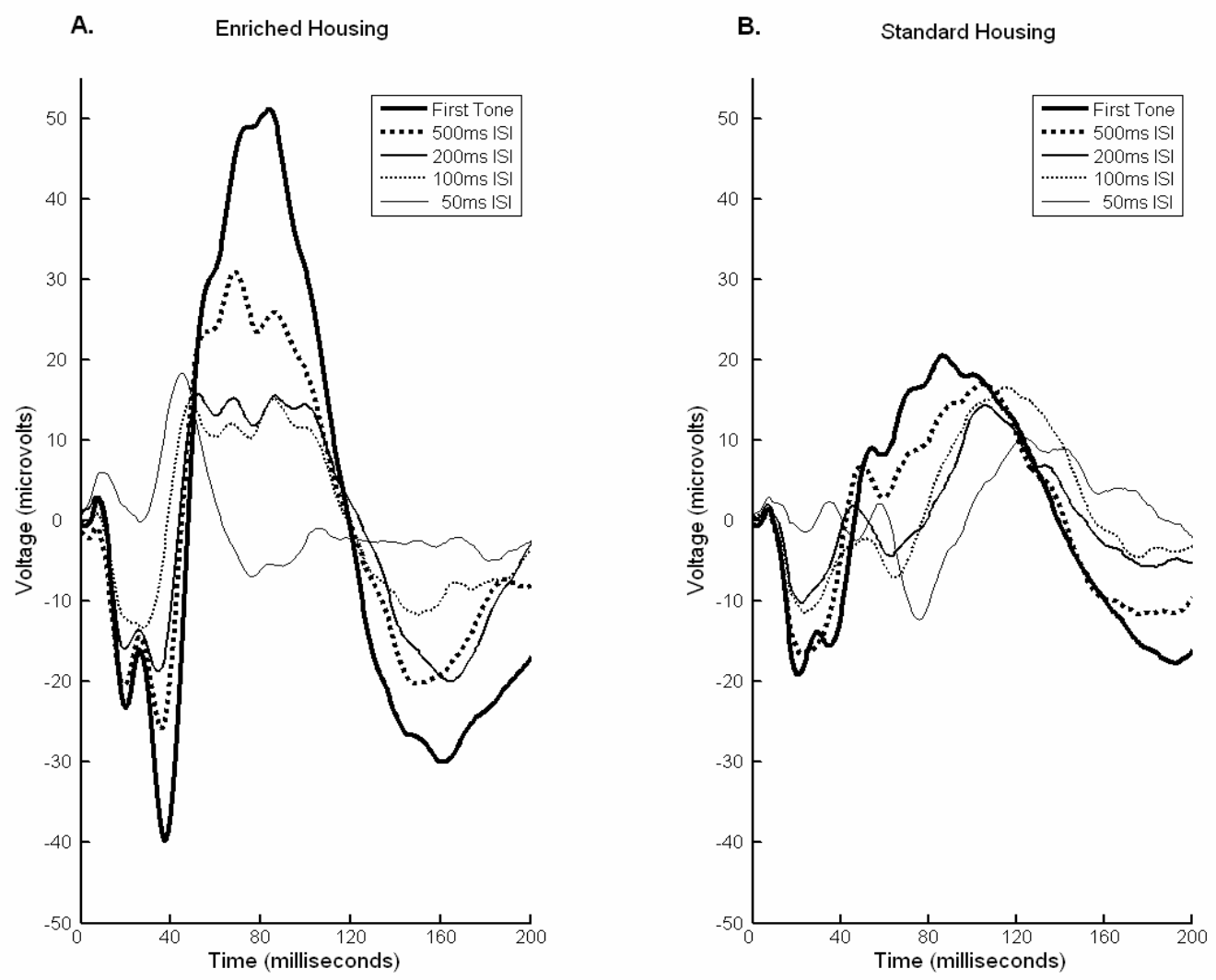

Figure 3.8. Overlays of the mean response evoked by each tone when presented in isolation or 500 to $50 \mathrm{~ms}$ after another tone. For the 200, 100, and $50 \mathrm{~ms}$ intervals, the responses of each rat were subtracted from their response to a single tone (derived from the first pulse of the $500 \mathrm{~ms}$ tone pair). Paired-pulse depression increases more rapidly as the interval is decreased during enriched compared to standard housing. 


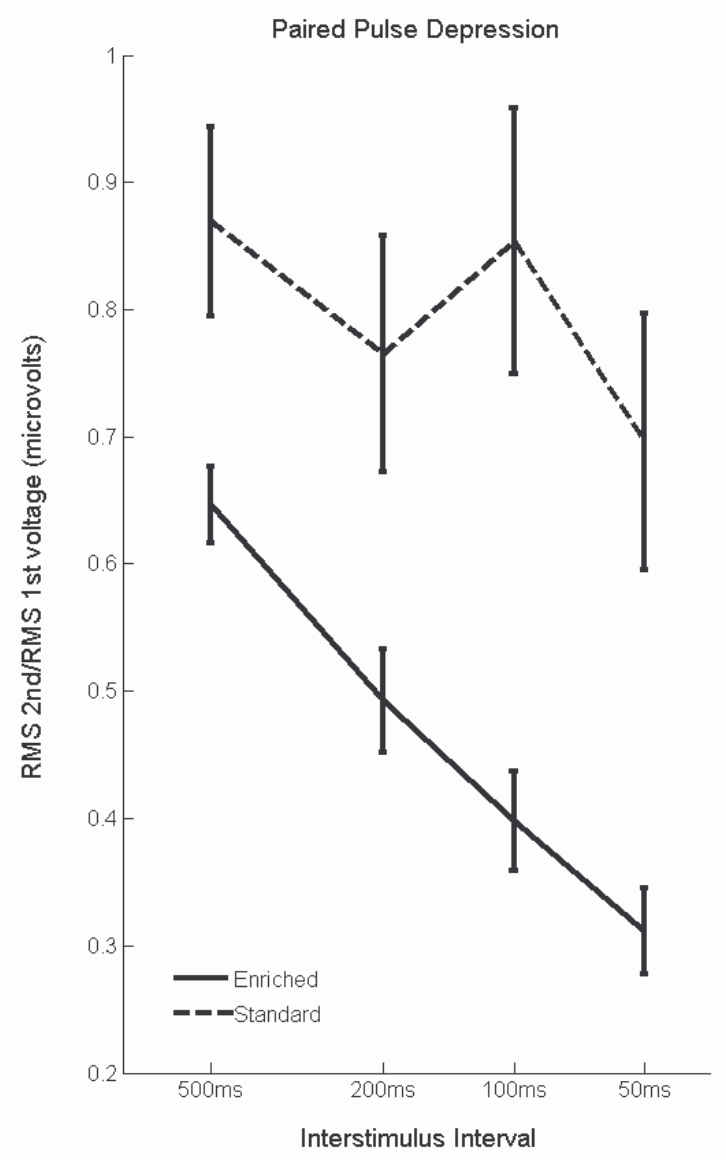

Figure 3.9. Average paired-pulse depression ratios for each group as a function of interstimulus interval. The paired-pulse depression ratio was quantified as root-mean-square of the response to the $2^{\text {nd }}$ tone (10-175 ms after tone onset) divided by the root-mean-square of the response to the $1^{\text {st }}$. Error bars indicate standard error of the mean. Evoked potentials exhibited significant paired-pulse depression in both groups at all rates tested. Paired-pulse depression was greater during enrichment, compared to standard housing, at all rates tested. Paired-pulse depression was significantly greater at $50 \mathrm{~ms}$ compared to $500 \mathrm{~ms}$ during enrichment but not during standard housing. 


\section{DISCUSSION}

Our previous study demonstrated that environmental enrichment substantially increases the response of rat auditory cortex neurons to tone and noise bursts stimuli (Engineer et al., 2004). The current study was designed to evaluate the neurophysiologic consequences of environmental enrichment on temporal information processing in the auditory cortex. Action potentials from anesthetized rats were collected in response to tone and noise burst trains of varying repetition rates. Compared to standard-housed rats, A1 responses from enriched rats were stronger and more synchronized at rates below $10 \mathrm{~Hz}$, and weaker and less synchronized at higher rates. Evoked potentials were also recorded from auditory cortex in awake rats to compare the degree of paired-pulse depression during housing in standard and enriched conditions. Paired-pulse depression increased while rats were housed in the enriched environment and returned to normal levels within days of transfer back to standard housing. Collectively, these studies demonstrate that environment can substantially alter temporal processing in the auditory cortex of anesthetized and awake rats.

\section{Temporal Processing}

Neurons in the visual, auditory, and somatosensory cortex fail to respond at high modulation rates. Repetition rate transfer functions in the primary auditory cortex of naïve monkeys, cats, and rats are predominantly low-pass or band-pass (Gaese and Ostwald, 1995; Kilgard and Merzenich, 1998; Eggermont, 1999; Beitel et al., 2003; Bao et al., 2004). Neurons with shorter latencies typically exhibit faster recovery from forward masking and 
higher best repetition rates than neurons with longer latencies (Brosch and Schreiner, 1997; Schreiner et al., 1997; Kilgard and Merzenich, 1999). Our observation that enrichment increases time to peak and decreases best repetition rate of A1 neurons is consistent with this correlation. The enrichment induced decrease in the maximum following rate and increased paired-pulse depression is not due to a greater number of sounds with slow modulation rates in the enriched environment compared to the standard environment. In fact, the average modulation rate in the enriched environment was higher due to the greater complexity of the sound sources (music, chimes, etc).

One potential consequence of enrichment may be more accurate perceptual judgments to sounds with modulation rates below $10 \mathrm{~Hz}$, given the greater response strength and synchronization to these sounds. However, the greater paired-pulse depression observed in enriched rats may impair their ability to detect stimuli in a forward masking protocol. Detailed psychophysical experiments will be needed to fully evaluate the perceptual consequences of environmental enrichment. In any case, enriched housing provides sensory stimulation and social interactions that are common in natural environments and it is likely that the greater paired-pulse depression observed in enriched rats more accurately reflects typical cortical temporal information processing compared to results from animals housed in standard laboratory conditions.

\section{Potential Mechanisms}

Plasticity of synaptic, cellular, or network properties may contribute to the changes in temporal processing induced by environmental conditions (Katz and Shatz, 1996; Gilbert, 
1998; Syka, 2002). Two distinct types of excitatory synapses have been described in layer II/III auditory cortex neurons of standard-housed rats (Atzori et al., 2001). Two-thirds have weak connection strengths with a low probability of release, while the remaining synapses have strong connection strengths and a high probability of release. The strong synapses exhibited substantially more paired-pulse depression than the weak synapses, presumably due to faster depletion of the synaptic vesicle pool. Earlier studies have shown that longterm potentiation of cortical synapses is often accompanied by higher probability of release and increased paired-pulse depression (Markram and Tsodyks, 1996). If enrichment increases the proportion of synapses with a high release probability, both our earlier observation of increased response strength and our current observation of increased response depression could be explained by a single mechanism.

Enrichment could also strengthen paired-pulse depression by increasing long-lasting inhibition. However, in cat visual cortex, enrichment decreases rather than increases the number of inhibitory synapses, and increases rather than decreases the ability of neurons to follow rapid stimuli (Beaulieu and Colonnier, 1987; Beaulieu and Cynader, 1990). Although enrichment resulted in increased responsiveness and selectivity similar to that seen in rat auditory cortex, the opposite effects on temporal processing suggest that different synaptic mechanisms may be involved.

Recent in vivo findings indicate that at interstimulus intervals over 150 ms synaptic depression, rather than inhibition, is the primary mechanism responsible for paired-pulse depression in rat auditory cortex (Wehr and Zador, 2003, 2005; Tan et al., 2004). While it is possible that enrichment could strengthen paired-pulse depression by increasing long-lasting 
inhibition (or even the amplitude of post-response hyperpolarization or oscillatory conductance periodicity), each of these explanations would also require an increase in excitatory drive to explain the stronger response to isolated sounds (Gaese and Ostwald, 1995; D'Angelo and Naidi, 2001; He, 2003).

The increased paired-pulse depression observed in enriched rats is likely to be a consequence of the increased response magnitude to the first tone in a pair. Several experiments have shown that greater stimulus intensity causes greater paired-pulse depression. Increasing tone amplitude typically increased the duration of forward masking (Brosch and Schreiner, 1997). In addition, we observed more paired-pulse depression of evoked potentials with louder $(90 \mathrm{~dB})$ tones compared to quieter $(50 \mathrm{~dB})$ tones in standardhoused rats (unpublished data). These results suggest enrichment has the same effect on response strength and paired-pulse depression as increasing stimulus intensity.

Although numerous cellular mechanisms are possible, increased probability of synaptic release is the most likely explanation since a single mechanism (well documented with in vitro studies of auditory cortex) would explain both the increased response strength and decreased temporal following rate observed in enriched rat auditory cortex. Clearly, further studies are needed to characterize the cellular and molecular basis of enrichmentinduced plasticity.

Finally, since both response strength and paired-pulse depression increase during the transition from an alert state to a quiet awake state, it is possible that enriched rats spend more time in the quiet awake state than standard-housed rats (Castro-Alamancos, 2004). However, since the enrichment-induced increases in response strength and paired-pulse 
depression are maintained under general anesthesia, we believe that differences in global state are unlikely to explain our observations.

\section{Technical Considerations}

Both standard and enriched rats have significant paired-pulse depression to subsequent auditory stimuli. However, the degree of paired-pulse depression was different depending on the method of data recording. For surface potentials recorded in awake rats, the time required for the amplitude of the $2^{\text {nd }}$ response to recover to two-thirds of the $1^{\text {st }}$ response was ten times longer in enriched compared to standard-housed rats (500 vs. $50 \mathrm{~ms})$. In contrast, for action potentials recorded under anesthesia, the time required for the response to the $2^{\text {nd }}$ tone to recover to two-thirds of the $1^{\text {st }}$ was only $17 \%$ longer $(83 \mathrm{vs} .71 \mathrm{~ms})$. Three factors could be responsible for the longer time-course of paired-pulse depression for evoked potentials compared to action potentials. First, anesthesia may eliminate possible neuromodulatory differences that could be engaged in awake rats during periods of differential housing. Second, non-primary auditory regions, which likely contribute to evoked potentials, are not included in our microelectrode data. Non-primary auditory cortex neurons are known to exhibit greater paired-pulse depression (Eggermont and Ponton, 2002) and may be more sensitive to environmental conditions. Third, evoked potentials are generated by summed synaptic potentials, which appear to be potentiated during enrichment. Recordings of action potentials would show smaller differences due to the threshold-based mechanism of action potential generation. Further studies will be needed to determine which 
of these differences is responsible for the greater scale of environmental plasticity observed with evoked potentials.

Multiple factors influence the degree of environmental-induced plasticity including physical activity, social experience, and behavioral relevance of sensory events (van Praag et al., 2000). Social interactions significantly increase brain weight (Ferchmin and Bennett, 1975) and wheel running increases cell proliferation and neurogenesis in the adult mouse dentate gyrus (van Praag and Gage, 1999). Additional experiments are ongoing to evaluate which specific factors contribute to the physiologic plasticity observed in this study.

\section{Clinical Relevance}

Several clinical populations exhibit abnormal cortical responses to rapidly presented acoustic signals. Unmedicated patients with schizophrenia exhibit weak responses and less paired-pulse depression compared to normal controls, possibly reflecting their inability to filter incoming sensory stimuli and reported sensations of being "overwhelmed" or "flooded" by sensory stimulation (Siegel et al., 1984; Braff and Geyer, 1990; Erwin et al., 1991). Paired-pulse depression is restored to normal when patients are treated with the antipsychotic clozapine (Adler et al., 2004). Individuals with autism also exhibit reduced responses to sensory stimulation that fail to adapt at increased repetition rates (Buchwald et al., 1992). These differences may contribute to abnormal gating of sensory information, abnormal arousal levels, and inattentiveness characteristic of the autistic population.

In contrast, individuals with dyslexia exhibit too much paired-pulse depression. The response to the first acoustic stimulus is stronger, but the response to $2^{\text {nd }}$ and subsequent 
stimuli is significantly weaker compared with controls (Nagarajan et al., 1999). These differences were especially pronounced at short interstimulus intervals, which is consistent with observations that dyslexic individuals have substantial difficulties processing the rapid spectrotemporal transitions present in language. Although the perceptual consequences of environmental enrichment are not well documented, our observation that PPD can be increased or decreased as a function of experience, supports earlier hypotheses that focused, intensive sensory training may alter sensory gating in patients with schizophrenia, autism, or dyslexia (Baranek, 2002; Gunatilake and Silva, 2004; Tallal et al., 1998).

\section{Conclusion}

In summary, enrichment strengthens the response of primary auditory cortex neurons to isolated sounds but increases the degree of paired-pulse depression. Both forms of plasticity could be explained if enrichment increases the probability of synaptic release, although several other mechanisms are possible, and may act in concert. A better understanding of the cellular basis for enrichment-induced paired-pulse depression would be helpful in designing effective treatments for neurological disorders characterized by temporal processing abnormalities. 


\section{REFERENCES}

Adler, L.E., Olincy, A., Cawthra, E.M., McRae, K.A., Harris, J.G., Nagamoto, H.T., Waldo, M.C., Hall, M.H., Bowles, A., Woodward, L., Ross, R.G. and Freedman, R. (2004) Varied effects of atypical neuroleptics on P50 auditory gating in schizophrenia patients. Am J Psychiatry 161: 1822-1828.

Atzori, M., Lei, S., Evans, D.I., Kanold, P.O., Phillips-Tansey, E., McIntyre, O. and McBain, C.J. (2001). Differential synaptic processing separates stationary from transient inputs to the auditory cortex. Nat Neurosci 4: 1230-1237.

Bao, S., Chang, E.F., Woods, J. and Merzenich, M.M. (2004) Temporal plasticity in the primary auditory cortex induced by operant perceptual learning. Nat Neurosci 7: 974981.

Baranek, G.T. (2002). Efficacy of sensory and motor interventions for children with autism. $J$ Autism Dev Disord 32: 397-422.

Beaulieu, C. and Colonnier, M. (1987). Effect of the richness of the environment on the cat visual cortex. J Comp Neurol 266: 478-494.

Beaulieu, C. and Cynader, M. (1990). Effect of the richness of the environment on neurons in cat visual cortex. II. Spatial and temporal frequency characteristics. Dev Brain Res 53: 82-88.

Beitel, R.E., Schreiner, C.E., Cheung, S.W., Wang, X. and Merzenich, M.M. (2003).

Reward-dependent plasticity in the primary auditory cortex of adult monkeys trained to discriminate temporally modulated signals. PNAS 100: 11070-11075.

Braff, D.L. and Geyer, M.A.. (1990). Sensorimotor gating and schizophrenia. Human and animal model studies. Arch Gen Psychiatry 47: 181-188. 
Brosch, M. and Schreiner, C.E. (1997). Time-course of forward masking tuning curves in cat primary auditory cortex. J Neurophysiol 77: 923-943.

Buchwald, J.S., Erwin, R., Van Lancker, D., Guthrie, D., Schwafel, J. and Tanguay, P. (1992). Midlatency auditory evoked responses: P1 abnormalities in adult autistic subjects. Electroencephalogr Clin Neurophysiol 84: 164-171.

Castro-Alamancos, M.A.. (2004). Absence of rapid sensory adaptation in neocortex during information processing states. Neuron 41: 455-464.

Clark, M.G., Rosen, G.D., Tallal, P. and Fitch, R.H. (2000a) Impaired processing of complex auditory stimuli in rats with induced cerebrocortical microgyria: an animal model of developmental language disabilities. J Cogn Neurosci 12: 828-839.

Clark, M.G., Rosen, G.D., Tallal, P. and Fitch, R.H. (2000b) Impaired two-tone processing at rapid rates in male rats with induced microgyria. Brain Res 871: 94-97.

D'Angelo, E., Nieus, T., Maffei, A., Armano, S., Rossi, P., Taglietti, V., Fontana, A. and Naidi, G. (2001). Theta-frequency bursting and resonance in cerebellar granule cells: experimental evidence and modeling of a slow K+-dependent mechanism. J Neurosci 21: 759-770.

Eggermont, J.J. (1999). The magnitude and phase of temporal modulation transfer functions in cat auditory cortex. J Neurosci 19: 2780-2788.

Eggermont, J.J. and Ponton, C.W. (2002). The neurophysiology of auditory perception: from single units to evoked potentials. Audiol Neurootol 7: 71-99.

Engineer, N.D., Percaccio, C.R., Pandya, P.K., Moucha, R., Rathbun, D.L. and Kilgard, M.P. (2004). Environmental enrichment improves response strength, threshold, selectivity, and latency of auditory cortex neurons. J Neurophysiol 92: 73-82. 
Erwin, R.J., Mawhinney-Hee, M., Gur, R.C. and Gur, R.E. (1991). Midlatency auditory evoked responses in schizophrenia. Biol Psychiatry 30: 430-442.

Ferchmin, P.A. and Bennett, E.L. (1975). Direct contact with enriched environment is required to alter cerebral weights in rats. J Comp Physiol Psychol 88: 360-367.

Fitch, R.H., Tallal, P., Brown, C.P., Galaburda, A.M. and Rosen, G.D. (1994). Induced microgyria and auditory temporal processing in rats: a model for language impairment? Cereb Cortex 4: 260-270.

Gaese, B.H. and Ostwald, J. (1995). Temporal coding of amplitude and frequency modulation in the rat auditory cortex. Eur J Neurosci 7: 438-450.

Gilbert, C.D. (1998). Adult cortical dynamics. Physiol Rev 78: 467-485.

Gunatilake, S., Ananth, J., Parameswaran, S., Brown, S. and Silva, W. (2004). Rehabilitation of schizophrenic patients. Curr Pharm Des 10: 2277-2288.

Harrington, I.A., Heffner, R.S. and Heffner, H.E. (2001). An investigation of sensory deficits underlying the aphasia-like behavior of macaques with auditory cortex lesions. Neuroreport 12: 1217-1221.

Hayes, E.A., Warrier, C.M., Nicol, T.G., Zecker, S.G. and Kraus, N. (2003). Neural plasticity following auditory training in children with learning problems. Clin Neurophysiol 114: 673-684.

He, J. (2003). Slow oscillation in non-lemniscal auditory thalamus. J Neurosci 23: 82818290.

Herman, A.E., Galaburda, A.M., Fitch, R.H., Carter, A.R. and Rosen, G.D. (1997). Cerebral microgyria, thalamic cell size and auditory temporal processing in male and female rats. Cereb Cortex 7: 453-464. 
Katz, L.C. and Shatz, C.J. (1996). Synaptic activity and the construction of cortical circuits. Science 274: 1133-1138.

Kilgard, M.P. and Merzenich, M.M. (1999). Distributed representation of spectral and temporal information in rat primary auditory cortex. Hear Res 134: 16-28.

Kilgard, M.P. and Merzenich, M.M. (1998). Plasticity of temporal information processing in the primary auditory cortex. Nat Neurosci 1: 727-731.

Kilgard, M.P., Pandya, P.K., Vazquez, J., Gehi, A., Schreiner, C.E. and Merzenich, M.M. (2001). Sensory input directs spatial and temporal plasticity in primary auditory cortex. J Neurophysiol 86: 326-338.

Liang, L., Lu, T. and Wang, X. (2002). Neural representations of sinusoidal amplitude and frequency modulations in the primary auditory cortex of awake primates. $J$ Neurophysiol 87: 2237-2261.

Mardia, K.V. and Jupp, P.E. Directional statistics. Chichester; New York: J. Wiley, 2000.

Markram, H. and Tsodyks, M. (1996). Redistribution of synaptic efficacy between neocortical pyramidal neurons. Nature 382: 807-810.

Merzenich, M.M, Jenkins, W.M., Johnston, P., Schreiner, C., Miller, S.L. and Tallal, P. (1996). Temporal processing deficits of language-learning impaired children ameliorated by training. Science 271: 77-81.

Merzenich, M.M., Schreiner, C., Jenkins, W. and Wang, X. (1993). Neural mechanisms underlying temporal integration, segmentation, and input sequence representation: some implications for the origin of learning disabilities. Ann N Y Acad Sci 682: 1-22.

Mummery, C.J., Ashburner, J., Scott, S.K. and Wise, R.J. (1999). Functional neuroimaging of speech perception in six normal and two aphasic subjects. J Acoust Soc Am 106: 449-457. 
Nagarajan, S.S., Blake, D.T., Wright, B.A., Byl, N. and Merzenich, M.M. (1998). Practicerelated improvements in somatosensory interval discrimination are temporally specific but generalize across skin location, hemisphere, and modality. J Neurosci 18: 1559-1570.

Nagarajan, S.S., Mahncke, H., Salz, T., Tallal, P., Roberts, T. and Merzenich, M.M. (1999). Cortical auditory signal processing in poor readers. PNAS 96: 6483-6488.

Pandya, P., Moucha, R., Engineer, N.D., Rathbun, R.L., Vazquez, J. and Kilgard, M.P. (2005). Asynchronous inputs alter excitability, spike timing, and topography in primary auditory cortex. Hear Res, 203: 10-20.

Recanzone, G.H., Merzenich, M.M. and Schreiner, C.E. (1992). Changes in the distributed temporal response properties of SI cortical neurons reflect improvements in performance on a temporally based tactile discrimination task. J Neurophysiol 67: 1071-1091.

Schreiner, C.E., Mendelson, J., Raggio, M.W., Brosch, M. and Krueger, K. (1997). Temporal processing in cat primary auditory cortex. Acta Otolaryngol Suppl 532: 54-60.

Siegel, C., Waldo, M., Mizner, G., Adler, L.E. and Freedman, R. (1984). Deficits in sensory gating in schizophrenic patients and their relatives. Arch Gen Psychiatry 41: 607612.

Syka, J. (2002). Plastic changes in the central auditory system after hearing loss, restoration of function, and during learning. Physiol Rev 82: 601-636.

Tallal, P., Merzenich, M.M, Miller, S. and Jenkins,W. (1998). Language learning impairments: integrating basic science, technology, and remediation. Exp Brain Res 123: 210-219. 
Tallal, P., Miller, S.L, Bedi, G., Byma, G., Wang, X., Nagarajan, S.S., Schreiner, C., Jenkins, W.M. and Merzenich, M.M. (1996). Language comprehension in language-learning impaired children improved with acoustically modified speech. Science 271: 81-84.

Tan, A.Y., Zhang, L.I., Merzenich, M.M. and Schreiner, C.E. (2004). Tone-evoked excitatory and inhibitory synaptic conductances of primary auditory cortex neurons. $J$ Neurophysiol 92: 630-643.

Tremblay, K., Kraus, N., McGee, T., Ponton, C. and Otis, B. (2001). Central auditory plasticity: changes in the N1-P2 complex after speech-sound training. Ear Hear 22: 79-90.

van Praag, H., Kempermann, G. and Gage, F.H. (2000). Neural consequences of environmental enrichment. Nat Rev Neurosci 1: 191-198.

van Praag, H., Kempermann, G. and Gage, F.H. (1999). Running increases cell proliferation and neurogenesis in the adult mouse dentate gyrus. Nat Neurosci 2: 266-270.

Warrier, C.M., Johnson, K.L., Hayes, E.A., Nicol, T.G. and Kraus, N. (2004).Learning impaired children exhibit timing deficits and training-related improvements in auditory cortical responses to speech in noise. Exp Brain Res 157:431-41.

Wehr, M. and Zador, A.M. (2003). Balanced inhibition underlies tuning and sharpens spike timing in auditory cortex. Nature 426: 442-446.

Wehr, M. and Zador, A.M. (2005). Synaptic mechanisms of forward suppression in rat auditory cortex. Neuron. 47:437-45.

Wetzel, W., Ohl, F.W., Wagner, T. and Scheich, H. (1998). Right auditory cortex lesion in Mongolian gerbils impairs discrimination of rising and falling frequency-modulated tones. Neurosci Lett. 252:115-8. 


\section{CHAPTER 4}

\section{A BRIEF REVIEW OF 192 IgG-SAPORIN}

Nucleus basalis neurons, which release the neurotransmitter acetylcholine, project to and receive inputs from the amygdala, and are activated as a function of the behavioral significance of stimuli (Figure 4.1; Mesulam et al., 1983; Richardson and DeLong, 1991). Pairing basal forebrain stimulation with sensory stimuli facilitates cortical plasticity (Kilgard and Merzenich, 1998a; 1998b). These results suggest that the temporal correlation of acetylcholine release by nucleus basalis neurons and the occurrence of important events in the environment strengthen the appropriate neural networks required for experiencedependent plasticity.

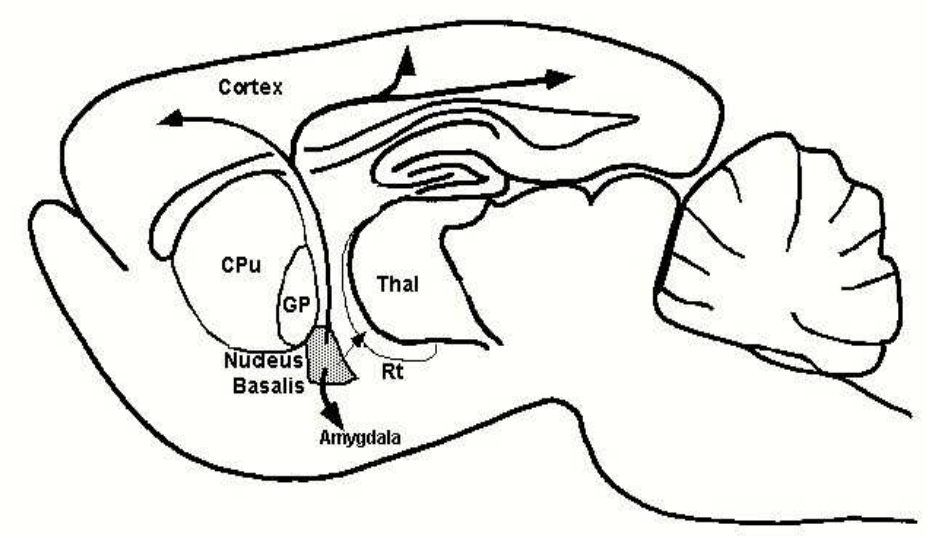

Figure 4.1. Nucleus basalis projections (from Kilgard and Merzenich, 1998b). Nucleus basalis projects to and receives inputs from the amygdala and other limbic structures (Mesulam et al., 1983), and is the major source of cholinergic innervation to the cortex. 
Nucleus basalis lesions prevent plasticity in sensory cortex. For example, the robust cortical reorganization following digit amputation or nerve transaction is prevented by nucleus basalis lesions (Juliano et al., 1991; Webster et al., 1991). However, a major concern of excitotoxic lesion protocols is destruction of cholinergic and noncholinergic neurons in the basal forebrain, as well as damage to adjacent structures (Review Waite and Thal, 1996). Recently, specific neurotoxins have been developed to solve the limitations of excitotoxins. Intracerebroventricular injections of the antibody $192 \mathrm{Ig}-\mathrm{G}$ coupled to the ribosome inactivating protein saporin target p75 nerve growth factor receptor positive (p75NGFr) cells throughout the rat basal forebrain, including the medial septum, vertical, and horizontal diagonal bands of Broca, which project to the hippocampus, and the substantia innominata and nucleus basalis, which project to the cortex (Figure 4.2; Wiley et al., 1991; 1995; Heckers et al., 1994). Nucleus basalis projections to the cortex are homolateral, but 3-7 days after unilateral ventricular injections, the number of cholinergic neurons in the basal forebrain and their choline acetyltransferase and acetylcholinesterase positive terminal fibers can be almost completely reduced due to widespread diffusion through the ventricular system (Heckers et al., 1994; Waite et al., 1994; Johnston et al., 1981). Since deficits are only manifested when there is approximately an $80 \%$ loss of cholinergic markers, the adult rat cholinergic system can tolerate substantial damage (Waite et al., 1995; Wrenn et al., 1999). 
In the adult rat, the optimal dose that produces the maximum depletion of cholinergic markers in the basal forebrain that is accompanied by the smallest amount of non-specific tissue damage is generally 2-2.7 $\mu \mathrm{g}$ (Waite et al., 1995). Cerebellar purkinje neurons express p75NGFr and may be destroyed in the lesion process in a dose-dependent manner, while brain structures, including the amygdala, that do not have p75NTFr cells remain intact following immunotoxic lesions (Heckers et al., 1994; Waite et al., 1995; Walsh et al., 1995). In conclusion, the specificity of immunotoxins is superior to other lesioning methods.

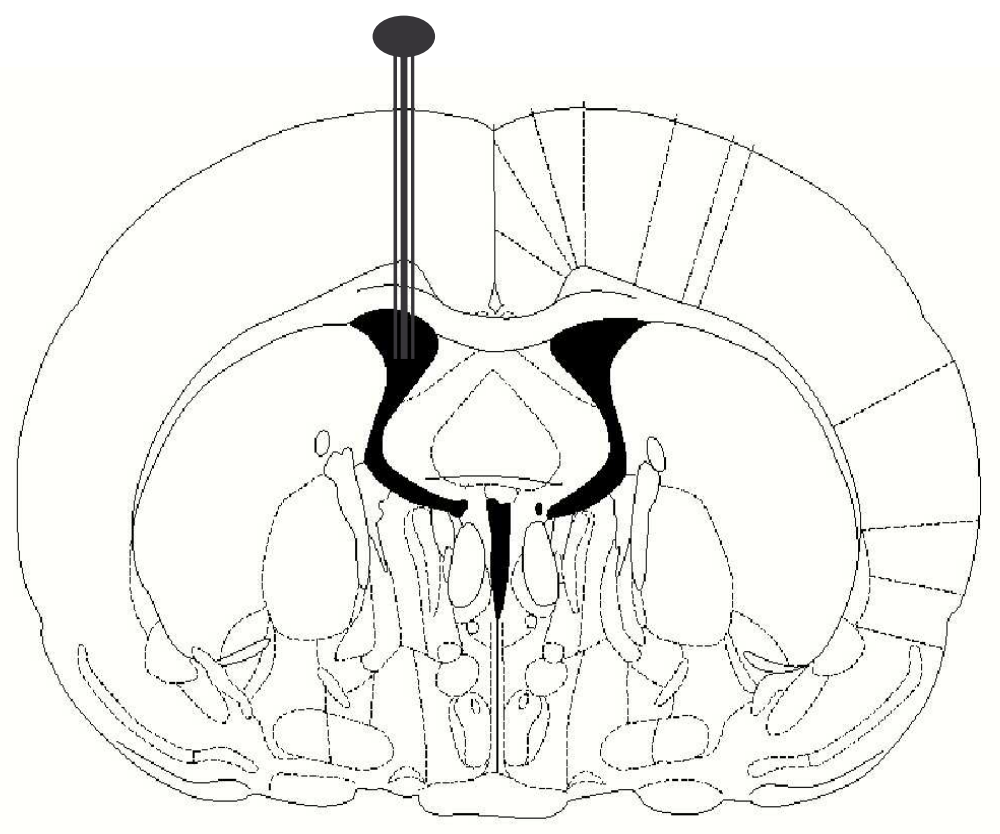

Figure 4.2. Intracerebroventricular injection (modified from Paxinos and Watson 1998). Intracerebroventricular injections of 192 IgG-saporin kill p75 nerve growth factor receptor positive cells throughout the basal forebrain, including the medial septum, vertical, and horizontal diagonal bands of Broca, which project to the hippocampus, and the substantia innominata and nucleus basalis, which project to the cortex (Heckers et al., 1994). 
Selective cholinergic damage impairs experience-dependent plasticity in somatosensory and motor cortex. The reorganization in primary somatosensory cortex after whisker deafferentation is prevented by unilateral injections of 192 IgG-saporin (Baskerville et al., 1997). Cholinergic lesions that block map plasticity in motor cortex impair the acquisition of a motor task (Conner et al., 2003). Cholinergic damage also impairs the ability to learn behaviorally relevant information (Ferreira et al., 2001; Berger-Sweeney et al., 2000; Vale-Martinez et al., 2002). Given that cholinergic damage impairs other robust forms of experience-dependent plasticity, it is possible that enrichment-induced plasticity is also modulated by acetylcholine input from nucleus basalis. Specifically, these results indicate that a unilateral ventricular injection of $2.5 \mu \mathrm{g}$ (a dose sufficient to prevent map plasticity in the auditory cortex-Kilgard and Merzenich, 1998a) may prevent enrichment-induced plasticity in the auditory cortex. 


\section{REFERENCES}

Baskerville, K.A., Schweitzer, J.B. and Herron, P. (1997). Effects of cholinergic depletion on experience-dependent plasticity in the cortex of the rat. Neurosci, 4, 1159-1169.

Berger-Sweeney, J., Stearns, N.A., Frick, K.M., Beard, B. and Baxter M.G. (2000). Cholinergic basal forebrain is critical for social transmission of food preferences. Hippocampus, 10(6), 729-38.

Conner, J.M., Culberson, A., Packowski, C., Chiba, A.A. and Tuszynski, M.H. (2003). Lesions of the basal forebrain cholinergic system impair task acquisition and abolish cortical plasticity associated with motor skill learning. Neuron, 38(5), 819-829.

Ferreira, G, Meurisse, M., Gervais, R, Ravel, N. and Levy, F. (2001). Extensive immunolesions of basal forebrain cholinergic system impair offspring recognition in sheep. Neurosci, 106, 103-115.

Heckers, S., Ohtake, T., Wiley, R.G., Lappi, D.A., Geula, C. and Mesulam, M.M. (1994). Complete and selective cholinergic denervation of rat neocortex and hippocampus but not amygdale by an immunotoxin against p75 NGF receptor. $J$ Neurosci, 14, 12711289.

Johnston, M.V., Young, A.C. and Coyle, J.T. (1981). Laminar distribution of cholinergic markers in neocortex: Effects of lesions. J Neurosci Res, 6, 597-607.

Juliano, S.L., Ma, W. and Eslin, D. (1991). Cholinergic depletion prevents expansion of topographic maps in somatosensory cortex. PNAS, 88, 780-784.

Kilgard, M.P. and Merzenich, M.M. (1998a). Cortical map reorganization enabled by nucleus basalis activity. Science, 279, 1714-1717.

Kilgard, M.P. and Merzenich, M.M. (1998b). Plasticity of temporal information processing in the primary auditory cortex. Nat Neurosci, 1(8), 727-31.

Richardson, R.T. and Delong, M.R. (1991). Electrophysiological studies of the functions of the nucleus basalis in primate. Adv Exp Med Biol 295: 233-52.

Mesulam, M.M., Mufson, E.J., Wainer, B.H. and Levey, A.I. (1983). Central cholinergic pathways in the rat: an overview based on an alternative nomenclature (Ch1-Ch6). Neurosci, 10(4), 1185-201. 
Vale-Martinez, A., Baxter, M.G. and Eichenbaum, H. (2002). Selective lesions of basal forebrain cholinergic neurons produce anterograde and retrograde deficits in a social transmission of food preference task in rats. Eur J Neurosci, 16(6), 983-98.

Waite, J.J. and Thal, L.J. (1996). Lesions of the cholinergic nuclei in the rat basal forebrain: excitotoxins vs. an immunotoxin. Life Sciences, 58(22), 1947-1953.

Waite, J.J., Chen, A.D., Wardlow, M.L., Wiley, R.G., Lappi, D.A. and Thal L.J. (1995). 192 immunoglobulin produces graded behavioral and biochemical changes accompanying the loss of cholinergic neurons of the basal forebrain and cerebellar purkinje cells. Neurosci, 65(2), 463-476.

Waite, J.J., Wardlow, M.L., Chen, A.C., Lappi, D.A., Wiley, R.G. and Thal L.J. (1994). Time-course of cholinergic and monoaminergic changes in rat brain after immunolesioning with 192 IgG-Saporin. Neurosci Lett, 169, 154-158.

Walsh, T.J., Kelly, R.M., Dougherty, K.D., Stackman, R.W., Wiley, R.G. and Kutscher, C.L. (1995). Behavioral and neurobiological alterations induced by the immunotoxin 192 IgG-saporin: cholinergic and noncholinergic effects following ICV injection. Brain Res, 702, 233-45.

Webster, H.H., Hanisch, U.K., Dykes, R.W. and Biesold, D. (1991). Basal forebrain lesions with or without reserpine injection inhibit cortical reorganization in rat hindpaw primary somatosensory cortex following sciatic nerve section. Somatosen and Motor Res, 8(4), 327-346.

Wiley, R.G., Berbos, T.G., Deckwerth, T.L., Johnson, E.M. and Lappi, D.A. (1995). Destruction of the cholinergic basal forebrain using immunotoxin to rat NGF receptor: modeling the cholinergic degeneration of Alzheimer's disease. J Neuro Sci, 128, 157166.

Wiley, R.G., Oeltmann, T.N. and Lappi, D.A. (1991). Immunolesioning: selective destruction of neurons using immunotoxin to rat NGF receptor. Brain Res, 562(1), 149-53.

Wrenn, C.C., Lappi, D.A. and Wiley, R.G. (1999). Threshold relationship between lesion extent and the cholinergic basal forebrain in the rat and working memory impairment in the radial maze. Brain Res, 847(2), 284-98. 


\title{
CHAPTER 5
}

\section{SENSORY EXPERIENCE DETERMINES ENRICHMENT-INDUCED PLASTICITY IN RAT AUDITORY CORTEX}

\author{
Cherie R. Percaccio, Autumn L. Pruette, Shilpa T. Mistry, Yeting H. Chen, \\ Michael P. Kilgard \\ School of Behavioral and Brain Sciences, GR41
}

The University of Texas at Dallas

P.O. Box 830688

Richardson, Texas 75083

Keywords: activity-dependent, acetylcholine, attention, autism, schizophrenia, dyslexia 


\section{ACKNOWLEDGEMENTS}

Cherie R. Percaccio is the primary author of these experiments. Author Autumn L. Pruette wrote the computer programs necessary to analyze the data. Authors Shilpa T. Mistry and Yeting H. Chen assisted Cherie with evoked potential recording sessions and histology collection. The authors thank Sharmin Hossain, Pradeep Kilambi, Eric Kildebeck, Ankur Patel, Sophie Rutenbar, and Janne Yanagi for assistance with evoked potential recording sessions and animal colony management. The authors thank Chris Heydrick for assistance with histology and histochemistry. The authors also thank Vikram Jakkamsetti and Kevin Chang for technical assistance. We would like to extend special recognition to Drs. Lawrence Cauller and Theresa Jones for generously sharing their histological expertise and laboratory equipment. This work was supported by the Cure Autism Now Foundation. 


\section{ABSTRACT}

Environmental enrichment generates anatomical, neurochemical, and physiological plasticity. Our previous studies demonstrated that only a few days of enriched housing increases response strength and paired-pulse depression in the auditory cortex of awake and anesthetized rats (Engineer et al., 2004; Percaccio et al., 2005). Multiple environmental and neurochemical factors likely contribute to the expression of this plasticity. In the current study, we examined the contribution of social stimulation, exercise, auditory exposure, and cholinergic modulation. We recorded epidural evoked potentials from awake rats in response to tone pairs and noise bursts. Neither social stimulation nor exercise experienced in isolation increased response strength; however, paired-pulse depression was greater in grouphoused rats. Cholinergic damage caused by a unilateral intracerebroventricular injection of 192 IgG-saporin did not reduce the degree to which enrichment increased response strength and forward masking. Rats that could hear the enriched environment, but not interact with it, exhibited enhanced responses to tones and increased paired-pulse depression. However, the change in temporal processing and responses to noise bursts were smaller compared to rats actually housed in the enriched environment. These results indicate that while sensory experience is a critical factor modulating cortical plasticity, complete environmental enrichment results in greater plasticity than sensory exposure alone. 


\section{INTRODUCTION}

Environmental enrichment increases social interactions, physical exercise, and sensory stimulation. Over the past 50 years, enriched environments have been used to demonstrate that the structure, chemical composition, and function of the entire brain can change across the lifespan (van Praag et al., 2000; Diamond, 2001). Animals housed in enriched conditions exhibit increases in brain weight, cortical thickness, glial cell to neuron ratio, dendritic branching, number of synapses per neuron, and levels of neurotrophins compared to animals housed in standard laboratory conditions (Diamond et al., 1966;

Bennett et al., 1969; Greenough et al., 1973; Katz and Davies, 1984; Turner and Greenough, 1985; Ickes et al., 2000). Enrichment also increases the levels of acetylcholine (ACh) receptors, acetylcholinesterase (AChE), choline acetyltransferase, and monoamines in multiple brain regions (Bennett et al., 1964; Por et al., 1982; O'Shea et al., 1983; Park et al., 1992; Naka et al., 2002). The anatomical and neurochemical plasticity associated with enriched housing enhances recovery from several forms of brain damage (Johansson, 2003). Enrichment increases the responsiveness of cortical neurons to tactile, visual, and auditory stimuli (Beaulieu and Cynader, 1990a; Coq and Xerri, 1998; Engineer et al., 2004). For example, enrichment dramatically increases the strength of cortical responses to tones and noise bursts in both young and adult rats (Engineer et al., 2004). Enrichment also alters temporal processing in sensory cortex (Beaulieu and Cynader, 1990b; Percaccio et al., 2005). In rat auditory cortex (AC), paired-pulsed depression (PPD) increases during environmental enrichment and returns to normal levels when housed in standard conditions 
(Percaccio et al., 2005). Collectively, these results indicate that environmental enrichment has a profound effect on the form and function of cortical circuits.

Given the complexity of enriched environments, it is possible that many environmental factors stimulate and influence the expression of cortical plasticity. For example, the increased level of physical activity in enriched rats could contribute to neural plasticity. Wheel running is associated with increases in thickness of motor cortex, angiogenesis in both cerebellar and motor cortex, neurogenesis, neurotrophin levels, dopamine, long-term potentiation, and resistance to injury (de Castro and Duncan, 1985; Black et al., 1990; Stummer et al., 1994; Neeper et al., 1996; van Praag et al., 1999; Anderson et al., 2002; Swain et al., 2003; Farmer et al., 2004). Group housing significantly increases cortical weight, neurotrophic factor levels, neuronal density, and behavioral recovery after brain injuries, while isolation rearing results in neuroanatomical, neurochemical, physiological, and behavioral abnormalities (Rosenzweig et al., 1978; Einon et al., 1981; Turner and Greenough, 1985; Geyer et al., 1993; Risedal et al., 2002; Gordon et al., 2003; Preece et al., 2004). Although exercise or social stimulation can generate many of the benefits that accompany general enrichment, it is not known whether they are sufficient to increase response strength and PPD in AC.

Several studies indicate that cortical plasticity is regulated by the behavioral relevance of environmental stimuli. Rats that observe, but do not interact with, an enriched environment do not exhibit increased brain weight and exploratory behavior typical of rats housed in the enriched environment (Ferchmin and Bennett, 1975). Monkeys exposed to sensory inputs without behavioral meaning do not exhibit the cortical map plasticity observed 
in monkeys who use stimuli to make behavioral judgments (Recanzone et al., 1993). Nucleus basalis (NB) neurons, which provide the major source of cholinergic innervation to the cortex, are activated as a function of the behavioral importance of environmental stimuli (Richardson and DeLong, 1991). Cholinergic modulation is necessary for the acquisition of behaviorally relevant information (Berger-Sweeney et al., 2000; Ferriera et al., 2001; Kudoh et al., 2004). Cholinergic receptor antagonists and NB lesions prevent experiencedependent and injury-induced plasticity in auditory, visual, and somatosensory cortices (Sato et al., 1987; Mayo et al., 1988; Metherate and Weinberger, 1989; Delacour et al., 1990; Juliano et al., 1990, 1991; Webster et al., 1991; Gu and Singer, 1993; Ivliev, 1999). ACh application and electrical activation of NB facilitate cortical plasticity when repeatedly paired with sensory stimuli (Metherate et al., 1987; Metherate and Weinberger, 1989; Kilgard and Merzenich, 1998a, b; Verdier and Dykes, 2001). These results suggest that cholinergic neurons may be required for enrichment-induced plasticity.

Collectively, these studies indicate that physical activity, social interaction, and the behavioral relevance of sensory events could influence the expression of enrichment-induced plasticity. In the present study we used chronic awake recordings to document the influence of exercise, social stimulation, auditory experience, and cholinergic modulation on AC responses. 


\section{MATERIALS AND METHODS Environmental Conditions}

Rats in all housing conditions were on a reversed 12-hr light/dark cycle and heard the sounds of room traffic, feeding, and cleaning while they were most active. Constant temperature and humidity were maintained and food and water were provided ad libitum for all rats. All the rats used in this study were female and housed with littermates at Charles River Laboratories until approximately 12 weeks of age. The standard and enriched environmental conditions are identical to those described in our previous studies (Engineer et al., 2004; Percaccio et al., 2005).

\section{Standard Environment}

Rats in the standard environment were housed 1 per cage $(25 \mathrm{~L} \mathrm{x} 15 \mathrm{~W}$ x $18 \mathrm{H} \mathrm{cm}$; Figure $5.1 \mathrm{~A}$ ) in a colony rack of 30 cages. The acoustic environment of this condition consisted of cage noises and vocalizations from 20-30 other rats housed in the same room. Exercise Environment

Rats in the exercise environment were housed 1 per cage $(61 \mathrm{~L}$ x $41 \mathrm{~W}$ x $36 \mathrm{H} \mathrm{cm}$; Figure 5.1 B) with a running wheel (34.5 in circumference) in the same room as the standardhoused rats. On average, rats in this condition ran $1 / 2$ mile per day (1023 revolutions).

\section{Social Environment}

Rats in the social environment were housed 4 per cage $(61 \mathrm{~L}$ x $41 \mathrm{~W}$ x $36 \mathrm{H} \mathrm{cm}$; Figure 5.1 C) in the same room as the standard-housed rats. 


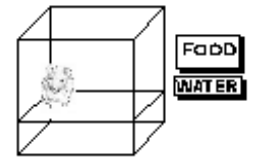

B. EXERCISE ENVIRONMENT

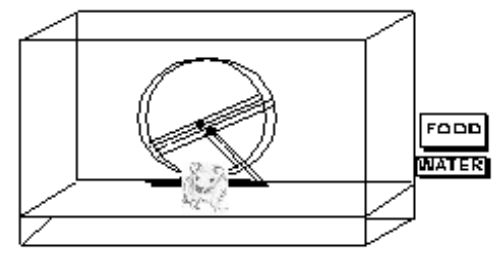

C. SOCIAL ENVIRONMENT

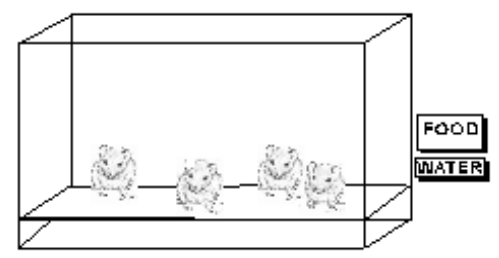

D. ENRICHED ENVIR ONMENT E. AUDITOR Y EXPOSURE ENVIR ONMENT
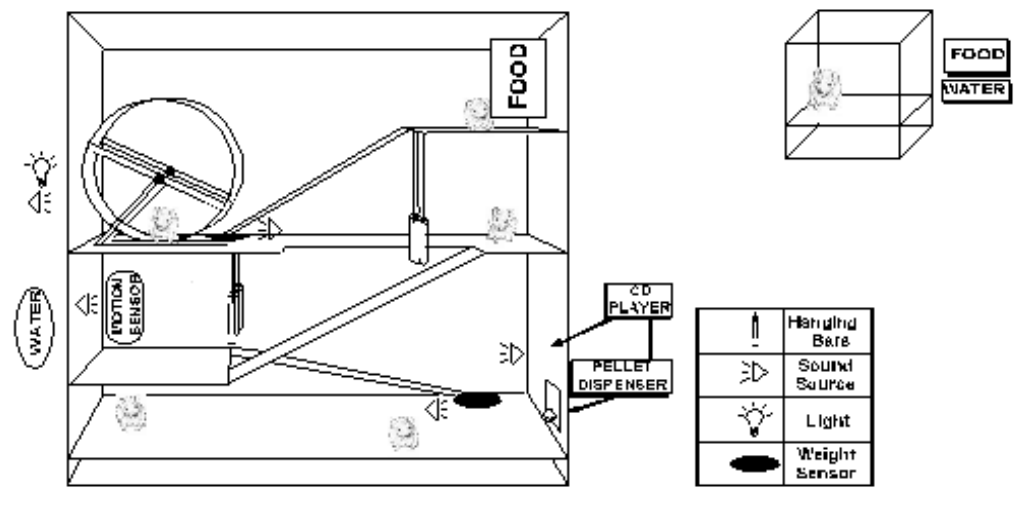

Figure 5.1. Schematics of the five environmental housing conditions. A) 1 rat was housed in the standard environment. B) 1 rat was housed with a wheel in the exercise environment. C) 4 rats were housed together in the social condition. The acoustic environment of these conditions included cage noises and vocalizations from 20-30 other rats also housed in the main rat colony room. D) The enriched environment consisted of 4-8 rats housed in a large cage with devices that generated different sounds when rats crossed a motion detector path, stepped on weight sensors, or passed through hanging bars. Each rotation of the running wheel triggered a brief tone and light flash. A CD player played 74 sounds, including tones, noise bursts, musical sequences and other complex sounds in random order. Some of these sounds were associated with delivery of a sugar reward. E) In the auditory exposure condition, 1 rat was housed in a hanging cage in the same room as the enriched environment. 
Enriched Environment

In the enriched environment, 4-8 rats were housed together in a large cage $(76 \mathrm{~L} \mathrm{x} 45$ W x $90 \mathrm{H} \mathrm{cm}$ ) with 4 levels connected by ramps in a separate room from the standard rat colony (Figure 5.1 D). Touch plates at the bottom of 2 ramps triggered different tones (2100 or $4000 \mathrm{~Hz}$ ) when rats stepped on the plates. Chains, wind chimes, and bells were hung across the entrance of each ramp so that a unique sound was elicited when rats passed from 1 level to the next. A motion detector emitted an electronic chime each time a rat crossed the infrared beam in front of the water source. An exercise wheel emitted a tone $(3000 \mathrm{~Hz}$ Piezo Speaker) and activated a small green light emitting diode with each rotation. Each movement-triggered sound had unique spectral and temporal characteristics that provided behaviorally meaningful information about the location and activity of other rats in the cage.

A CD player presented randomly selected sounds every 2 to 60 seconds, including simple tones, amplitude modulated and frequency modulated tones, noise bursts and other complex sounds (rat vocalizations, classical music, rustling leaves, etc.). Seven of the 74 sounds activated a dispenser (Med Associates) that delivered a sugar pellet to encourage attention to the sounds. The rewarded tracks included interleaved tones of different carrier frequencies $(25 \mathrm{~ms}$ tones of $4,5,9,12,14$, and $19-\mathrm{kHz}$ with interstimulus intervals ranging from $50 \mathrm{~ms}$ to $2 \mathrm{sec}$ ) and frequency modulated sweeps (1 octave up or down in a 140 or 300 ms sweep with interstimulus intervals ranging from 80 to $800 \mathrm{~ms}$ ).

The power spectrums of sounds in the enriched environment spanned the entire hearing range of the rat $(1-45 \mathrm{kHz})$, and were less than $75 \mathrm{~dB}$ SPL. The sound sources added 
to the enriched environment were provided 24 hrs a day and were designed to be more diverse and more behaviorally relevant than sounds in the standard condition. Our definition of a complex sound was any sound louder than $50 \mathrm{~dB}$ SPL with more than one onset separated by no more than $300 \mathrm{~ms}$. The average interval between the components of complex sounds in the enriched environment was significantly shorter compared to the standard environment ( $108 \pm 73$ vs. $133 \pm 80 \mathrm{~ms}$, mean \pm std, $\mathrm{p}<.001)$. The total number of sounds $(>50$ dB SPL) was not significantly different between the enriched and standard environments (23 \pm 23 vs. $19 \pm 15$ sound onsets per minute, mean \pm std).

The enriched environment used in our experiments was designed to increase sensory exposure, social stimulation, and physical activity compared to standard-housed rats (Engineer et al., 2004; Percaccio et al., 2005). In addition to being housed in a large group, a vasectomized male rat was introduced into the cage to encourage natural social interactions, since rats reach sexual maturation by 13 weeks of age (Lewis et al., 2002). We estimate that each rat housed in the enrichment cage ran one fifth of a mile per day (360 rotations).

Although the additional exercise due to movements up and down the levels of the large cage was not quantified, we estimate that rats in the enriched and exercise groups received approximately the same amount of physical activity. In this study, we determine the relative contributions of sensory exposure, social stimulation, and physical activity to enrichmentinduced plasticity. 
Auditory Exposure

Rats were housed 1 per cage (25 L x $15 \mathrm{~W}$ x $18 \mathrm{H} \mathrm{cm}$; Figure $5.1 \mathrm{E})$ in a colony rack in the same room as the enriched environment. The acoustic environment of rats in this condition consisted of sounds described as part of the enriched environment and vocalizations from enriched rats and other singly housed rats in the rack. Although the intensity of the sounds generated by the enriched environment were 5-20 dB quieter for rats in the auditory exposure group, most sounds were audible from any location in the room.

We observed no evidence that any of the housing conditions caused excessive stress (i.e. excessive licking, hair loss, fighting, etc.). Rats actively explored the environment, voluntarily activated sound sources, and engaged in playful behavior. In addition, our behavioral observations and EEG recordings indicate that the different housing conditions did not interfere with normal sleep-wake behavior.

\section{Surgery}

\section{Chronic Implantation}

Eighty-one adult female Sprague-Dawley rats were chronically implanted with a ball electrode over left A1 and a ground screw over the cerebellum. Surgical anesthesia was induced with sodium pentobarbital (50 mg/kg, i.p.). A state of areflexia was maintained throughout the surgery, and supplemental doses of dilute pentobarbital were administered subcutaneously if needed $(0.2 \mathrm{ml} ; 8 \mathrm{mg} / \mathrm{ml})$. Anesthesia level was monitored by response to toe pinch. Atropine $(1 \mathrm{mg} / \mathrm{kg})$ and dexamethasone $(4 \mathrm{mg} / \mathrm{kg})$ were administered subcutaneously to minimize secretions and brain edema during the procedure and recovery. 
Animals received antibiotic injections (ceftriaxone $20 \mathrm{mg} / \mathrm{kg}$ ) before and after surgery. Body temperature was maintained at $37^{\circ} \mathrm{C}$. Four to five structural screws were used to anchor the implant on the skull. The 4-pin connector was held in place with dental acrylic. Fifteen rats were excluded from the experiment due to implant malfunction. Experimental attrition was equal across the 7 groups.

Intracerebroventricular Injection

Cholinergic neurons were selectively lesioned with 192 IgG-saporin (Advanced Targeting Systems). 192 IgG-saporin binds to the neurotrophin p75 low affinity nerve growth factor receptor (p75 NGFr) expressed on cholinergic neurons in the basal forebrain (Wiley et al., 1991, 1995; Kiss et al., 1988). After the immunotoxin is internalized, saporin inhibits protein synthesis (Wiley et al., 1995). Twenty-eight rats received an injection of the active immunotoxin and 27 rats received an injection of unconjugated 192-IgG and saporin. Intracerebroventricular injections (ICV) were made into the left lateral ventricle to target p75 NGFr positive cells in the medial septum, vertical, and horizontal diagonal bands of Broca, which project to the hippocampus, and the substantia innominata and nucleus basalis, which project to the cortex (Heckers et al., 1994). An injection of $2.5 \mathrm{ug}$ of toxin or control (in a volume of $1.7 \mathrm{ul}$ ) was made $0.8 \mathrm{~mm}$ posterior from bregma, $1.4 \mathrm{~mm}$ lateral on the left hemisphere, and $3.8 \mathrm{~mm}$ below the pial surface according to Paxinos and Watson (Paxinos and Watson, 1998). The brain was allowed to settle for 5 minutes prior to injection. The fluid was delivered over 2 minutes, and the brain was allowed to settle 5 minutes following injection. 
EEG electrodes were chronically implanted after the needle was removed. This dose of immunotoxin was sufficient to prevent AC map plasticity induced by NB stimulation (Kilgard and Merzenich, 1998a).

\section{Experimental Time-course}

\section{Environmental Factors Experiment}

Auditory evoked responses were collected from rats in the exercise $(n=7)$, social $(n=7)$, or exposure $(n=6)$ groups during 12 recording sessions (Figure 5.2 A-C). Data was first collected in the standard environment 2 days after chronic implantation of recording electrodes and weekly thereafter. Rats were differentially housed for 8 weeks and then returned to the standard condition for 3 weeks.

\section{Cholinergic Damage Experiment}

In a separate experiment, rats were randomly assigned to 1 of 4 groups: either lesion or sham-enriched, or lesion or sham-standard. Rats in the enriched groups (lesion: $n=11$; sham: $n=11$ ) were injected with 192 IgG-saporin or the inactive control and housed in the standard environment for 13 days, moved to the enriched environment for 35 days, and then returned to the standard environment for 14 days (Figure 5.2 E). Rats in the standard groups (lesion: $\mathrm{n}=12$; sham: $\mathrm{n}=12$ ) were injected with $192 \mathrm{IgG}$-saporin or the inactive control and housed singly in the standard environment for 62 days (Figure 5.2 D). Evoked potential data were collected the day after implantation and every 3-4 days thereafter. Data from the first 13 days after implantation were not included in our analysis since the effects of the lesion develop over this time (Book et al., 1992; Waite et al., 1994; Motooka et al., 2001). 


\section{Age (weeks)}

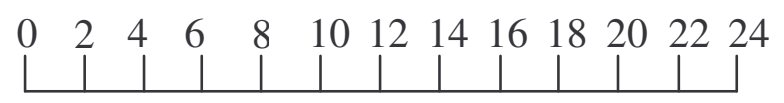
A) Exercise Environment
$N=7$
B) Social Environment

$$
N=7
$$
C) Auditory Environment $N=6$
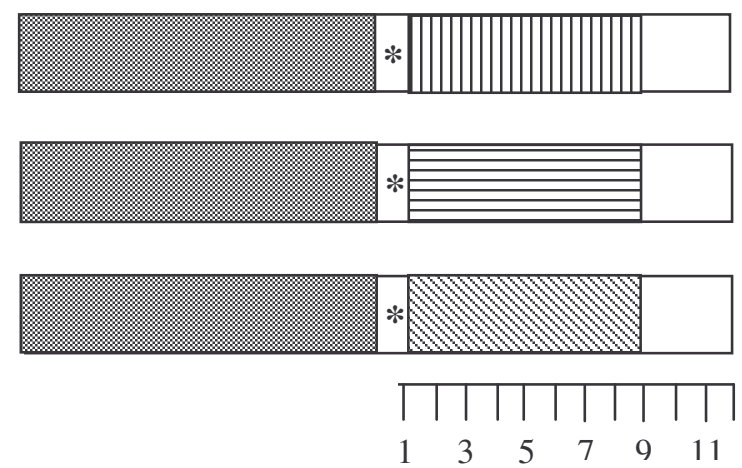

Recording Session

D) Standard

Lesion $N=12$

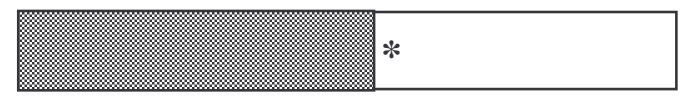

Sham $N=12$

E) Enriched Environment

Lesion $N=11$

Sham $N=11$

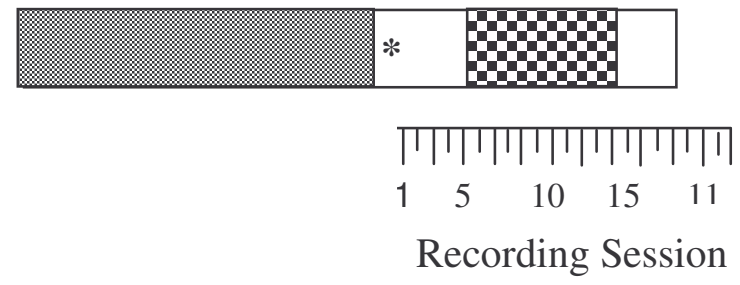

Housed w/ cage
mates

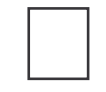

Standard

Environment

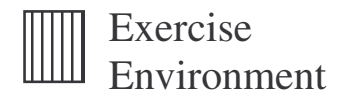

Social

Environment

Auditory

Environment
Enriched

Environment

Figure 5.2. Experimental time-lines. Asterisks indicate chronic electrode implantation. A-C) Evoked potential data were collected over 12 recording sessions. Data was first collected in the standard environment 2 days after chronic implantation of recording electrodes. Rats were differentially housed for 8 weeks and then returned to the standard condition for 3 weeks. D-E) Evoked potential data were collected the day after implantation and every 3-4 days thereafter for 62 days from each rat in the standard and enriched environments. Rats in the standard groups were injected with $192 \mathrm{IgG}$-saporin or the inactive control and housed in the standard environment for 62 days. Following injection, rats in the enriched groups were housed in the standard environment for 13 days, moved to the enriched environment for 35 days, and then returned to the standard environment for 14 days. 


\section{Stimulus Presentation}

Recordings were made during the dark cycle in all housing conditions to encourage rats to be as alert as possible. However, the rats did spend some time sleeping. EEG recordings indicate that rats were in slow wave sleep no more than $25 \%$ of the time during each recording session. Overall, no differences in activity level, exploration, time spent sleeping, or arousal levels were noted across experimental groups during recording sessions.

Middle latency evoked potential data were collected from each rat in a soundattenuated booth. Acoustic stimuli included pairs of $25 \mathrm{~ms} 9-\mathrm{kHz}$ tones with interstimulus intervals of either 500, 200, 100, or $50 \mathrm{~ms}$ (70 dB SPL with $3 \mathrm{~ms}$ rise and fall times), and 3 different 100 ms long noise bursts (loud: 70 dB SPL with 3 ms rise and fall times; quiet: 50 dB SPL with 3 ms rise and fall times; ramped: $70 \mathrm{~dB}$ SPL with $50 \mathrm{~ms}$ rise and fall times). Tones and noises were presented from a speaker centered above the cage and randomly interleaved every 10 s. Signals were low-pass filtered $(800 \mathrm{~Hz})$, amplified (10,000X), and displayed on an oscilloscope for monitoring. Data acquisition computers collected and averaged cortical responses to 125 presentations of each stimulus. Trials with excessive motion artifacts $(>0.1 \mathrm{mV})$ were discarded prior to analysis of the mean evoked potential.

\section{Histotechniques}

\section{Histology}

Rats were sacrificed in groups of 3 (1 lesioned rat, 1 sham rat, and 1 uninjected rat from the exercise, social, or exposure group), and their brain sections processed simultaneously to minimize variability. All solutions were warmed to room temperature prior 
to perfusion to increase penetration rate and reduce postmortem autolysis. Rats were overdosed with urethane and transcardially perfused with $100 \mathrm{ml}$ of $0.9 \% \mathrm{NaCl}$ and $0.5 \%$ sodium nitrate in $0.01 \mathrm{M}$ phosphate buffer $(\mathrm{pH} 7.4)$ followed by $200-400 \mathrm{ml}$ of $4 \%$ paraformaldehyde in $0.1 \mathrm{M}$ phosphate buffer ( $\mathrm{pH} 7.4$ ) and $100 \mathrm{ml}$ of $10 \%$ sucrose in $0.1 \mathrm{M}$ phosphate buffer. Brains were removed, post-fixed for 24 hours in $4 \%$ paraformaldehyde, and stored in $30 \%$ sucrose for 48 hours. Brains were sectioned 50 microns thick on a freezing microtome into $0.1 \mathrm{M}$ phosphate buffer. Free-floating sections were stored overnight in a refrigerator.

\section{Histochemistry}

AChE stained sections were examined to determine the extent of cortical cholinergic denervation. The staining protocol was modified from Karnovsky and Roots by Dr. Lawrence Cauller at the University of Texas at Dallas (Karnovsky and Roots, 1964). All solutions were made fresh each time and filtered prior to use. Free-floating sections were agitated throughout the procedure to ensure adequate exposure to each bath of solution. The phosphate buffer ( $\mathrm{pH}$ 7.4) was replaced with $0.1 \mathrm{M}$ sodium acetate buffer ( $\mathrm{pH}$ 6.0) in several washes. The pre-incubation solution consisted of $1.47 \mathrm{~g} / \mathrm{ml}$ of sodium citrate, $0.48 \mathrm{~g} / \mathrm{ml}$ of cupric sulfate, and $0.17 \mathrm{~g} / \mathrm{ml}$ of potassium ferricyanide dissolved in $0.1 \mathrm{M}$ sodium acetate buffer. The sections were immersed in separate baths of the pre-incubation solution twice for 10 minutes. Acetylthiocholine iodide $(0.5 \mathrm{~g} / \mathrm{ml})$ and ethopropazine $(0.05 \mathrm{~g} / \mathrm{ml})$ were added to a clean bath of pre-incubation solution of the same batch to create the incubation solution. Sections incubated for 1 hour, were detoxified in $0.05 \mathrm{M}$ Tris buffer ( $\mathrm{pH} 7.4)$, mounted out of 
$10 \%$ phosphate buffer onto gelatin-coated slides, and photographed for subsequent quantitative analysis.

Throughout this study, adequate measures were undertaken to minimize pain or discomfort of rats. All protocols and recording procedures conformed to the Ethical Treatment of Animals (NIH) and were approved by the University Committee on Animal Research at the University of Texas at Dallas.

\section{Analysis}

\section{Response Strength}

To improve the signal to noise ratio, we compared the average of each individual's mean evoked potential for all presentations during the standard housing condition, including before and after differential housing, with all presentations during either the enriched, social, exercise, or exposure conditions. The response to the first tone of the 500 and $200 \mathrm{~ms}$ interstimulus interval tone pairs were averaged (resulting in 250 presentations) to increase the reliability of the evoked response. The root-mean-square of the evoked potential (30-100 ms after sound onset) was used to quantify the magnitude of the auditory evoked response. To eliminate variability in evoked potential amplitude due to individual differences in electrode position and recording characteristics, the size of the average evoked response during periods of differential housing was divided by the average of the periods in standard housing. As in our previous study, changes were quantified using the plasticity index, which is simply the logarithm base 2 of this ratio (such that -1 and +1 indicate 2 -fold decreases and increases, respectively) (Engineer et al., 2004). Paired t-tests were used to determine whether 
differences in evoked potential response strength were statistically significant (alpha=0.01). Unless otherwise noted, error measures in this study represent standard error of the mean. Paired-pulse depression

For each rat the response to the $2^{\text {nd }}$ tone was calculated by subtracting the response to a single tone (derived from the first pulse of the $500 \mathrm{~ms}$ tone pair) from the overlapping response to 2 tones separated by shorter $(200,100$, or $50 \mathrm{~ms})$ interstimulus intervals. We estimated the waveform of the response $500 \mathrm{~ms}$ after a single tone onset by using the waveform $500 \mathrm{~ms}$ after onset of the $50 \mathrm{~ms}$ pair of tones since we did not present the tone in isolation. The waveform was indistinguishable from baseline $500 \mathrm{~ms}$ after onset of the $50 \mathrm{~ms}$ pair. PPD was quantified as the root-mean-square of the evoked potential 10-175 ms after onset of the $2^{\text {nd }}$ tone (N1-P1 complex) divided by the RMS of the $1^{\text {st }}$ tone. Paired t-tests were used to determine whether differences in PPD were statistically significant (alpha=0.01).

\section{Lesion Confirmation}

In each rat, the reduction of AChE staining was used to confirm immunolesions. Sections were imaged in 36-bit color (12-bit RGB) using a high-resolution digital camera (DVC Co., Austin, TX, USA, Mod. 1310/1312) and Neurolucida TM software (Microbrightfield, Inc., Williston, VT). Using Adobe Photoshop (6.0), we quantified AChE optical density (inverse of mean luminosity) across all layers of primary AC. The brightness and contrast settings of all images were held constant during image acquisition and analysis. The intensity of AChE staining was determined as the average of approximately 10 sections through the extent of auditory cortex from each rat to control for local variations in staining 
(Paxinos and Watson, 1998). AChE staining was estimated by subtracting the background staining observed in nearby white matter. Percent of AChE reduction is the intensity of ipsilateral staining divided by the intensity of contralateral staining subtracted from 1. 


\section{RESULTS}

Neurophysiologic responses were recorded from rats housed in environments

designed to determine whether exercise, social stimulation, and sensory exposure contributed to the enrichment-induced plasticity documented in our previous studies (Engineer et al., 2004; Percaccio et al., 2005). Evoked potentials were also recorded from rats with cholinergic or sham lesions housed in standard laboratory conditions or an enriched environment to determine the contribution of ACh to environmental plasticity.

\section{Sham-Enriched}

The morphology of the grand mean average evoked potential of the sham-enriched group was very similar to that documented in our previous studies. The evoked response to a $70 \mathrm{~dB}$ tone consisted of negative peaks $\sim 25(\mathrm{~N} 1 \mathrm{a}), \sim 40(\mathrm{~N} 1 \mathrm{~b})$, and $\sim 160 \mathrm{~ms}(\mathrm{~N} 2)$ after onset and a positive peak $\sim 85 \mathrm{~ms}$ (P1) after sound onset (Figure 5.3 A). We compared the average of each individual's mean evoked potential recorded before, during, and after enrichment. The amplitudes of the N1b, P1, and N2 peaks in the grand mean average response to the tone before enrichment were $-14,24$, and $-18 \mathrm{mV}$, respectively. During enrichment, the amplitudes of the N1b, P1, and N2 peaks increased by 250\%, 136\%, and 37\%, respectively. 


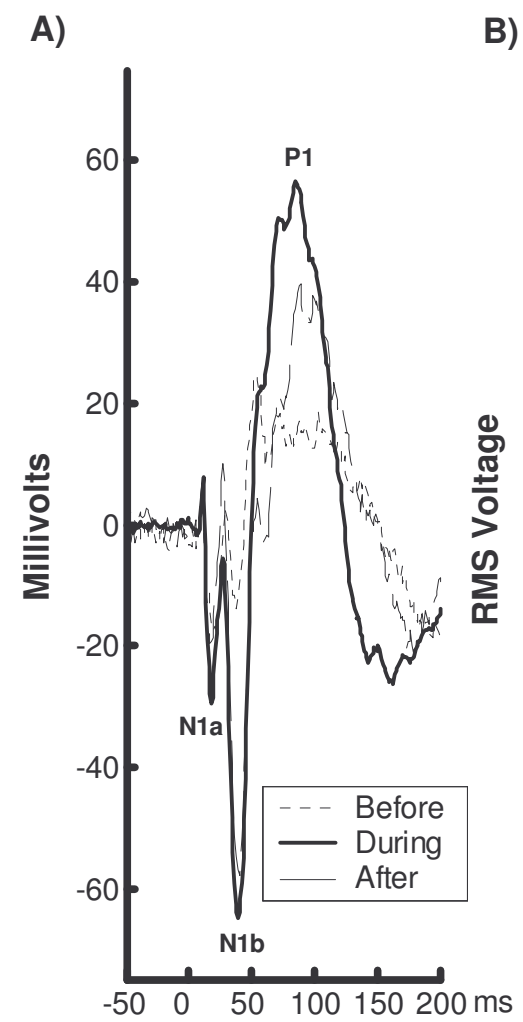

B)

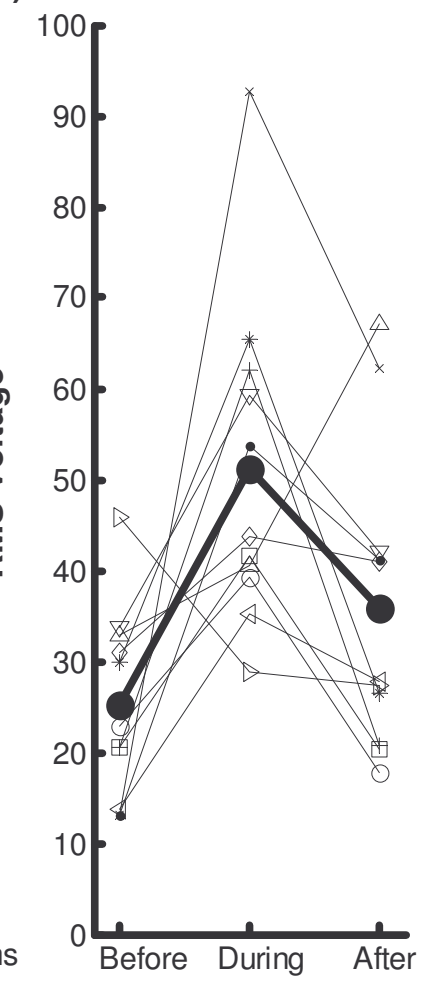

C)

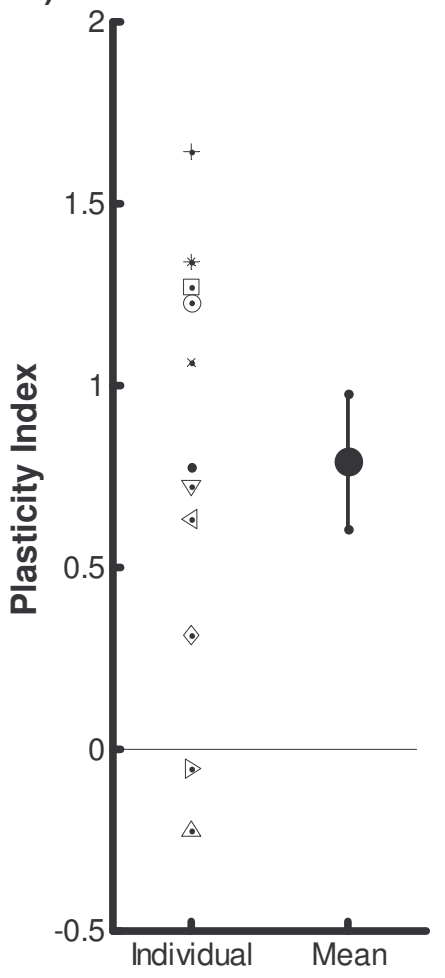

Figure 5.3. Mean auditory evoked potential, grand mean average, and plasticity index of sham-enriched group. A) Mean auditory evoked potential in response to a $70 \mathrm{~dB} 9-\mathrm{kHz}$ tone recorded from an individual rat in the sham-enriched group before, during, and after enrichment. The evoked response to the tone consisted of negative peaks 25 (N1a), 40 (N1b), and $160 \mathrm{~ms}(\mathrm{~N} 2)$ after onset and a positive peak $85 \mathrm{~ms}(\mathrm{P} 1)$ after sound onset. During enrichment, the amplitudes of the N1b, P1, and N2 peaks significantly increased. B) Rootmean-square was used to quantify the power of the average evoked potential in each individual. The mean RMS evoked response of individual rats in the sham-enriched group increased by $73 \%$. C) The plasticity index represents the logarithm base 2 of the average response of each rat during enrichment normalized to the response during housing in the standard environment. A plasticity index value of 1 indicates a 2 -fold increase in the magnitude of the evoked potential compared to week 1 , while a value of -1 would indicate that the magnitude of the evoked potential was halved. The relative changes in tone-evoked plasticity for each individual rat is compared to the mean tone-evoked plasticity $(0.79 \pm 0.19)$ for the sham-enriched group during enrichment. 
Since the shape of an individual's mean evoked response often differed from the grand mean average, we used the root-mean-square of the N1-P1 complex (30-100 ms after tone onset) for each rat to quantify the power of the average evoked potential in each individual. The RMS voltage increased for 10 out of 11 rats when they were moved from the standard to the enriched condition and decreased for 10 out of 11 rats after they were moved from the enriched back to the standard condition (Figure 5.3 B). During enrichment the mean RMS evoked response of individual rats in the sham-enriched group increased by $73 \%$. Each individual's average evoked response during enrichment was divided by the average response while in the standard environment to quantify proportional changes in response strength, and reduce variability due to electrode placement. The relative changes in toneevoked plasticity for each individual rat compared to the mean tone-evoked plasticity for the sham-enriched group are illustrated in Figure 5.3 C. A plasticity index value of 1 indicates a 2-fold increase in the magnitude of the evoked potential compared to week 1, while a value of -1 would indicate that the magnitude of the evoked potential was halved. The average tone-evoked plasticity index was $0.79 \pm 0.19$ (mean \pm s.e.) during enrichment (Figures $5.3 \mathrm{C} \&$ 5.4; Table 5.1). During enrichment, the grand mean average of the tone-evoked responses was significantly larger than during standard housing $(\mathrm{p}<0.05$; Figure 5.4). The response to the $2^{\text {nd }}$ of 2 tones separated by $200 \mathrm{~ms}$ was $36 \pm 2 \%$ of the response to the $1^{\text {st }}$ (Figure 5.5 ). The amount of PPD was greater during enrichment than during standard housing at every interval tested $(\mathrm{p}<0.05$, Table 5.2). Collectively, these results confirm our earlier 
observations that housing in an enriched environment increases response strength and PPD compared to standard-housed rats (Engineer et al., 2004; Percaccio et al., 2005).

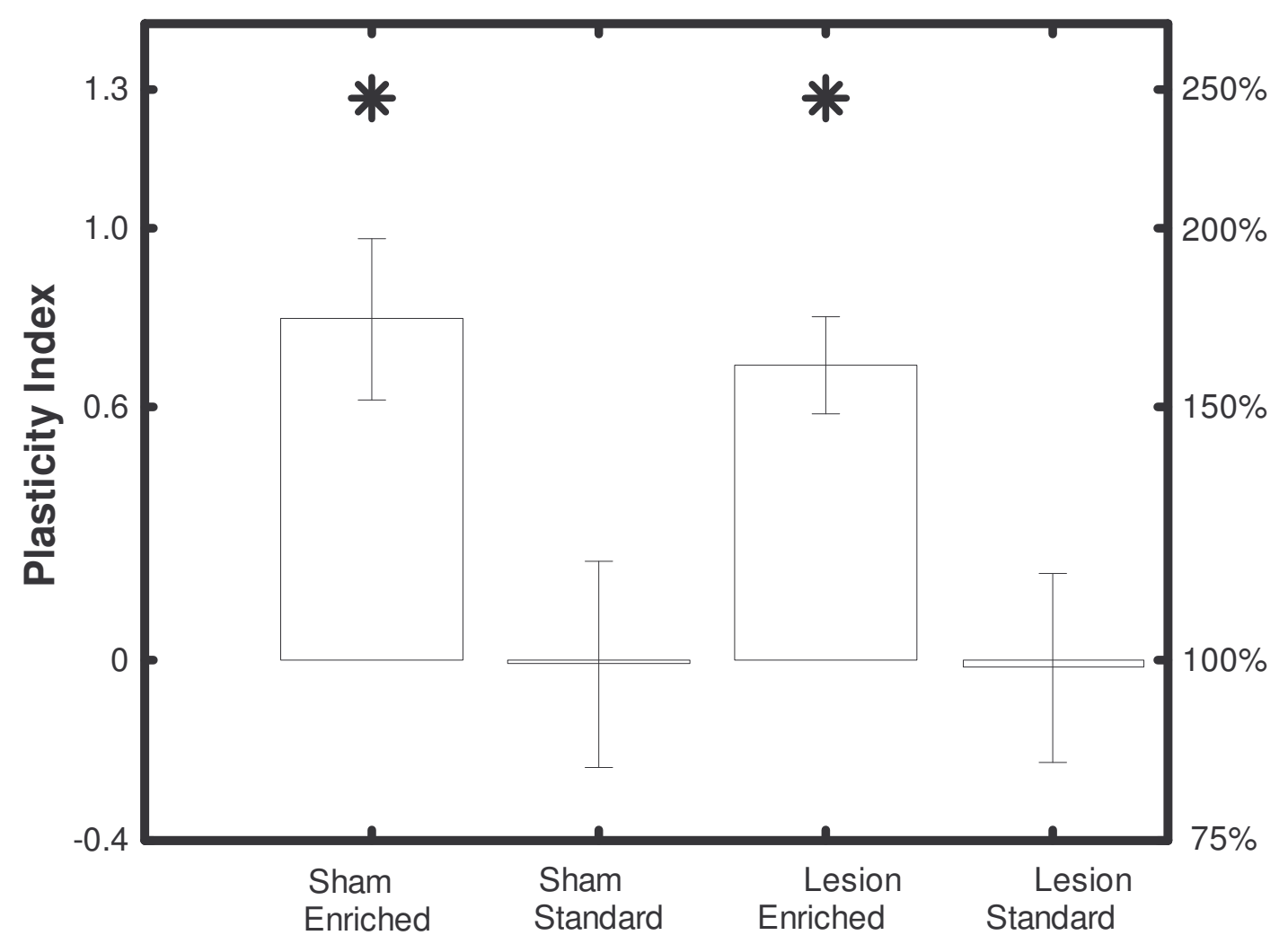

Figure 5.4. Environmental plasticity index for sham-enriched, sham-standard, lesionenriched, and lesion-standard groups. Despite significant damage to the cholinergic input to the cortex, the strengthening of tone-evoked responses occurred to the same extent in both sham and lesion-enriched rats. During enrichment, the grand mean average of the toneevoked responses was significantly larger than in standard-housed rats. Asterisks indicate significant increases in the cortical evoked response $(p<0.05)$. Error bars represent standard error of the mean. 


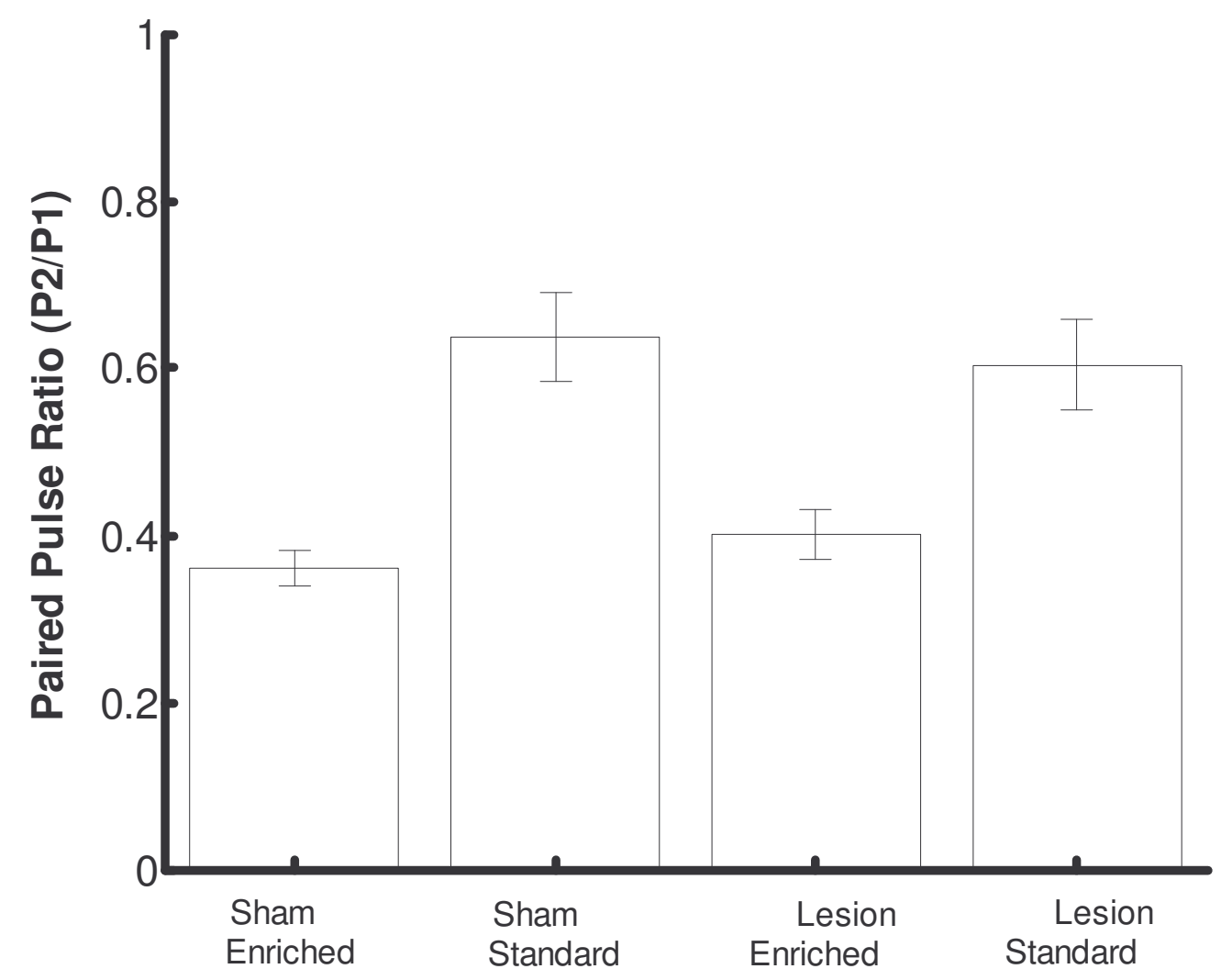

Figure 5.5. Paired-pulse depression (ratio of the RMS to the $2^{\text {nd }}$ pulse divided by the RMS to the $1^{\text {st }}$ pulse) for sham-enriched, sham-standard, lesion-enriched, and lesion-standard groups. PPD significantly increased when rats were housed in the enriched environment compared to standard housing. The response to the $2^{\text {nd }}$ of 2 tones separated by $200 \mathrm{~ms}$ was reduced by $36 \pm 2 \%$ in the sham-enriched group, $64 \pm 5 \%$ in the sham-standard group, $40 \pm 3 \%$ in the lesionenriched group, and $60 \pm 5 \%$ in the lesion-standard group. Error bars represent standard error of the mean. 


\section{GROUPS}

$\begin{array}{lllll} & 500 \mathrm{~ms} & 200 \mathrm{~ms} & 100 \mathrm{~ms} & 50 \mathrm{~ms} \\ \text { Sham-enriched } & 58 \pm 3 \% & 36 \pm 2 \% & 36 \pm 3 \% & 22 \pm 3 \% \\ \text { Sham-standard } & 73 \pm 4 \% & 64 \pm 5 \% & 64 \pm 8 \% & 40 \pm 5 \% \\ \text { Lesion-enriched } & 66 \pm 4 \% & 40 \pm 3 \% & 32 \pm 3 \% & 26 \pm 4 \% \\ \text { Lesion-standard } & 80 \pm 7 \% & 60 \pm 5 \% & 66 \pm 9 \% & 50 \pm 9 \% \\ \text { Exercise } & 81 \pm 2 \% & 71 \pm 5 \% & 66 \pm 4 \% & 54 \pm 4 \% \\ \text { Social } & 78 \pm 5 \% & 53 \pm 3 \% & 57 \pm 5 \% & 45 \pm 6 \% \\ \text { Auditory Exposure } & 68 \pm 2 \% & 44 \pm 2 \% & 42 \pm 6 \% & 32 \pm 2 \%\end{array}$

Table 5.1. Paired-pulse depression (response of $2^{\text {nd }}$ tone/response of $1^{\text {st }}$ tone) values of toneevoked potentials for each experimental group. Values represent mean \pm standard error.

\section{Cholinergic Damage}

Since enrichment increases cholinergic markers, we injected $2.5 \mu \mathrm{g}$ of the immunotoxin 192 IgG-saporin into the left lateral ventricle to determine whether cholinergic inputs from the basal forebrain contribute to this form of cortical plasticity (Bennett et al., 1964; Por et al., 1982; Park et al., 1992). We confirmed cholinergic damage in each animal by staining for $\mathrm{AChE}$, and determined the percent of $\mathrm{AChE}$ reduction by subtracting the intensity of ipsilateral staining from one and dividing by the intensity of contralateral staining (see Figure 5.6 for representative examples). Every lesioned rat exhibited a reduction of cortical AChE staining of at least $25 \%$, while only $16 \%$ of sham lesioned rats and $0 \%$ of 
controls exhibited a reduction of $25 \%$ or more. On average, ipsilateral AChE staining was reduced relative to contralateral AChE staining by $54 \pm 7 \%(\mathrm{p}<0.0001)$ in the lesioned group, by $6 \pm 6 \%$ in the sham group, and by $0 \pm 6 \%$ in the control group. While it is not straightforward to estimate the percent of destruction of cholinergic NB neurons that project to cortex from diffuse AChE staining, previous studies have shown that $192 \mathrm{IgG}$-saporin doses between $2 \& 2.7 \mu \mathrm{g}$ eliminate $60-85 \%$ of choline acetyltransferase and AChE immunoreactive fibers in cortical areas (Waite et al., 1995; Walsh et al., 1995; Galani et al., 2002).

Despite significant damage to the cholinergic input to the cortex, the strengthening of evoked responses occurred to the same extent in both sham- and lesion-enriched rats (Figure 5.4, Table 5.1). Neither sham nor lesion rats exhibited response plasticity when housed in standard conditions. The response to the $2^{\text {nd }}$ of 2 tones separated by 200 ms was $40 \pm 3 \%$ of the response to the $1^{\text {st }}$ (Figure 5.5, lesion-enriched). For lesioned rats, the amount of PPD was greater in enriched rats compared to standard-housed rats at every interval tested $(\mathrm{p}<0.05$, Table 5.2). These results indicate that significant cholinergic damage does not prevent enrichment-induced plasticity in auditory cortex. 
A. Anterior auditory cortex

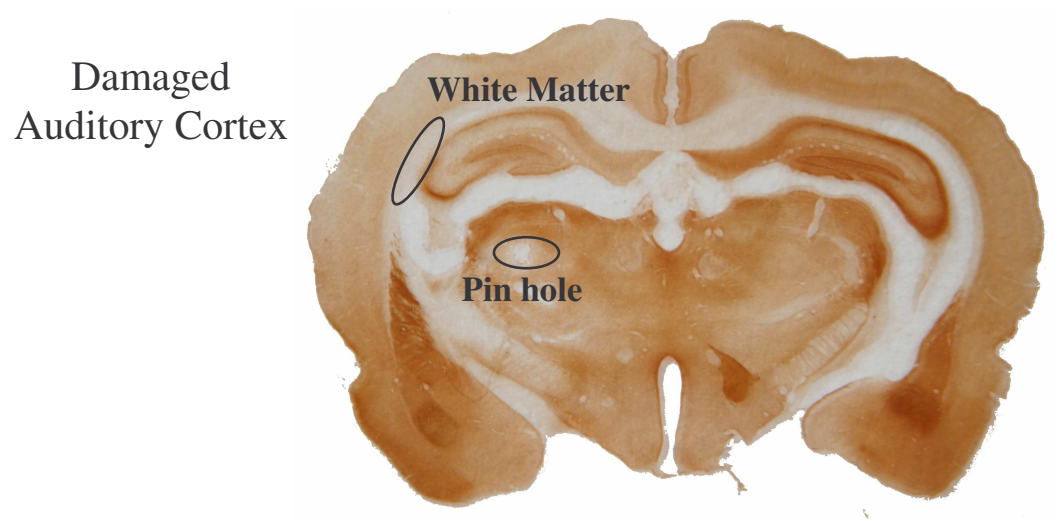

B. Posterior auditory cortex

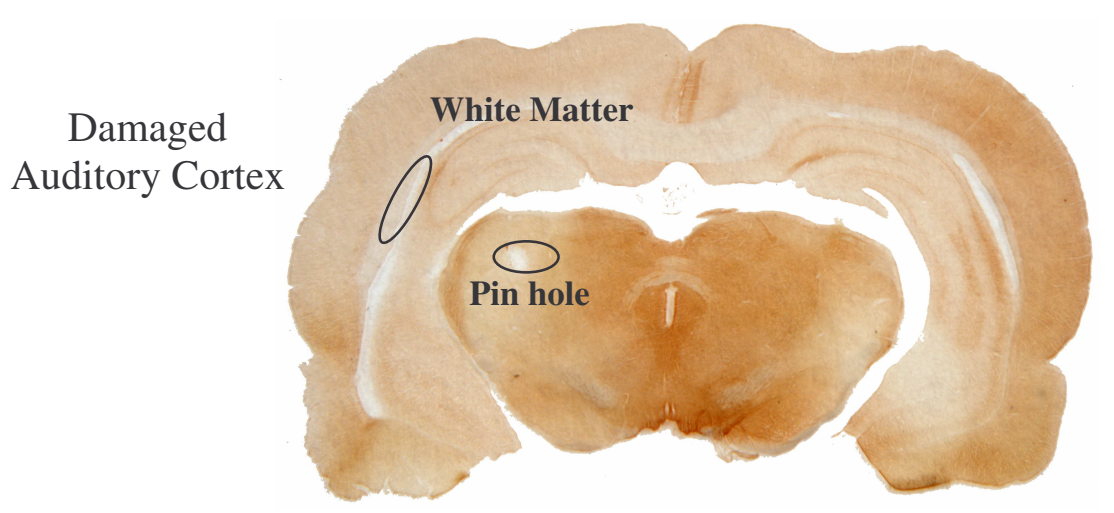

Figure 5.6. Examples of AChE stained sections to verify cholinergic damage. Pin hole indicates left hemisphere. Cholinergic damage extended primarily ipsilaterally throughout auditory cortex. AChE staining was estimated by subtracting the background staining observed in nearby white matter. Percent of AChE reduction is the intensity of ipsilateral staining divided by the intensity of contralateral staining subtracted from 1. A) Anterior section of auditory cortex from rat \#43 and B) Posterior section of auditory cortex from rat $\# 16$. 
GROUP

$\begin{array}{lcccc} & \begin{array}{c}\text { Averaged } \\ \text { Tone }\end{array} & \begin{array}{c}\text { Quiet } \\ \text { Noise Burst }\end{array} & \begin{array}{c}\text { Ramped } \\ \text { Noise Burst }\end{array} & \begin{array}{c}\text { Loud } \\ \text { Noise Burst }\end{array} \\ \text { Sham-enriched } & 0.79 \pm 0.19^{*} & 0.69 \pm 0.16^{*} & 0.55 \pm 0.16^{*} & 0.38 \pm 0.15 \# \\ \text { Sham-standard } & -0.01 \pm 0.24 & -0.01 \pm 0.22 & 0.05 \pm 0.21 & 0.03 \pm 0.19 \\ \text { Lesion-enriched } & 0.68 \pm 0.11^{*} & 0.72 \pm 0.16^{*} & 0.57 \pm 0.12 * & 0.43 \pm 0.14 * \\ \text { Lesion-standard } & -0.02 \pm 0.22 & -0.10 \pm 0.16 & -0.03 \pm 0.15 & -0.04 \pm 0.14 \\ \text { Exercise } & 0.04 \pm 0.24 & -0.20 \pm 0.24 & -0.32 \pm 0.26 & -0.29 \pm 0.25 \\ \text { Social } & 0.14 \pm 0.26 & 0.23 \pm 0.17 & 0.14 \pm 0.17 & 0.17 \pm 0.17 \\ \text { Auditory Exposure } & 1.03 \pm 0.39 \# & 0.49 \pm 0.33 & 0.24 \pm 0.26 & 0.32 \pm 0.27\end{array}$

Table 5.2. Plasticity index values for each experimental group computed from auditory cortex potentials evoked by four different sounds. Values represent mean \pm standard error. Student's $t$-tests were used to determine statistical significance. Asterisks indicate significant differences from zero $\mathrm{p}<0.05$. Pound signs indicate significant difference from zero $\mathrm{p}<0.01$.

\section{Environmental Factors}

To determine what aspects of the enriched environment were responsible for the observed changes in response amplitude and PPD, we recorded the evoked responses of rats housed with an exercise wheel, rats housed in a social environment, and rats that were passively exposed to the sounds of the enriched environment. During the period of differential housing, the average tone-evoked potential amplitude of the auditory exposure group increased 2-fold, while the response of rats in the exercise and social groups were not altered ( $\mathrm{p}<0.001$; Figure 5.7; Table 5.1). PPD was also increased during periods of 
auditory exposure, but, compared to enrichment, was not significantly altered by exercise or social housing (Figure 5.8; Table 5.2). However, rats housed in a social environment had significantly more paired-pulse depression that either standard-housed rats or rats housed with a running wheel. Additionally, the degree of paired-pulse depression that resulted from social stimulation was not different from the degree of paired-pulse depression that resulted from passive auditory stimulation. These result indicate that that there is a continuum of temporal plasticity, but that auditory sensory exposure is the critical factor of the enriched environment that generated the increased amplitude and enhanced PPD documented in our previous studies (Engineer et al., 2004; Percaccio et al., 2005). Although auditory exposure increased response strength as much as full enrichment, PPD was greater in sham-lesioned enriched rats compared to rats that only heard the enriched environment $(\mathrm{p}<0.05$; Table 5.2). In conclusion, housing conditions that increase response magnitude also increase PPD, suggesting that increased PPD may be a consequence of increased response strength (Figure 5.9). 


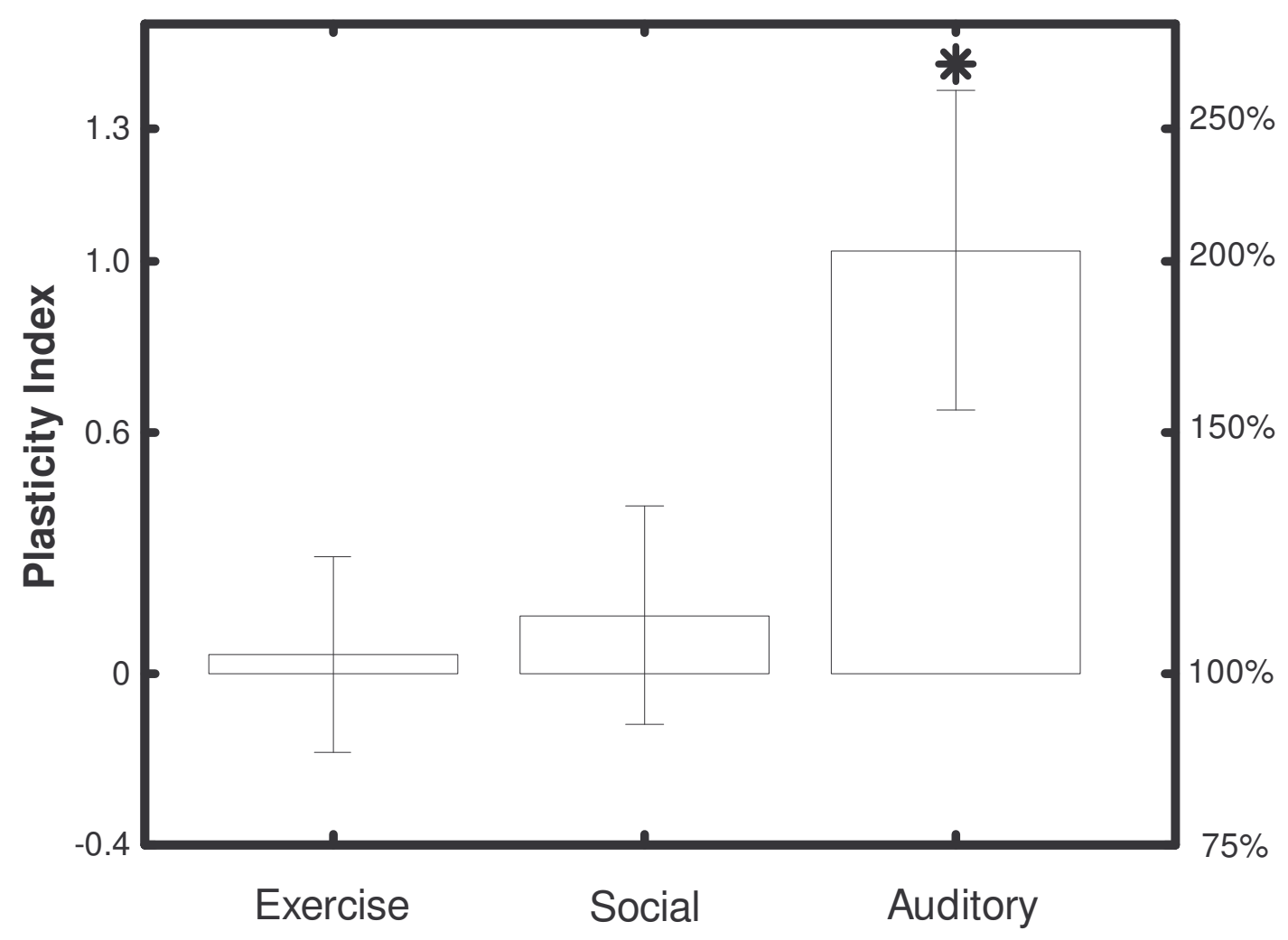

Figure 5.7. Environmental plasticity index for exercise, social, and auditory exposure groups. During the period of differential housing, the average evoked potential amplitude of the auditory exposure group increased 2-fold, while the response of rats in the exercise and social groups were not altered. Asterisks indicate significant differences among the groups $(\mathrm{p}<0.001)$. Error bars represent standard error of the mean. 


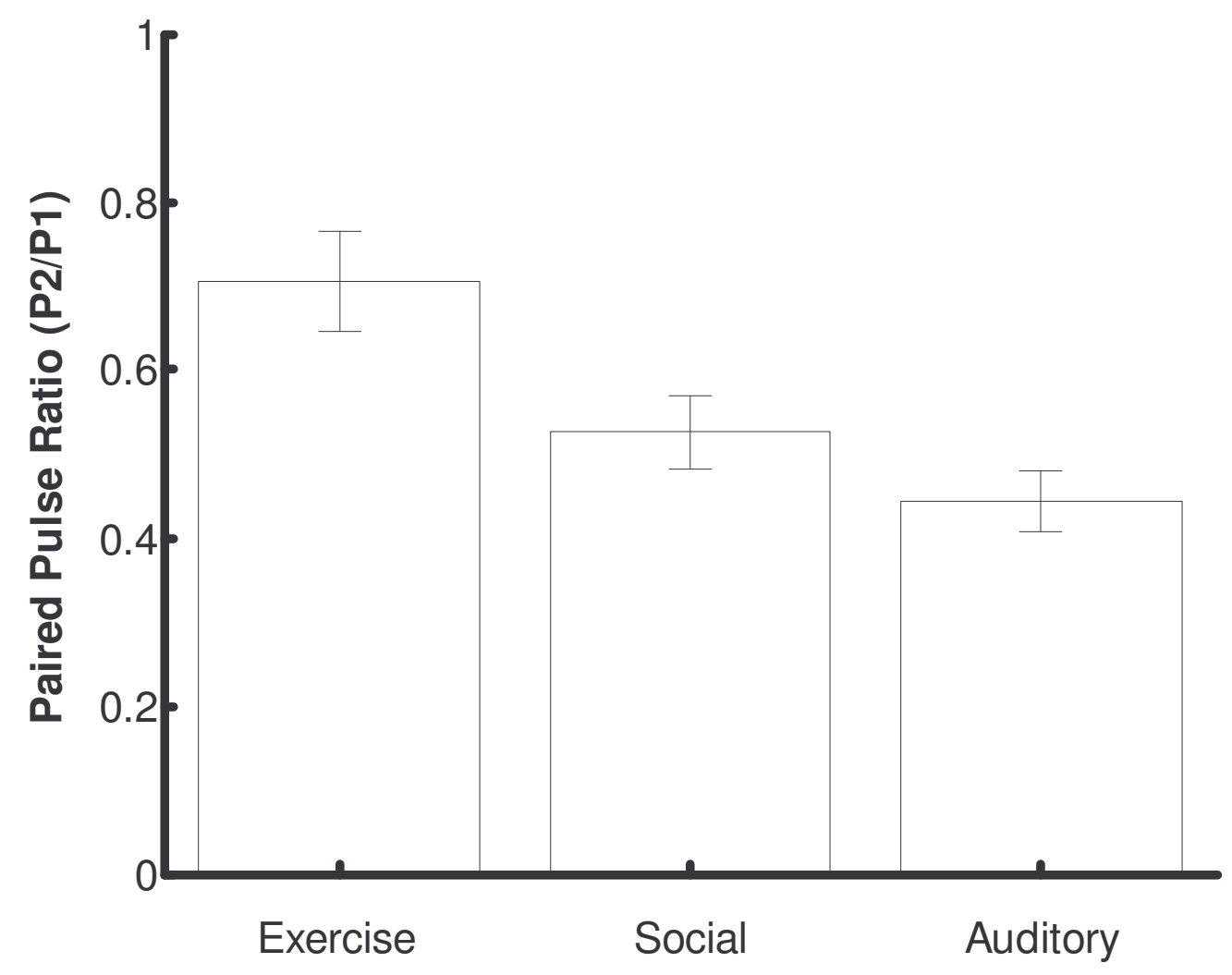

Figure 5.8. PPD was also increased during periods of auditory exposure, but, compared to enrichment, was not significantly altered by exercise or social housing. Social housing increased PPD more than being housed with a running wheel, and was not different than being passively exposed to the sounds associated with the enriched environment. The response to the $2^{\text {nd }}$ of two tones separated by $200 \mathrm{~ms}$ was reduced by $44 \pm 2 \%$ in the auditory exposure group, $71 \pm 5 \%$ in the exercise group, and $53 \pm 3 \%$ in the socially housed group. Error bars represent standard error of the mean. 


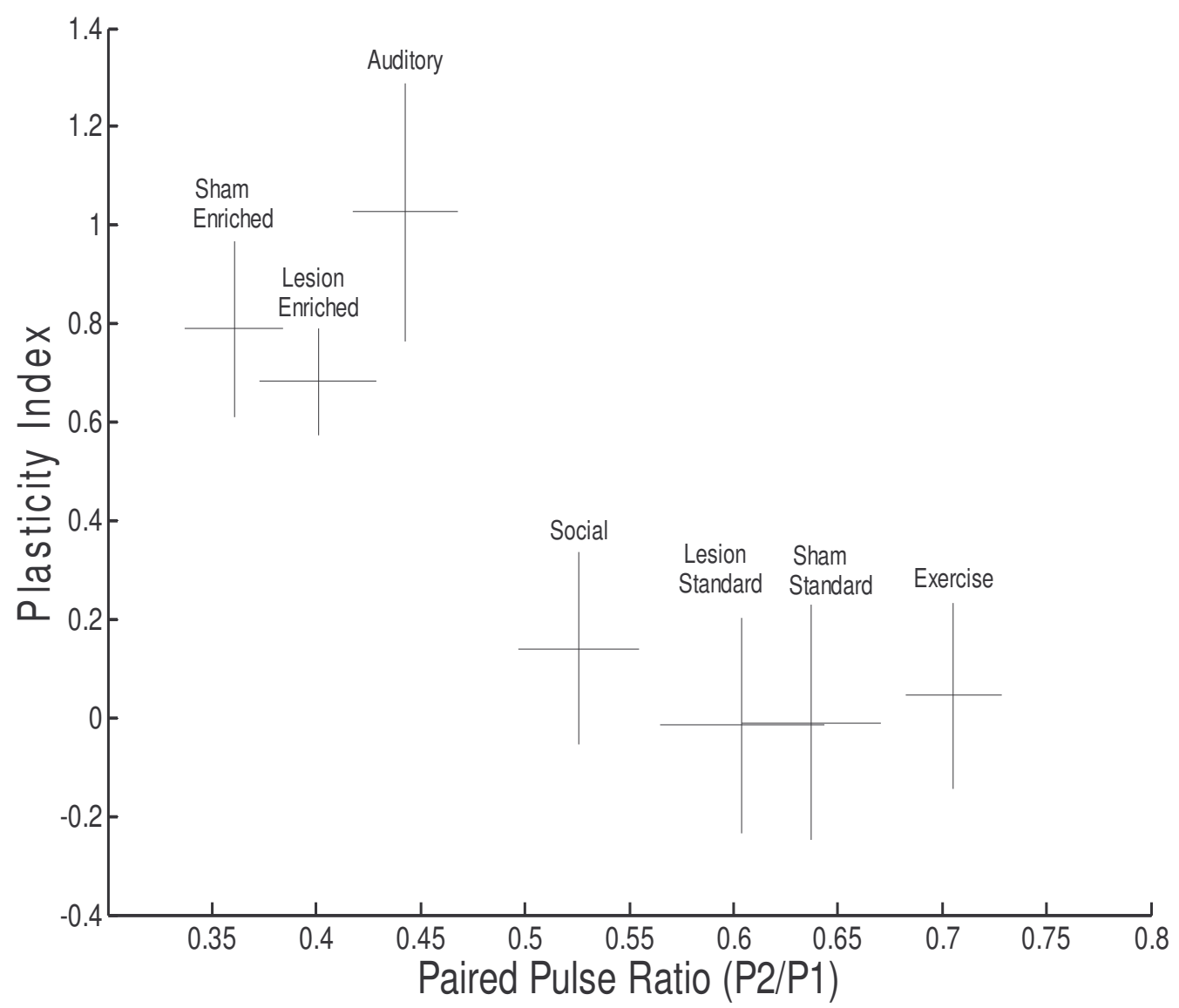

Figure 5.9. Mean plasticity index of each group as a function of the mean paired-pulse ratio. Rats in the sham-enriched, lesion-enriched, and auditory exposure conditions with large response magnitude plasticity had more paired-pulse depression than groups with less response magnitude plasticity. 
Tones vs. Noise Bursts

To determine how environmental plasticity differs when measured with broadband sounds, we recorded evoked potentials in response to three different noise bursts (loud: 70 dB SPL with 3 ms rise and fall times; quiet: $50 \mathrm{~dB}$ SPL with $3 \mathrm{~ms}$ rise and fall times; ramped: $70 \mathrm{~dB}$ SPL with $50 \mathrm{~ms}$ rise and fall times). Averaged across all rats, the $70 \mathrm{~dB}$ loud noise evoked a significantly larger response than the $50 \mathrm{~dB}$ noise $(109 \pm 5 \mathrm{mV}$ vs. $93 \pm 5 \mathrm{mV}$, $\mathrm{p}<0.001)$ and the $50 \mathrm{~dB}$ noise evoked a significantly larger response than the $70 \mathrm{~dB}$ tone (93 $\pm 5 \mathrm{mV}$ vs. $48 \pm 3 \mathrm{mV}, \mathrm{p}<0.001)$. The evoked response to the $70 \mathrm{~dB}$ ramped noise was intermediate between the other two noise bursts $(94 \pm 5 \mathrm{mV}, \mathrm{p}<0.01)$. The effects of auditory enrichment were greatest when measured with tones (Table 5.1). Responses to all three noise bursts were significantly enhanced when rats were housed in the enriched environment, but not during exposure to enrichment sounds alone (Table 5.1). The observation that the auditory exposure group did not show significant changes in response to noise bursts and had smaller changes in PPD suggests that housing within the enriched condition (where rats can trigger sounds and use auditory information to make judgments) results in more plasticity than sensory exposure alone. 


\section{DISCUSSION}

Our previous studies demonstrated that housing rats in an enriched environment increases the cortical response to tone and noise burst stimuli, and increases PPD to repeated tones (Engineer et al., 2004; Percaccio et al., 2005). This study was designed to evaluate the contribution of exercise, social stimulation, and auditory exposure to enrichment-induced plasticity. To investigate the contribution of ACh to enrichment-induced plasticity, evoked potentials were recorded from rats with cholinergic lesions before, during, and after environmental enrichment. The current study confirms that enrichment substantially increases response strength and PPD, identifies auditory sensory experience as the critical determining factor, and demonstrates that unilateral cholinergic damage does not prevent enrichment-induced plasticity in AC.

Both social stimulation and exercise result in anatomical, neurochemical, physiological, and behavioral changes that are similar to those caused by complete environmental enrichment (van Praag et al., 1999; Champagne and Curley, 2005). If the anatomical and neurochemical plasticity that results from enrichment contributes to the physiological changes we have documented in $\mathrm{AC}$, then exercise and social stimulation would increase responses in AC. Our observation that neither exercise nor social interactions are sufficient to increase AC responses to tones or noise bursts suggests that the plasticity induced by physical activity and social housing occur primarily in brain regions activated by these conditions. For example, exercise and social interactions stimulate anatomical plasticity in motor cortex and amygdala, respectively (Anderson et al., 2002; Gordon et al., 
2003). The fact that passive exposure to sounds increases physiologic responses in $\mathrm{AC}$ suggests the possibility that associated anatomical changes are restricted to AC. Additional studies are needed to evaluate this hypothesis.

Although our results indicate that sensory exposure stimulates plasticity in AC, several earlier studies have shown that passive auditory exposure does not increase response strength or temporal processing. Habituation, for example, results in a long-lasting frequency specific decrease in cortical responses to repeated tone presentations (Condon and Weinberger, 1991). Exposing monkeys to meaningless tones while they were engaged in a tactile discrimination task does not alter the organization of the A1 frequency map (Recanzone et al., 1993). Likewise, exposure to noise burst trains does not alter response strength or latency of AC neurons (Bao et al., 2003). The most likely explanation for exposure-induced plasticity is that the enriched environment was sufficiently interesting to cause the rats to actively attend to the events associated with it even though they could not directly participate.

The enriched environment in our studies was designed to be sufficiently complex so that the sounds generated by it would be neither predictable (i.e. boring) nor random (i.e. meaningless). Exposure to the enriched environment may have commanded attention and generated plasticity because the interactions between the rats and the sound sources were optimally unpredictable and interesting to the rats observing the environment. In addition, the social interactions in the enrichment cage, including vocalizations, are situationally dependent, and likely an interesting component of acoustic enrichment. Vocalizations, in and 
of themselves, however, are not sufficient to induce plasticity, given that exercise and standard-housed rats also heard vocalizations from other singly housed rats in the same room. Furthermore, being housed in social isolation with an exercise wheel actually reverses the increase in neurogenesis previously attributed to wheel running (Stranahan et al., 2006). The lack of anatomical plasticity may also explain our negative physiological result in running rats. These results indicate that to generate plasticity environments should contain rich, diverse, and interesting stimuli in addition to vocalizations. For example, rats group housed in a large cage with added objects have greater brain weights than rats individually housed in the enriched cage or rats socially housed in a standard cage (Rosenzweig et al., 1978). Our observation that auditory exposure without social interactions and group housing without additional auditory stimulation lead to intermediate levels of plasticity (Figure 5.8, Table 5.1) are consistent with previous reports that the combination of a complex environment and social stimulation results in the greatest plasticity.

In this study, the plasticity evoked by different housing conditions appears to exist on a continuum. On one end of the continuum, rats that simply heard the enriched environment exhibited less paired-pulse depression and no noise-evoked plasticity compared to rats housed in the enriched environment (Tables $5.1 \& 5.2$ ). Rats in the exposure group may have been unmotivated to attend to sounds since they had less behavioral relevance. On the other end of the continuum, group housed rats exhibit more paired-pulse depression than rats housed with an exercise wheel, possibly because group housed rats were motivated to attend to the vocalizations of their cage mates since they had social consequences. These results 
suggest that effects of environmental enrichment may be manifest as a function of the behavioral importance of sounds in each housing condition. The neural basis for these interactions is likely to be quite complicated, potentially including modulation of neurotransmitter release, stress hormone levels, and arousal.

Modulatory neurotransmitters, including $\mathrm{ACh}$, dopamine, and norepinephrine, are released by arousal and attention and stimulate many forms of plasticity both independently and in concert (Hasselmo, 1995; Gu, 2002). While several forms of cortical plasticity are blocked by removing cholinergic inputs, other forms require removal of more than one neuromodulator (Bear and Singer, 1986; Baskerville et al., 1997; Conner et al., 2003; Kamke et al., 2005). For example, the reorganization that follows digit amputation or nerve transection is blocked by excitotoxic NB lesions that destroy both cholinergic and GABAergic inputs to the cortex (Juliano et al., 1991; Webster et al., 1991). Ocular dominance plasticity can also only be prevented if cholinergic damage is combined with concurrent norepinephrine depletion (Bear and Singer, 1986). Targeted destruction of cholinergic NB neurons that project to cortex does not impair cortical reorganization following cochlear damage, indicating that another neurotransmitter may co-mediate plasticity in AC (Kamke et al., 2005). These observations suggest that the additional destruction of GABAergic innervation from NB or noradrenergic inputs from locus coeruleus may be required to block the cortical effects of environmental enrichment. Future studies that combine lesion protocols are needed to distinguish the relative contribution of each neurotransmitter to environmental plasticity. 


\section{Technical Considerations}

Although considerable evidence suggests that multiple neurotransmitters regulate plasticity, it is possible that the cholinergic lesion technique used in this study failed to block enrichment-induced plasticity because spared cholinergic fibers were sufficient to stimulate plasticity. Over time, there may be substantial cholinergic marker recovery after unilateral injection (Waite et al., 1995; Casamenti et al., 1988). Although it is possible that bilateral cholinergic lesions would interfere with enrichment-induced plasticity, this seems unlikely since the NB projection to the cortex is homolateral, and unilateral ICV injection of 192 IgGsaporin $(6 \mu \mathrm{g})$ is sufficient to block whisker pairing plasticity in somatosensory cortex (Wenk et al., 1980; Baskerville et al., 1997).

We only injected $2.5 \mu \mathrm{g}$ because higher doses damage Purkinje cells and do not result in a significantly greater reduction of cholinergic innervation (Waite et al., 1995; Walsh et al., 1995; Waite and Chen, 2001). In addition, $2.5 \mu \mathrm{g}$ was sufficient to prevent AC map plasticity after NB stimulation- tone pairing (Kilgard and Merzenich, 1998a). Since blocking map plasticity in motor cortex impairs experience-dependent plasticity, it is reasonable to expect enrichment-induced plasticity in the auditory cortex to also be impaired (Conner et al., 2003). However, the normal development of enrichment-induced plasticity in auditory cortex suggests either that acetylcholine is not involved in this form of learning, or that there were effective compensatory mechanisms, including upregulation of intrinsic or surviving cholinergic neurons and/or the involvement of other neuromodulatory systems. Although we are unable to determine the degree of compensation in this experiment, significant $\mathrm{AChE}$ 
damage was still evident more than 20 weeks after the injection. It remains a possibility, however, that a more complete block of cholinergic projections, achieved with either higher doses of toxin or with bilateral lesions, might prevent enrichment induced plasticity.

\section{Clinical Implications}

Sensory gating deficits are associated with several clinical populations, including schizophrenia, autism, and dyslexia. Schizophrenic individuals exhibit weaker responses and less PPD compared to normal controls, and benefit from antipsychotic medications that restore normal PPD (Erwin et al., 1991; Adler et al., 2004). Individuals with autism also exhibit reduced PPD, which might contribute to their propensity for sensory overload (Buchwald et al., 1992; O'Neill and Jones, 1997). Dyslexic individuals often have too much PPD (especially at short interstimulus intervals), consistent with observations of difficulty processing rapid spectrotemporal transitions (Nagarajan et al., 1999). Focal training with complex sounds can alter cortical responses and improve the language processing capabilities of children with autism or dyslexia (Merzenich et al., 1996; Tallal et al., 1996; Merzenich et al., 1999; Hayes et al., 2003). Our results confirm that PPD can be increased or decreased as a function of experience, and suggest that sensory therapies might also be developed for patients with other disorders characterized by sensory processing abnormalities.

Several disorders with sensory gating deficits also have cholinergic abnormalities, including patients with autism, schizophrenia, and Alzheimer's disease (Cullum et al., 1993; Freedman et al., 1994; Jessen et al., 2001; Perry et al., 2001; Gil-Bea et al., 2005; Perry et al., 2006). It has been suggested that cholinergic dysfunction alters PPD (Adler et al., 1998). 
However, our data suggest that sensory gating deficits are not caused by cholinergic lesions and cast doubt on the hypothesis that both the sensory gating deficits and the cognitive dysfunction associated with schizophrenia, autism, and Alzheimer's disease arise from a single cholinergic deficit. For instance, abnormal activity of dopamine also results in sensory gating deficits (Braff and Geyer, 1990). Although the perceptual consequences of environmental enrichment are not well documented, our observations that environment can significantly influence PPD and that enrichment-induced plasticity may be independent of cholinergic function suggests that focused, intensive sensory enrichment may alter sensory gating in clinical populations, despite persistent cholinergic abnormalities.

\section{Ceiling Effect}

While physiological plasticity studies in humans and animals often probe neural responses with intense stimuli, more reliable plasticity may be observed using stimuli that do not elicit saturated responses (Wible et al., 2002). In the present study, the greatest proportional change in evoked response was recorded using sounds that stimulate less than the maximal neural response (Table 5.1). Despite the better signal to noise ratio in recordings evoked by loud noise bursts, environmental plasticity was more reliable and proportionally larger when assayed using tones. Stimuli that evoke smaller neural responses may avoid a potential ceiling effect and reveal a greater influence of enrichment on cortical responsiveness. 


\section{Conclusions}

These experiments confirm that enrichment enhances response strength and PPD in auditory cortex, highlights the importance of sensory experience, and demonstrates that cholinergic damage does not prevent enrichment-induced plasticity. Neither social interactions nor running on a wheel stimulated significant plasticity compared to enrichment. Experiencing the enriched environment from a distance increased paired-pulse depression and response to tones, although noise-evoked responses and temporal plasticity were somewhat diminished compared to rats housed in the enrichment cage. Overall, these results suggest that sensory stimulation has the potential to generate plasticity that may be beneficial for several clinical disorders, including dyslexia, autism, schizophrenia, and Alzheimer's disease. 


\section{REFERENCES}

Adler, L.E., Olincy, A., Cawthra, E.M., McRae, K.A., Harris, J.G., Nagamoto, H.T., Waldo, M.C., Hall, M.H., Bowles, A., Woodward, L., Ross, R.G. and Freedman, R. (2004). Varied effects of atypical neuroleptics on P50 auditory gating in schizophrenia patients. Am J Psychiatry 161:1822-1828.

Adler, L.E., Olincy, A., Waldo, M., Harris, J.G., Griffith, J., Stevens, K., Flach, K., Nagamoto, H., Bickford, P., Leonard, S. and Freedman, R. (1998). Schizophrenia, sensory gating, and nicotinic receptors. Schizophr Bull 24:189-202.

Anderson, B.J., Eckburg, P.B. and Relucio, K.I. (2002). Alterations in thickness of motor cortical subregions after motor skill learning and exercise. Learn Mem 9:1-9.

Bao, S., Chang, E.F., Davis, J.D., Gobeske, K.T. and Merzenich, M.M. (2003). Progressive degradation and subsequent refinement of acoustic representations in the adult auditory cortex. J Neurosci 23:10765-10775.

Baskerville, K.A., Schweitzer, J.B. and Herron, P. (1997). Effects of cholinergic depletion on experience-dependent plasticity in the cortex of the rat. Neuroscience 80:1159-1169.

Bear, M.F. and Singer, W. (1986). Modulation of visual cortical plasticity by acetylcholine and noradrenaline. Nature 320:172-176.

Beaulieu, C. and Cynader, M.. (1990a). Effect of the richness of the environment on neurons in cat visual cortex. I. Receptive field properties. Brain Res Dev Brain Res 53:71-81.

Beaulieu, C. and Cynader, M. (1990b). Effect of the richness of the environment on neurons in cat visual cortex. II. Spatial and temporal frequency characteristics. Dev Brain Res 53:82-88.

Bennett, E.L., Rosenzweig, M.R. and Diamond, M.C. (1969). Rat brain: effects of environmental enrichment on wet and dry weights. Science 163:825-826. 
Bennett, E.L., Krech, D. and Rosenzweig, M.R. (1964). Reliability and regional specificity of cerebral effects of environmental complexity and training. J Comp Physiol Psychol 57:440-441.

Berger-Sweeney, J., Stearns, N.A., Frick, K.M., Beard, B. and Baxter, M.G. (2000). Cholinergic basal forebrain is critical for social transmission of food preferences. Hippocampus 10:729-738.

Black, J.E., Isaacs, K.R., Anderson, B.J., Alcantara, A.A. and Greenough, W.T. (1990). Learning causes synaptogenesis, whereas motor activity causes angiogenesis, in cerebellar cortex of adult rats. Proc Natl Acad Sci USA 87:5568-5572.

Book, A.A., Wiley, R.G. and Schweitzer, J.B. (1992). Specificity of 192 IgG-saporin for NGF receptor-positive cholinergic basal forebrain neurons in the rat. Brain Research 590:350-355.

Braff, D.L. and Geyer, M.A. (1990). Sensorimotor gating and schizophrenia. Human and animal model studies. Arch Gen Psychiatry 47:181-188.

Buchwald, J.S., Erwin, R., Van Lancker, D., Guthrie, D., Schwafel, J. and Tanguay, P. (1992). Midlatency auditory evoked responses: P1 abnormalities in adult autistic subjects. Electroencephalogr Clin Neurophysiol 84:164-171.

Casamenti, F., Di Patre, P.L., Bartolini, L. and Pepeu, G. (1988). Unilateral and bilateral nucleus basalis lesions: differences in neurochemical and behavioral recovery. Neuroscience 24:209-215.

Champagne, F.A. and Curley, J.P. (2005). How social experiences influence the brain. Curr Opin Neuobiol 15:704-709.

Condon, C.D. and Weinberger, N.M. (1991). Habituation produces frequency-specific plasticity of receptive fields in the auditory cortex. Behav Neurosci 105:416-430.

Conner, J.M., Culberson, A., Packowski, C., Chiba, A.A. and Tuszynski, M.H. (2003). Lesions of the basal forebrain cholinergic system impair task acquisition and abolish cortical plasticity associated with motor skill learning. Neuron 38:819-829. 
Coq, J.O. and Xerri, C. (1998). Environmental enrichment alters organizational features of the forepaw representation in the primary somatosensory cortex of adult rats. Exp Brain Res 121:191-204.

Cullum, C.M., Harris, J.G., Waldo, M.C., Smernoff, E., Madison, A., Nagamoto, H.T., Griffith, J.M., Adler, L.E. and Freedman, R. (1993). Neurophysiological and neuropsychological evidence for attentional dysfunction in schizophrenia. Schizophr Res 10:131-141.

de Castro, J.M. and Duncan, G. (1985). Operantly conditioned running: effects on brain catecholamine concentrations and receptor densities in the rat. Pharmacol Biochem Behav 23:495-500.

Delacour, J., Houcine, O. and Costa, J.C. (1990). Evidence for a cholinergic mechanism of "learned" changes in the responses of barrel field neurons of the awake and undrugged rat. Neuroscience 34:1-8.

Diamond, M.C. (2001). Response of the brain to enrichment. An Acad Bras Cienc 73:211220.

Diamond, M.C., Law, F., Rhodes, H., Lindner, B., Rosenzweig, M.R., Krech, D. and Bennett, E.L. (1966). Increases in cortical depth and glia numbers in rats subjected to enriched environment. J Comp Neurol 128:117-126.

Einon, D.F., Humphreys, A.P., Chivers, S.M., Field, S. and Naylor, V. (1981). Isolation has permanent effects upon the behavior of the rat, but not the mouse, gerbil, or guinea pig. Dev Psychobiol 14:343-355.

Engineer, N.D., Percaccio, C.R., Pandya, P.K., Moucha, R., Rathbun, D.L. and Kilgard, M.P. (2004). Environmental enrichment improves response strength, threshold, selectivity, and latency of auditory cortex neurons. J Neurophysiol 92:73-82.

Erwin, R.J., Mawhinney-Hee, M., Gur, R.C. and Gur, R.E. (1991). Midlatency auditory evoked responses in schizophrenia. Biol Psychiatry 30:430-442. 
Farmer, J., Zhao, X., van Praag, H., Wodtke, K., Gage, F.H. and Christie, B.R. (2004). Effects of voluntary exercise on synaptic plasticity and gene expression in the dentate gyrus of adult male Sprague-Dawley rats in vivo. Neuroscience 124:71-79.

Ferchmin, P.A. and Bennett, E.L. (1975). Direct contact with enriched environment is required to alter cerebral weights in rats. J Comp Physiol Psychol 88:360-367.

Ferriera, G., Meurisse, M., Gervais, R., Ravel, I.V. and Levy, F. (2001). Extensive immunolesions of basal forebrain cholinergic system impair offspring recognition in sheep. Neuroscience 106:103-116.

Freedman, R., Adler, L.E., Bickford, P., Byerley, W., Coon, H., Cullum, C.M., Griffith, J.M., Harris, J.G., Leonard, S. and Miller, C. (1994). Schizophrenia and nicotinic receptors. Harv Rev Psychiatry 2:179-192.

Galani, R., Jeltsch, H., Lehmann, O., Bertrand, F. and Cassel, J.C. (2002). Effects of 192 IgG-saporin on acetylcholinesterase histochemistry in male and female rats. Brain Res Bul 58:179-186.

Geyer, M.A., Wilkinson, L.S., Humby, T. and Robbins, T.W. (1993). Isolation rearing of rats produces a deficit in prepulse inhibition of acoustic startle similar to that in schizophrenia. Biol Psychiatry 34:361-372.

Gil-Bea, E.J., Garcia-Alloza, M., Dominguez, J., Marcos, B. and Ramirez, M.J. (2005). Evaluation of cholinergic markers in Alzheimer's disease and in a model of cholinergic deficit. Neurosci Lett 375:37-41.

Gordon, N.S., Burke, S., Akil, H., Watson, S.J. and Panksepp, J. (2003). Socially-induced brain 'fertilization': play promotes brain derived neurotrophic factor transcription in the amygdala and dorsolateral frontal cortex in juvenile rats. Neurosci Lett 341:17-20.

Greenough, W.T., Volkmar, F.R. and Juraska, J.M. (1973). Effects of rearing complexity on dendritic branching in frontolateral and temporal cortex of the rat. Exp Neurol 41:371-378. 
$\mathrm{Gu}$, Q. (2002). Neuromodulatory transmitter systems in the cortex and their role in cortical plasticity. Neuroscience 111:815-835.

Gu, Q. and Singer, W. (1993). Effects of intracortical infusion of anticholinergic drugs on neuronal plasticity in kitten striate cortex. Eur J Neurosci 5:475-485.

Hasselmo, M.E. (1995). Neuromodulation and cortical function: modeling the physiological basis of behavior. Behav Brain Res 67:1-27.

Hayes, E.A., Warrier, C.M., Nicol, T.G., Zecker, S.G. and Kraus, N. (2003). Neural plasticity following auditory training in children with learning problems. Clin Neurophysiol 114:673-684.

Heckers, S., Ohtake, T., Wiley, R.G., Lappi, D.A., Geula, C. and Mesulam, M.M. (1994). Complete and selective cholinergic denervation of rat neocortex and hippocampus but not amygdala by an immunotoxin against the p75 NGF receptor. $J$ Neurosci 14:12711289.

Ickes, B.R., Pham, T.M., Sanders, L.A., Albeck, D.S., Mohammed, A.H. and Granholm, A.C. (2000). Long-term environmental enrichment leads to regional increases in neurotrophin levels in rat brain. Exp Neurol 164:45-52.

Ivliev, D.A. (1999). The effects of atropine microinjections into the motor cortex of rats on the development of a motor habit. Neurosci Behav Physiol 29:371-375.

Jessen, F., Kucharski, C., Fries, T., Papassotiropoulos, A., Hoenig, K., Maier, W. and Heun, R. (2001). Sensory gating deficit expressed by a disturbed suppression of the P50 event-related potential in patients with Alzheimer's disease. Am J Psychiatry 158:1319-1321.

Johansson, B.B. (2003). Environmental influence on recovery after brain lesions-experimental and clinical data. J Rehabil Med 41:11-16.

Juliano, S.L., Ma, W. and Eslin, D. (1991). Cholinergic depletion prevents expansion of topographic maps in somatosensory cortex. Proc Natl Acad Sci USA 88:780-784. 
Juliano, S.L., Ma, W., Bear, M.F. and Eslin, D. (1990). Cholinergic manipulation alters stimulus-evoked metabolic activity in cat somatosensory cortex. J Comp Neurol 297:106-120.

Kamke, M.R., Brown, M. and Irvine, D.R. (2005). Origin and immunolesioning of cholinergic basal forebrain innervation of cat primary auditory cortex. Hear Res 206:89-106.

Karnovsky, M.J. and Roots, L. (1964). A "direct-coloring" thiocholine method for cholinesterases. J Histochem Cytochem 12:219-221.

Katz, H.B. and Davies, C.A. (1984). Effects of differential environments on the cerebral anatomy of rats as a function of previous and subsequent housing conditions. Exp Neurol 83:274-287.

Kilgard, M.P. and Merzenich, M.M. (1998a). Cortical map reorganization enabled by nucleus basalis activity. Science 279:1714-1718.

Kilgard, M.P. and Merzenich, M.M. (1998b). Plasticity of temporal information processing in the primary auditory cortex. Nat Neurosci 1:727-731.

Kiss, J., McGovern, J. and Patel, A.J. (1988). Immunohistochemical localization of cells containing nerve growth factor receptors in the different regions of the adult rat forebrain. Neuroscience 27:731-48.

Kudoh, M., Seki, K. and Shibuki, K. (2004). Sound sequence discrimination learning is dependent on cholinergic inputs to the rat auditory cortex. Neurosci Res 50:113-123.

Lewis, E.M., Barnett, J.F., Jr., Freshwater, L., Hoberman, A.M. and Christian, M.S. (2002). Sexual maturation data for Crl Sprague-Dawley rats: criteria and confounding factors. Drug Chem Toxicol 25:437-458.

Mayo, W., Kharouby, M., Le Moal, M. and Simon, H. (1988). Memory disturbances following ibotenic acid injections in nucleus basalis magnocellularis of the rat. Brain Res 455:213-222. 
Merzenich, M.M., Jenkins, W.M., Johnston, P., Schreiner, C., Miller, S.L. and Tallal, P. (1996). Temporal processing deficits of language-learning impaired children ameliorated by training. Science 271:77-81.

Merzenich, M.M., Sauders, G., Jenkins, W.M., Miller, S., Peterson, B. and Tallal, P. (1999). Pervasive developmental disorders: Listening training and language abilities. In The changing nervous system: Neurobehavioral consequences of early brain disorders, ed. SH Broman and JM Fletcher, 365-88. New York: Oxford University Press.

Metherate, R., Tremblay, N. and Dykes, R.W. (1987). Acetylcholine permits long-term enhancement of neuronal responsiveness in cat primary somatosensory cortex. Neurosci 22:75-81.

Metherate, R. and Weinberger, N.M. (1989). Acetylcholine produces stimulus-specific receptive field alterations in cat auditory cortex. Brain Res 480:372-377.

Motooka, Y., Kondoh, T., Nomura, T., Tamaki, N., Tozaki, H., Kanno, T. and Nishizaki, T. (2001). Selective cholinergic denervation inhibits expression of long-term potentiation in the adult but not infant rat hippocampus. Dev Brain Res 129:119-123.

Nagarajan, S.S., Mahncke, H., Salz, T., Tallal, P., Roberts, T., and Merzenich, M.M. (1999). Cortical auditory signal processing in poor readers. Proc Natl Acad Sci U S A 96:6483-6488.

Naka, F., Shiga, T., Yaguchi, M. and Okado, N. (2002). An enriched environment increases noradrenaline concentration in the mouse brain. Brain Res 924:124-126.

Neeper, S.A., Gomez-Pinilla, F., Choi, J. and Cotman, C.W. (1996). Physical activity increases mRNA for brain-derived neurotrophic factor and nerve growth factor in rat brain. Brain Res 726:49-56.

Nilsson, O.G., Leanza, G., Rosenblad, C., Lappi, D.A., Wiley, R.G. and Bjorklund, A. (1992). Spatial learning impairments in rats with selective immunolesions of the forebrain cholinergic system. Neuroreport 3:1005-1008. 
O'Neill, M. and Jones, R.S. (1997). Sensory-perceptual abnormalities in autism: a case for more research? J Autism Dev Disord 27:283-293.

O'Shea, L., Saari, M., Pappas, B.A., Ings, R. and Stange, K. (1983). Neonatal 6hydroxydopamine attenuates the neural and behavioral effects of enriched rearing in the rat. Eur J Pharmacol 92:43-47.

Paban, V., Chambon, C., Jaffard, M. and Alescio-Lautier, B. (2005). Behavioral effects of basal forebrain cholinergic lesions in young adult and aging rats. Behav Neurosci 119:933-945.

Park, G.A., Pappas, B.A., Murtha, S.M. and Ally, A. (1992). Enriched environment primes forebrain choline acetyltransferase activity to respond to learning experience. Neurosci Lett 143:259-262.

Paxinos, G. and Watson, C. (1998). The Rat Brain in Stereotaxic Coordinates. San Diego, CA:Academic Press.

Percaccio, C.R., Engineer, N.D., Pruette, A.L., Pandya, P.K., Moucha, R., Rathbun, D.L. and Kilgard, M.P. (2005). Environmental enrichment increases paired-pulse depression in rat auditory cortex. J Neurophysiol 94:3590-3600.

Perry, E.K., Lee, M.L., Martin-Ruiz, C.M., Court, J.A., Volsen, S.G., Merrit, J., Folly, E., Iversen, P.E., Bauman, M.L., Perry, R.H. and Wenk, G.L. (2001). Cholinergic activity in autism: abnormalities in the cerebral cortex and basal forebrain. Am J Psychiatry 158:1058-1066.

Perry, W., Minassian, A., Lopez, B., Maron, L. and Lincoln, A. (2006). Sensorimotor gating deficits in adults with autism. Biol Psychiatry [E pub ahead of print].

Por, S.B., Bennett, E.L. and Bondy, S.C. (1982). Environmental enrichment and neurotransmitter receptors. Behav Neural Biol 34:132-140. 
Preece, M.A., Dalley, J.W., Theobald, D.E., Robbins, T.W. and Reynolds, G.P. (2004). Region specific changes in forebrain 5-hydroxytryptamine1A and 5hydroxytryptamine $2 \mathrm{~A}$ receptors in isolation-reared rats: an in vitro autoradiography study. Neuroscience 123:725-732.

Recanzone, G.H., Schreiner, C.E. and Merzenich, M.M. (1993). Plasticity in the frequency representation of primary auditory cortex following discrimination training in adult owl monkeys. J Neurosci 13:87-103.

Richardson, R.T. and DeLong, M.R. (1991). Electrophysiological studies of the functions of the nucleus basalis in primates. Adv Exp Med Biol 295:233-252.

Risedal, A., Mattsson, B., Dahlqvist, P., Nordborg, C., Olsson, T. and Johansson, B.B. (2002). Environmental influences on functional outcome after a cortical infarct in the rat. Brain Res Bull 58:315-321.

Rosenzweig, M.R., Bennett, E.L., Hebert, M. and Morimoto, H. (1978). Social grouping cannot account for cerebral effects of enriched environments. Brain Res 153:563-576.

Sato, H., Hata, Y., Hagiharak, K. and Tsumoto, T. (1987). Effect of cholinergic depletion on neuron activity in the cat visual cortex. J Neurophysiol 58:781-794.

Stummer, W., Weber, K., Tranmer, B., Baethmann, A. and Kempski, O. (1994). Reduced mortality and brain damage after locomotor activity in gerbil forebrain ischemia. Stroke 25:1862-1869.

Swain, R.A., Harris, A.B., Wiener, E.C., Dutka, M.V., Morris, H.D., Theien, B.E., Konda, S., Engberg, K., Lauterbur, P.C. and Greenough, W.T. (2003). Prolonged exercise induces angiogenesis and increases cerebral blood volume in primary motor cortex of the rat. Neuroscience 117:1037-1046.

Tallal, P., Miller, S.L., Bedi, G., Byma, G., Wang, X., Nagarajan, S.S., Schreiner, C., Jenkins, W.M. and Merzenich, M.M. (1996). Language comprehension in languagelearning impaired children improved with acoustically modified speech. Science 271:81-84. 
Turner, A.M. and Greenough, W.T. (1985). Differential rearing effects on rat visual cortex synapses. I. Synaptic and neuronal density and synapses per neuron. Brain Res 329:195-203.

van Praag, H., Kempermann, G. and Gage, F.H. (2000). Neural consequences of environmental enrichment. Nat Rev Neurosci 1:191-198.

van Praag, H., Kempermann, G. and Gage, F.H. (1999). Running increases cell proliferation and neurogenesis in the adult mouse dentate gyrus. Nat Neurosci 2:266-270.

Verdier, D. and Dykes, R.W. (2001). Long-term cholinergic enhancement of evoked potentials in rat hindlimb somatosensory cortex displays characteristics of long-term potentiation. Exp Brain Res 137:71-82.

Waite, J.J. and Chen, A.D. (2001). Differential changes in rat cholinergic parameters subsequent to immunotoxic lesion of the basal forebrain nuclei. Brain Res 918:113120.

Waite, J.J., Chen, A.D., Wardlow, M.L., Wiley, R.G., Lappi, D.A. and Thal, L.J. (1995). 192 immunoglobulin G-saporin produces graded behavioral and biochemical changes accompanying the loss of cholinergic neurons of the basal forebrain and cerebellar purkinje cells. Neuroscience 65:463-476.

Waite, J.J., Wardlow, M.L., Chen, A.C., Lappi, D.A., Wiley, R.G. and Thal, L.J. (1994). Time-course of cholinergic and monoaminergic changes in rat brain after immunolesioning with 192 IgG-saporin. Neuroscience Letters 169:154-158.

Walsh T.J., Kelly R.M., Dougherty K.D., Stackman R.W., Wiley R.G. and Kutscher C.L. (1995). Behavioral and neurobiological alterations induced by the immunotoxin 192IgG-saporin:cholinergic and non-cholinergic effects following i.c.v. injection. Brain Res 702:233-245.

Webster H.H., Hanisch U.K., Dykes R.W. and Biesold D. (1991). Basal forebrain lesions with or without reserpine injection inhibit cortical reorganization in rat hindpaw primary somatosensory cortex following sciatic nerve section. Somatosens Mot Res 8: 327-346. 
Wenk H., Bigl V. and Meyer U. (1980). Cholinergic projections from magnocellular nuclei of the basal forebrain to cortical areas in rats. Brain Res 2: 295-316.

Wible B., Nicol, T. and Kraus, N. (2002). Abnormal neural encoding of repeated speech stimuli in noise in children with learning problems. Clinical Neurophysiology 113(4): 459-61.

Wiley R.G., Berbos T.G., Deckwerth T.L., Johnson E.M., Jr. and Lappi D.A. (1995). Destruction of the cholinergic basal forebrain using immunotoxin to rat NGF receptor: modeling the cholinergic degeneration of Alzheimer's disease. J Neurol Sci 128: $157-166$.

Wiley R.G., Oeltmann T.N. and Lappi D.A. (1991). Immunolesioning:selective destruction of neurons using immunotoxin to rat NGF receptor. Brain Res 562: 149-153. 


\section{CHAPTER 6}

\section{CONCLUSION}

Low-functioning children with autism are stuck in the sensory stage of play. This is the most basic form of play and is characterized by a need to explore objects with their eyes, hands, and mouths. If a child still has gaps in their understanding of the sensory nature of the world, they will be impaired in their ability to understand how (or why) sensory properties (must continuously) change. Play therapy helps them acquire meaning, progress to higher play stages and cognitive levels, and eventually diminishes autistic symptoms (MacAlpine, 1998). As an early graduate student, I was determined to understand the remarkable selforganizing capacity of the brain that enables this recovery. In a concerted effort to bridge the gap between clinical and basic neuroscience, the experiments contained within my dissertation were designed to investigate 1) the physiological correlates of improved sensory processing, and 2) how neurons assign meaning to sensory events in the environment (both of which probably contribute to plasticity). Concern for the child stuck in sensory play has been with me the entire journey. I hope my studies of the relationship between environment and brain function will have applications in both the clinical and research domains.

For the experiments in chapters 2 and 3,21 rats were housed either in standard laboratory conditions or in an enriched environment and evoked responses were recorded from auditory cortex each week for up to 5 months. In chapter 4 , neurophysiologic responses were recorded from rats housed in environments designed to determine whether exercise, social stimulation, and sensory exposure contributed to the enrichment induced plasticity 
documented in chapters 2 and 3. Evoked potentials were also recorded each week for several months from other rats with cholinergic or sham lesions housed in standard laboratory conditions or an enriched environment. We quantified responses to repeated tones and noise bursts to document changes in response strength and paired-pulse depression.

Over the last 50 years, environmental enrichment has been shown to generate more than a dozen changes in brain anatomy and neurochemistry that develop over several weeks (Bennett et al., 1966; Diamond et al., 1966, 1972; Schapiro et al., 1970; Volkmar et al., 1972; Globus et al., 1973; Greenough et al., 1973; Sirevaag et al., 1985; Park et al., 1992; Naka et al., 2002). Physiological plasticity can develop (and fade) on a considerably shorter time scale. In less than 2 weeks, enrichment dramatically increased the strength of tone and noise-evoked responses, but the effects did not last longer than 1 week after return to the standard environment (Engineer et al., 2004). In every case, the mean plasticity index significantly decreased when rats were moved from the enriched to the standard condition and significantly increased when rats were moved from the standard to the enriched condition. There were no significant differences in the scale of plasticity that occurred in young and adult rats, indicating that auditory cortex neurons are remarkably sensitive to environmental conditions throughout life.

Paired-pulse depression also increased within days of environmental enrichment and was restored to control levels after return to standard housing condition (Percaccio et al., 2005). At every interstimulus interval that was a significant degree of paired-pulse depression regardless of housing condition; however, there was significantly more pairedpulse depression when rats were housed in an enriched environment compared to standard 
housing. These results indicate that enrichment substantially increases the degree of response suppression caused by preceding sounds.

Sensory gating deficits are associated with several clinical populations, including individuals with schizophrenia, autism, and dyslexia. Schizophrenic individuals, for example, exhibit weaker responses and less PPD compared to normal controls, and benefit from antipsychotic medications that restore normal PPD (Siegel et al., 1984; Braff et al., 1990; Erwin et al., 1991;Adler et al., 2004). Individuals with autism also exhibit reduced PPD, which might contribute to their propensity for sensory overload (Buchwald et al., 1992; O'Neill et al., 1997). Dyslexic individuals often have too much PPD (especially at short interstimulus intervals), consistent with observations of difficulty processing rapid spectrotemporal transitions (Nagarajan et al., 1999). Focal training with complex sounds can alter cortical responses and improve the language processing capabilities of children with autism or dyslexia (Recanzone et al., 1992; Merzenich et al., 1996; Tallal et al., 1996; Nagarajan et al., 1998; Merzenich et al., 1999; Tremblay et al., 2001; Beitel et al., 2003; Hayes et al., 2003; Warrier et al., 2004). Our results confirm that PPD can be increased or decreased as a function of experience, and suggest that sensory therapies might also be developed for patients with other disorders characterized by sensory processing abnormalities.

Several disorders with sensory gating deficits also have cholinergic abnormalities, including patients with autism, schizophrenia, and Alzheimer's disease (Jessen et al., 2001; Potter et al., 2006; Perry et al., 2001; Chu et al., 2005; Adams et al., 1998). It has been suggested that cholinergic dysfunction alters PPD (Adler et al., 1998). Cholinergic neurons 
were selectively lesioned with the immunotoxin 192 IgG-saporin, to investigate the contribution of acetylcholine to enrichment-induced plasticity. Enrichment increased response strength and forward masking to the same extent in both sham and nucleus basalis lesioned rats. These results indicate that a significant degree of cholinergic damage $(54 \pm 7 \%)$ does not prevent enrichment-induced plasticity, and that other neurotransmitters may be involved. Our data suggests that sensory gating deficits are not caused by cholinergic lesions and casts doubt on the hypothesis that both the sensory gating and the cognitive dysfunction associated with schizophrenia, autism, and Alzheimer's disease arise from a single cholinergic deficit. Although the perceptual consequences of environmental enrichment are not well documented, our observations that environment can significantly influence PPD and that enrichment-induced plasticity may be independent of cholinergic function supports earlier hypothesis that focused, intensive sensory enrichment may alter sensory gating in clinical populations, despite persistent cholinergic abnormalities.

Multiple factors can influence the degree of environmental-induced plasticity including physical activity, social experience, and behavioral relevance of sensory events (van Praag et al., 2000). To determine what aspects of the enriched environment were responsible for the observed changes in response amplitude and paired-pulse depression, we compared tone-evoked responses for rats housed with an exercise wheel, rats housed in a social environment, and rats that were passively exposed to the auditory component of the enrichment cage to the responses of rats housed in the standard environment. During the period of differential housing, the average tone-evoked potential amplitude of the auditory exposure group increased 2-fold, while the response of rats in the exercise and social groups 
were not altered. Paired-pulse depression was also increased during periods of auditory exposure, but, compared to enrichment, was not significantly altered by exercise or social housing. These results indicate that auditory sensory exposure is the critical factor of the enriched environment that generated the increased amplitude and enhanced paired-pulse depression documented in our previous studies (Engineer et al., 2004; Percaccio et al., 2005).

Our results indicate that environmental plasticity may exist on a spectrum (Figure 6.1). When response strength is plotted as a function of paired-pulse depression (Figure 5.8), at first glance, there are two clusters: 1) housing conditions that increase response magnitude and paired-pulse depression, and (2) housing conditions which neither increase response strength nor paired-pulse depression. However, on closer inspection, although the group that was passively exposed to the enriched environment had increased tone-evoked response strength and paired-pulse depression, there was less paired-pulse depression compared to sham-enriched rats, and no noise-evoked plasticity. Additionally, although rats housed in the social condition did not have increased response strength, they did have significantly more paired-pulse depression than standard-housed rats and rats housed with a running wheel, and were not different from rats that heard the enriched environment. These results indicate that temporal physiological plasticity, at least, may exist on a continuum. This pattern of results may be the consequence of 1) less motivation to attend to sounds in the passive exposure condition, since they are not behaviorally relevant, and 2) more motivation to attend to vocalizations and interactions in the social condition. Vocalizations without social consequences, heard by rats housed in isolation, for example, are not sufficient to induce 
plasticity. In conclusion, the effects of environmental enrichment may be manifest as a function of the interest and diversity of sounds in the current housing condition.

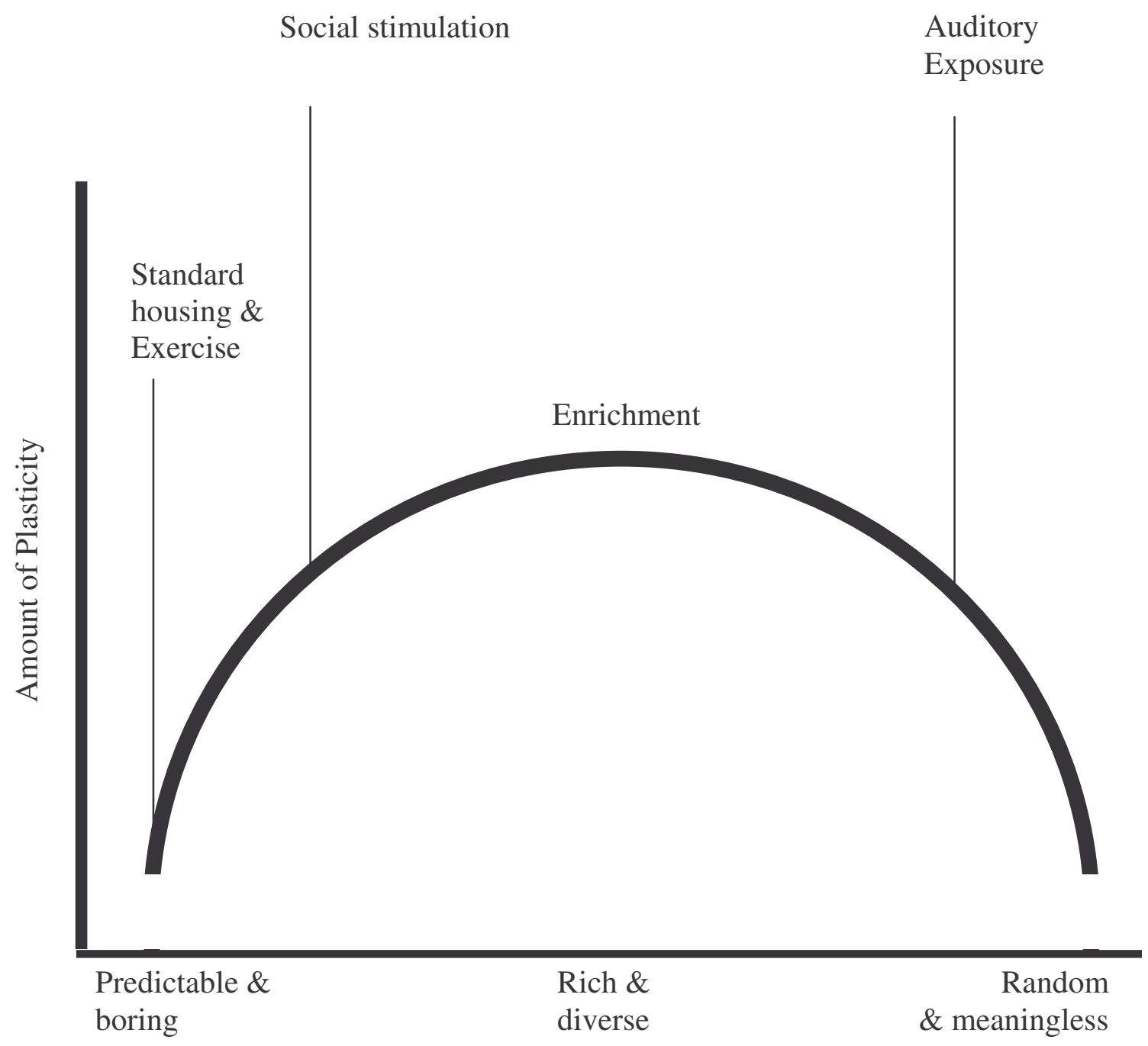

Environment

Figure 6.1. Plasticity along a continuum of environmental interest and diversity. Social stimulation, complete enrichment, and auditory exposure yielded varying digress of temporal plasticity that may have been related to the behavioral relevance of sensory stimuli in the respective environments. 
Although tones evoke a smaller response than noise bursts, environmental plasticity was proportionally larger and more reliable when measured with tones. The larger influence of environment on tone-evoked responses may be due to a ceiling effect caused by the near maximal activation of auditory cortex by noise bursts. To test this hypothesis, we recorded evoked potentials from the same rats in response to three different noise bursts (loud: $70 \mathrm{~dB}$ SPL with 3 ms rise and fall times; quiet: $50 \mathrm{~dB}$ SPL with $3 \mathrm{~ms}$ rise and fall times; ramped: 70 dB SPL with $50 \mathrm{~ms}$ rise and fall times). The $70 \mathrm{~dB}$ "loud" noise-evoked a significantly larger response than the $50 \mathrm{~dB}$ noise $(\mathrm{p}<0.05)$ and the $50 \mathrm{~dB}$ noise-evoked a significantly larger response than the $70 \mathrm{~dB}$ tone $(\mathrm{p}<0.05)$. The evoked response to the $70 \mathrm{~dB}$ ramped noise was intermediate between the other two noise bursts. These results confirm our hypothesis that plasticity is most readily observed with sounds that do not saturate the response of auditory cortex.

In conclusion, future studies will be needed to determine whether behavioral therapies for autism enhance cortical processing of sensory information. Although it is tempting to measure responses to loud sounds and maximize the signal to noise ratio of the evoked potential, my results suggest such an approach may be misguided and could obscure important plasticity. Given the high variability in behavioral measures of autistic traits, the addition of physiological measures may facilitate the evaluation and improvement of current therapies for autism spectrum disorders. 


\section{REFERENCES}

Adams, C.E. and Stevens, K.E. (1998). Inhibition of nitric oxide synthase disrupts inhibitory gating of auditory responses in rat hippocampus. J Pharmacol Exp Ther 287(2): 7605.

Adler, L.E., Olincy, A. Waldo, M., Harris J.G., Griffith J., Stevens K., Flach K., Nagamoto, H., Bickford, P., Lenoard, S. and Freedman, R. (1998). Schizophrenia, sensory gating, and nicotinic receptors. Schizophr Bull 24(2): 189-202.

Adler, L. E., Olincy, A., Cawthra, E.M., McRae, K.A., Harris, J.G., Nagamoto, H.T., Waldo, M.C., Hall, M.H., Bowles, A., Woodward, L., Ross, R.G. and Freedman, R. (2004). Varied effects of atypical neuroleptics on P50 auditory gating in schizophrenia patients. Am J Psychiatry 161(10): 1822-8.

Beitel, R.E., Schreiner, C.E., Cheung, S.W., Wang, X., and Merzenich, M.M. (2003). Reward-dependent plasticity in the primary auditory cortex of adult monkeys trained to discriminate temporally modulated signals. PNAS 100(19): 11070-11075.

Bennett, E.L., Diamond, M.C., Morimoto, H. and Hebert, M. (1966). Acetylcholinesterase activity and weight measures in fifteen brain areas from six lines of rats. $J$ Neurochem, 13: 563-72.

Braff, D.L. and Geyer, M.A. (1990). Sensorimotor gating and schizophrenia. Human and animal model studies. Arch Gen Psychiatry 47(2): 181-8.

Buchwald, J. S., Erwin, R., Van Lancker, D., Guthrie, D., Schwafel, J., and Tanguay, P. (1992). Midlatency auditory evoked responses: P1 abnormalities in adult autistic subjects." Electroencephalogr Clin Neurophysiol. 84: 164-171.

Chu, L.W., Ma, E.S., Lam, K.K., Chan, M.F., Lee, D.H. (2005). Increased alpha 7 nicotinic acetylcholine receptor protein levels in Alzheimer's disease patients. Dement Geriatr Cogn Disord 19(2-3): 106-12.

Diamond, M.C., Law, F., Rhodes, H., Linder, B., Rosenzweig, M.R., Krech, D. and Bennett, E.L. (1966.) Increases in cortical depth and glia numbers in rats subjected to enriched environment. J Comp Neurol 128(1): 117-26.

Diamond, M.C., Rosenzweig, M.R., Bennett, E.L., Linder, B. and Lyon L. (1972.) Effects of environmental enrichment and impoverishment on rat cerebral cortex. J Neurobiol 3(1): 47-64. 
Engineer N.D., Percaccio, C.R., Pandya, P.K., Moucha, R., Rathbun, D.L. and Kilgard, M.P. (2004). Environmental enrichment improves response strength, threshold, selectivity, and latency of auditory cortex neurons. J Neurophysiol 92: 73-82,.

Erwin, R.J., Mawhinney-Hee, M., Gur, R.C. and Gur, R.E. (1991). Midlatency auditory evoked responses in schizophrenia. Biol Psychiatry 30(5): 430-42.

Globus, A., Rosenzweig, M.R., Bennett, E.L. and Diamond, M.C. (1973). Effects of differential experience on dendritic spine counts in rat cerebral cortex. J Comp Physiol Psychol 82(2): 175-81.

Greenough, W.T., Volkmar, F.R. and Juraska, J.M. (1973). Effects of rearing complexity on dendritic branching in frontolateral and temporal cortex of the rat. Exp Neurol, 41: 371-8.

Hayes, E.A., Warrier, C.M., Nicol, T.G., Zecker, S.G. and Kraus, N. (2003). Neural plasticity following auditory training in children with learning problems. Clin Neurophysiol. 114(4): 673-684.

Jessen F., Kucharski, C., Fries, T., Papassotiropoulos, A., Hoenig, K., Maier, W. and Heun, R. (2001.) Sensory gating deficit expressed by a disturbed suppression of the P50 event-related potential in patients with Alzheimer's disease. Am J Psychiatry 158:1319-1321.

MacAlpine, M.L. (1998). Microdevelopment in autism: The power of play. Ph.D. diss., University of Texas at Dallas.

Merzenich, M.M., Jenkins, W.M., Johnston, P., Schreiner, C., Miller, S.L. and Tallal, P. (1996). Temporal processing deficits of language-learning impaired children ameliorated by training. Science 271:77-81.

Merzenich, M.M., Sauders, G., Jenkins, W.M., Miller, S., Peterson, B. and Tallal, P. (1999). Pervasive developmental disorders: Listening training and language abilities. In The changing nervous system: Neurobehavioral consequences of early brain disorders, ed. SH Broman and JM Fletcher, 365-88. New York: Oxford University Press. 
Nagarajan, S.S., Blake, D.T., Wright, B.A., Byl, N. and Merzenich, M.M. (1998). Practicerelated improvements in somatosensory interval discrimination are temporally specific but generalize across skin location, hemisphere, and modality. J Neurosci 18: 1559-1570.

Nagarajan, S.S., Mahncke, H., Salz, T., Tallal, P., Roberts, T., Merzenich, M.M. (1999). Cortical auditory signal processing in poor readers. PNAS 96: 6483-6488.

Naka, F., Shiga, T., Yaguchi, M. and Okado, N. (2002). An enriched environment increases noradrenaline concentration in the mouse brain. Brain Res 924: 124-126.

O'Neill, M. and Jones, R.S. (1997). Sensory-perceptual abnormalities in autism: a case for more research? J Autism Dev Disord 27(3): 283-93.

Park, G.A., Pappas, B.A., Murtha, S.M. and Ally, A. (1992). Enriched environment primes forebrain choline acetyltransferase activity to respond to learning experience. Neurosci Lett 143: 259-262.

Percaccio, C.R., Engineer, N.D., Pruette, A.L., Pandya, P.K., Moucha, R., Rathbun, D.L. and Kilgard, MP. (2005). Environmental enrichment increases paired-pulse depression in rat auditory cortex. J Neurophysiol 94: 3590-3600.

Perry, E.K., Lee, M.L., Martin-Ruiz, C.M., Court, J.A., Volsen, S.G., Merrit, J., Folly, E., Iversen, P.E., Bauman, M.L., Perry, R.H. and Wenk, G.L. (2001). Cholinergic activity in autism: abnormalities in the cerebral cortex and basal forebrain. Am J Psychiatry 158: 1058-1066.

Potter, D., Summerfelt, A., Gold, J. and Buchanan, R.W. (2006). Review of Clinical Correlates of P50 Sensory Gating Abnormalities in Patients with Schizophrenia. Schizophr Bull. [Epub ahead of print].

Recanzone, G.H., Merzenich, M.M. and Schreiner, C.E. (1992) Changes in the distributed temporal response properties of SI cortical neurons reflect improvements in performance on a temporally based tactile discrimination task. J Neurophysiol 67: 1071-1091.

Schapiro, S. and Vukovich, K.R. (1970). Early experience effects upon cortical dendrites: a proposed model for development. Science 167(916): 292-4. 
Siegel, C., Waldo, M., Mizner, G., Adler, L.E. and Freedman, R. (1984). Deficits in sensory gating in schizophrenic patients and their relatives. Arch Gen Psychiatry 41: 607612.

Sirevaag, A.M. and Greenough, W.T. (1985). Differential rearing effects on rat visual cortex synapses. II. Synaptic morphometry. Brain Res 351(2): 215-26.

Tallal, P., Miller, S.L., Bedi, G., Byma, G., Wang, X., Nagarajan, S.S., Schreiner, C., Jenkins, W.M. and Merzenich, M.M. (1996). Language comprehension in languagelearning impaired children improved with acoustically modified speech. Science 271: 81-84.

Tremblay, K., Kraus, N., McGee, T., Ponton, C. and B. Otis (2001). Central auditory plasticity: changes in the N1-P2 complex after speech-sound training. Ear Hea. 22: 79-90.

van Praag, H., Kempermann, G. and Gage, F.H. (2000). Neural consequences of environmental enrichment. Nat Rev Neurosci 1: 191-198.

Volkmar, F.R. and Greenough, W.T. (1972). Rearing complexity affects branching of dendrites in the visual cortex of the rat. Science 176(42): 1145-7.

Warrier, C.M., Johnson, K.L., Hayes, E.A., Nicol, T.G. and Kraus, N. (2004). Learning impaired children exhibit timing deficits and training-related improvements in auditory cortical responses to speech in noise. Exp Brain Res 157:431-41. 


\section{VITA}

Cherie Renee Perroncel was born July 11, 1978 in Lafayette, Louisiana to Colleen Frances and Douglas Wade Perroncel. March 1, 1987, Cherie was hit by a car in Springfield, Illinois, and was care-flighted to Dallas. Requiring CPR and brain surgery, she would spend 4 weeks in a coma and several months in the hospital. The doctors told her mom that if Cherie lived through the night she would never be more than a "vegetable". After months of intensive speech, occupational, and physical therapy, Cherie made a complete recovery. In 1996, she graduated in the top 10 from Forney High School with an advanced honors diploma. Cherie went on to excel at Texas A\&M University in College Station, finishing in only 3 years, with a Bachelor of Science degree in Psychology. At A\&M, she earned the Distinguished Student Award 1998-1999. In 1999, Cherie entered graduate school at The University of Texas Dallas, and in 2001 completed her first Master of Science degree in Human Development and Early Childhood Disorders. She was originally trained as a clinician, specializing in neurodevelopmental therapy for children with autism, under the guidance of Michelle MacAlpine. In 2001, Cherie entered the doctoral program in Neuroscience under the supervision of Mike Kilgard, and a year later, earned her second Master's degree in Applied Cognition and Neuroscience. She will complete post-doctoral studies at The University of Texas in Austin under the direction of Theresa Jones. 